\title{
ADVANCES IN PARTICLES AND FIELD RESEARCH IN THE SATELLITE ERA
}

\section{GPO PRICE}

CFSTI PRICE(S) \$

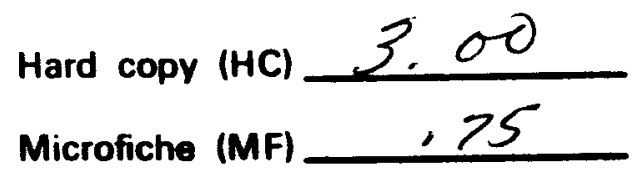

BY

W. HESS

G. MEAD

ff 853 July 65

M. P. NAKADA

$\begin{array}{r}0 \\ 0\end{array} \mid$
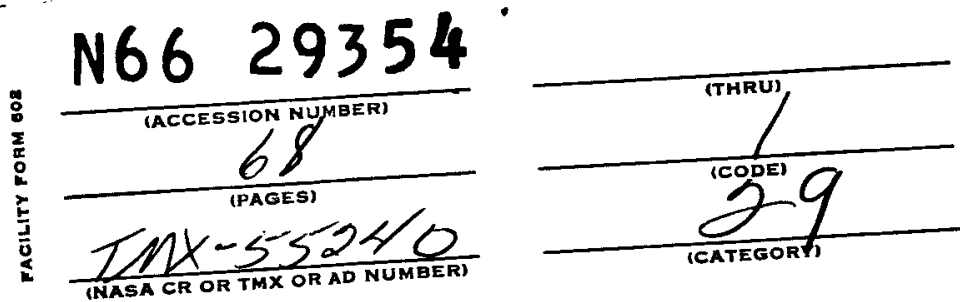

\section{JANUARY 1965}

\section{NASA}

GODDARD SPACE FLIGHT CENTER GREENBELT, MARYLAND

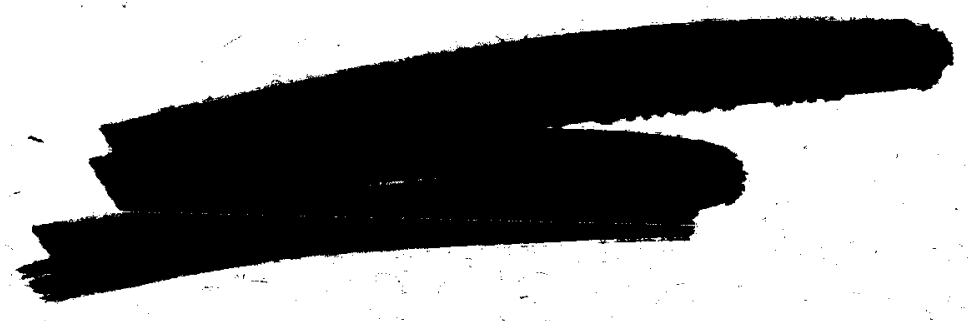




\title{
ADVANCES IN PARTICLES AND \\ FIELD RESEARCH IN THE \\ SATELLITE ERA
}

\author{
by \\ W. Hess, G. Mead and M. P. Nakada \\ Theoretical Division \\ NASA-Goddard Space Flight Center \\ Greenbelt, Maryland
}

January 1965 


\section{PREFACE}

The Space Age started in 1957 at a time when a number of scientific investigations in geophysics had matured considerably and were progressing only slowly. The measurements possible with traditional methods were too incomplete to lead to a theoretical understanding of many phenomena. Specifically, one had to postulate the existence of solar radiations which could not be observed from the ground. These were required to explain observations in such diverse fieids as geomagnetism, auroral studies, cosmic rays, comets, and even of long time weather trends. The possibility of conducting continuous measurements from above the earth's atmosphere or even from outside the terrestrial magnetic field has generated renewed interest in geophysics and is contributing greatly to our understanding of many new aspects of the earth's environment.

The contributions of space science can be appreciated properly only if placed into the historical context of the pertinent scientific fields. Records of the observations of Northern lights or aurorae go back 2,000 years. Similarly, the discovery of the earth's magnetic field cannot be dated with certainty but its existence was already known by the year 1200 A.D. By $1722 \mathrm{Grahm}$ had discovered rapid fluctuations in the geomagnetic field; these were correlated with the existence of auroral displays in 1741 by Hiorter, and further correlated with the sunspot cycle by Sabin in 1852.

The number and quality of crround based observations continued to increase during this century and culminated in the International Geophysical Year of 1958. Various theoretical explanations were advanced by different investicators; out standing among these is the work of Chapman and Ferraro. They postulated that streams of energetic plasma coming from the sun interact with the earth's magnetic field to produce magnetic field fluctuations. It was presumed that these are also responsible in some way for the visual auroral display. Evidence for the existence of solar plasma streams came independently from Biermann's analysis of the behavior of the tails of comets and Parker showed in 1958 , on theoretical grounds, that such a plasma flow could be produced by the free expansion of the solar corona.

The energetic plasma stream from the sun, senerally called the solar wind, has by now been observed with space probes and satellites, and it detailed properties and time fluctuations are under intensive investigation. Similarly, we are observing the interaction of the solar wind with the earth's magnetic field and are studying the way in which it causes aurorae. Naturally, it has not been possible to solve in the few years of the Space Age all the problems which have puzzled scientists for centuries, but the key to the understanding of this aspect of nature is now within our reach.

Historically an entirely different field of investigation has been the study of cosmic rays. They were discovered in the early 20 th century and have been investigated since then with detectors placed at mountain locations or in balloons. Most of the observations had to be confined to the energetic component of the cosmic rays, greater than $1 \mathrm{Bev}$, because only these were able to penetrate through the earth's magnetic field and reach balloon altitudes. Forbush had found a rather surprising modulation of the cosmic ray flux by solar activity. He found a general decrease of this flux during periods of maximum activity of the sun, which follows an 11 -year cycle. Further, he found short term decreases after larger solar flares, which recover in a few days. 
Space probe and satellite observations have produced direct measurements of the mechanism by which the sun modulates the intensity of galactic cosmic rays. The plasma of the solar wind carries with it an imbedded magnetic field and the strength and direction of this field is affected by the frequency and size of the solar flares. Although the strength of this field is only $1 / 10,000$ of the magnetic field at the surface of the earth, it is effective in preventing even very high energy particles from penetrating the solar system to the earth. The distances involved are large enough (several times 100,000,000 miles) that minute changes in direction accumulate to deflect particles with energies of many thousands of Bev.

Space measurements are required to investigate the low energy end (below $100 \mathrm{Mev}$ ) of the cosmic ray spectrum, because these observations can be made properly only from outside the geomagnetic field. Of particular interest in this energy region are the solar cosmic rays which follow some solar flares. Although these were discovered before the Space Age, only their general character could be investigated until rocket and satellite observations became possible. An understanding of solar proton events is of immediate practical importance because they constitute a radiation hazard to biological organisms.

Space flight has not only provided the means for finding solutions to problems previously discovered in other fields of scientific inquiry but has also led to discoveries of entirely new and unexpected phenomena. Outstanding among these was the discovery by Van Allen of the radiation belts. Further investigations have shown that a region exists extending from above the atmosphere out to roughly 35,000 miles in which energetic protons and electrons can be trapped by the earth's magnetic field. This region has been divided into so-called "belts" depending on the character of the dominant particles; of these, the inner proton belt, centered around an altitude of 1,500 miles, was discovered first and consists of penetrating protons (energies above $30 \mathrm{Mev}$ ). The radiation belts pose a problem to civilian and military manned space flight because of the radiation hazard from both natural and artificial sources, i.e., nuclear tests. For this reason an understanding of trapping mechanisms and lifetimes of energetic particles is also of practical importance.

It emerges, therefore, that the Space Age started at a most opportune time to contribute to human knowledge. Not only had scientists arrived at a stage where space measurements were required to confirm or disprove their theories, but conversely new discoveries like the Van Allen belts could be interpreted in a meaningful way on the basis of physical laws already developed in other research. As space research progresses, a comprehensive understanding is emerging of the earth-sun relations and of the solar system. This knowledge in turn will lead to new insight into the structure of the universe and permit the evolution of more realistic cosmological models. Beyond the scientific aspects of space research are the practical applications. The hazard posed by energetic particle radiations has already been mentioned. Energetic particles are also being precipitated into the atmosphere and have a pronounced effect on the ionosphere and upper atmosphere. The full implications of these effects have yet to be understood; however, the influence on radio communication is well established and is under intensive investigation.

A survey of the new findings and accomplishments is given in the paper which follows. It may be appropriate, however, to consider at this point the contributions made by the different participants in the particles and fields area. Both the U.S. and the USSR have launched numerous satellites and space probes. The USSR program was aimed more toward feats such as first manned space flight or first pictures of the back side of the moon than towards a systematic investigation of the space environment. Their observations in this area have been confined to relatively low altitudes with only isolated observations from space probes such as Lunik II; and thus, their data by themselves cannot be interpreted uniquely. On the other hand, NASA has launched satellites into highly 
eccentric orbits that go out beyond 60,000 miles; these satellites were equipped with the specialized magnetometers and radiation detectors which are required for this work. Thus, essentially all of the recent scientific results on the solar wind, interplanetary magnetic fields, the magnetosphere, the outer radiation belts, and cosmic ray energy spectra below $100 \mathrm{Mev}$ have resulted from the NASA program. In the exploration of the inner Van Allen belts both the NASA and Department of Defense Programs have made substantial contributions. An example of the cooperation which has existed in this field was the exploration of the artificial radiation belt produced by the July 9, 1962 nuclear test. The NASA program was concentrated on equatorial measurements of the belt while the DOD observations were conducted with polar orbiting spacecraft which measured primarily those electrons with low mirror points. In the area of planetary investigations, Mariner II, launched by NASA, has furnished the only observations to date which were conducted near another planet.

A. W. Schardt Physics and Astronomy Programs NASA Headquarters 


\section{CONTENTS}

Page

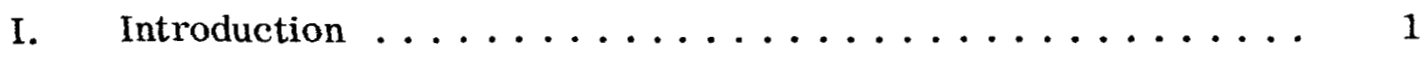

II. The Solar Wind and Its Effect on the Earth ........... 5

A. The Solar Wind and Interplanetary Field ......... 5

B. The Magnetosphere $\ldots \ldots \ldots \ldots \ldots \ldots$

C. The Transition Zone and Shock Wave............ 14

III. Radiation Belts ...................... 21

A. Discovery of the Belts .................. 21

B. The Inner Radiation Belt .................. 23

C. Artificial Radiation Belts ................ 26

D. The Outer Radiation Belt ................ 29

E. Other Belts..................... 33

F. The Aurora $\ldots \ldots \ldots \ldots \ldots \ldots \ldots \ldots \ldots \ldots$

IV. The Earth's Magnetic Field ................ 39

V. Solar Energetic Particles and Ground Effects . . . . . . . 43

VI. Cosmic Rays ...................... 49

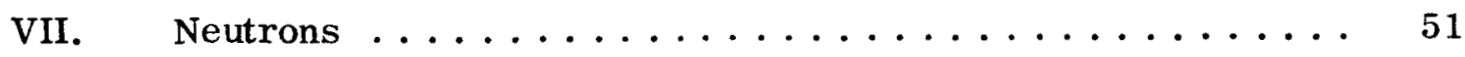

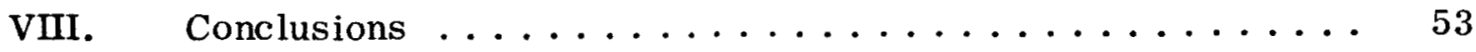




\section{LIST OF ILLUSTRATIONS}

Figure

Page

1 Orbits of various eccentric satellites and space probes projected into the equatorial plane. ............

2 The spiral form of the interplanetary magnetic field lines caused by the radial outflow of solar wind and rotation

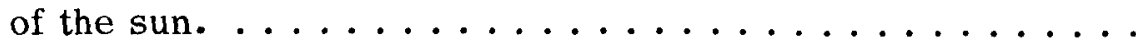

3 Typical energy spectra of the solar wind as measured on Mariner II. The second peak sometimes present is very likely due to the presence of the He nuclei along with

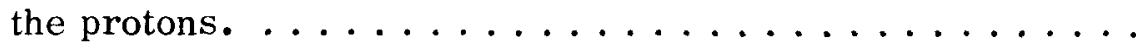

4 Magnetic field data from Explorer XII showing an abrupt change in the field at $8.2 \mathrm{R}_{\mathrm{e}}$. This change, the magnetopause, is the outer limit of the geomagnetic field. ...........

5 Theoretical shapes of the intersection of the magnetopause with planes parallel to the earth-sun line. ...........

6 Theoretical shapes of distorted geomagnetic field lines in the magnetosphere noon-midnight meridian plane. The dotted lines are dipole field lines shown for comparison. ......

7 A theoretical model of the tail of the magnetosphere. Friction at the magnetopause pulls the field lines back and produces a

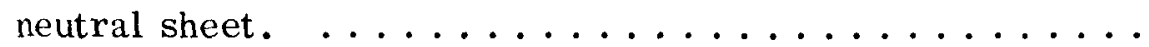

8 Magnetic field data from orbit 11 of Explorer XVIII (IMP-1). The magnetopause is at $13.6 \mathrm{R}_{e}$. The second transition at $20 \mathrm{R}_{\mathrm{e}}$ to an ordered field outside is the location of the bow

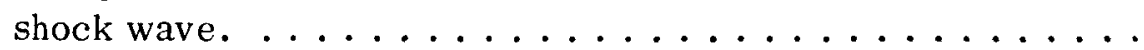

9 A sphere in a supersonic wave tunnel showing a detached shock wave upstream from the object. .............

Solar wind data from the MIT plasma probe on IMP-1. In the interplanetary medium the detector shows large fluxes in only one energy channel. In the transition zone there are significant fluxes in all channels (including the electron

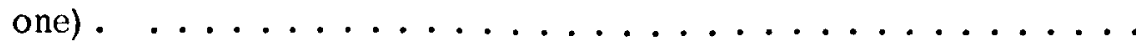


11 Solar wind data from the Ames plasma detector on IMP-1. The inner boundary of the wind at $11 \mathrm{R}_{\mathrm{e}}$ is approximately at the magnetopause. The sharp transition at $16 \mathrm{R}_{\mathrm{e}}$ is the bow shock wave location. In the transition region protons of all energies are present. In the interplanetary medium protons are only present in one channel. ..........

12 Flux of $\mathrm{E}>40 \mathrm{Kev}$ electrons detected on IMP. The radiation belt is present inside the magnetopause at $10 R_{e}$, and outside this occasional "islands" of particles are observed. . .

13 The location of the magnetopause and bow shock as determined by the magnetometer on IMP-1. Shown for comparison are the theoretically expected locations. ...........

14 On December 14 and 15 the interplanetary magnetic field measured by IMP-1 was unusually disturbed. This was the period of closest eclipse by the moon and may indicate a magnetic tail behind the moon as shown here. ............

15 Van Allen's picture of the inner and outer zone of the radiation belt made after Pioneer III. ...............

16 Energy spectrum of protons in the inner radiation zone as measured by nuclear emulsions. ............

17 A map showing the spatial distribution of energetic protons ( $\mathrm{E}>30 \mathrm{Mev}$ ) in the inner zone of the radiation belt. This map uses McIlwain's B-L coordinate system. ........

18 The proton energy spectrum at different locations in the inner zone showing the lower energy protons present in the outer

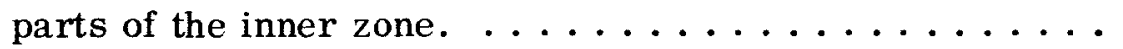

Spatial distribution of high-energy protons measured by McIlwain on Explorer XV, showing the double-peaked distribution. (This is an R- $\lambda$ magnetic coordinate map as obtained from transformation of B-L coordinates, and is not the same as a geographic map). ................. 
20 Energy spectra of electrons in the Starfish artificial radiation belt as measured on satellite $1962 \beta \mathrm{k} . \ldots \ldots \ldots \ldots$

21 Decay of the Starfish artificial radiation belt electrons at low altitudes as measured on Injun. . . . . . . . . . . 28

22 Rapid decay of the Starfish electrons at high altitudes as measured on Telstar. ................... 29

23 Energy spectra of low-energy protons measured by Explorer XII at several locations in the outer zone of the radiation belt. . . 31

24 The dotted lines are theoretical curves showing how the e-folding energy $\mathrm{E}_{\mathrm{O}}$ should vary assuming the outer zone protons move radially, conserving $\mu$ and $I$. The solid lines are the experimental values of $E_{O}$ from Explorer XII data. .........

25 Time variations of $\mathrm{E}>5 \mathrm{Mev}$ electrons in the outer zone measured on Explorer XV. .................

26 Flux contours of $\mathrm{E}>40 \mathrm{Kev}$ electrons in the outer radiation belt showing the day-night asymmetry. . . . . . . . . . 34

27 Simultaneous observation of trapped electrons dumped electrons and auroral light emission. These and other studies indicate an increase in trapped radiation when auroras occur. . . . 3 36

28 Sample of precipitated flux. Note that near $\mathrm{L}=6$ some precipitated flux is observed at all times since all observations show more than background fluxes. ........... 36

29 A test of diffusion theory applied to solar protons. After about one hour the test works. ................. 45

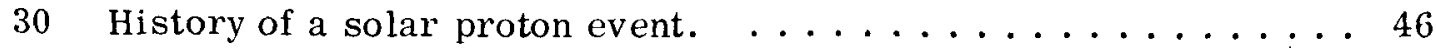

31 A recurrent solar proton event measured on Explorer XII in October, 1961. .................. 47

32 Proton energy spectra from several solar proton events. . . . 47 


\title{
ADVANCES IN PARTICLES AND FIELD RESEARCH
}

\author{
IN THE SATELLITE ERA \\ by \\ W. Hess, G. Mead and M. P. Nakada \\ Theoretical Division \\ NASA-Goddard Space Flight Center \\ Greenbelt, Maryland
}

I.

\section{INTRODUCTION}

Before the satellite era we had relatively little knowledge of the environment of the earth above $100 \mathrm{~km}$. The existence of the ionosphere was known originally from a study of radio waves and later from direct observations by balloons and rockets. Measurements on the zodiacal light had indicated that there were electrons through all space near the sun, but how many and moving in what fashion was quite uncertain. From a knowledge of the earth's surface magnetic field one could predict the field some distance into space. From this data a crude picture of near-earth space had developed which we now know to be very incomplete and rather wrong. The first U.S. satellite started in a dramatic way to revise our picture of the environment.

The first indication of the existence of geomagnetically-trapped radiation came early in 1958 with the launch of Explorers I and III. Van Allen observed an anomalously high counting rate, followed by a period of zero count rate due to saturation of the geiger counter as the satellite entered into the radiation belt. These first experiments have been followed by innumerable others which have confirmed the existence of two broad zones of penetrating particles-an inner zone primarily consisting of protons and an outer zone predominantly electrons. The composition, energy spectra and temporal and spatial variation of these belts have been extensively studied by satellites and space probes. In addition, a series of U.S. and Russian nuclear explosions in space have demonstrated the possibility of producing long-lived artificial radiation belts.

As the United States developed the capability of launching satellites into highly eccentric orbits (see Table I and Figure 1) a new phenomenon was discovered: the existence of an outer boundary to the earth's magnetic field and 
Table 1

DATA ON IMPORTANT SATELLITES AND SPACE PROBES IN PARTICLES AND FIELDS RESEARCH

\begin{tabular}{|c|c|c|c|c|c|c|c|}
\hline Satellite & Active Life & $\underset{\mathrm{Km}}{\text { Apogee }}$ & $\left|\begin{array}{c}\text { Perigee } \\
\mathrm{Km}\end{array}\right|$ & $\begin{array}{l}\text { Inclina- } \\
\text { tion }\end{array}$ & Periva $[$ S & \begin{tabular}{|} 
Sun-Earth-Apogee \\
Angle Projected on \\
Equatorial Plane
\end{tabular} & Types of Apparatus \\
\hline Spurnik II & $\begin{array}{l}\text { Nov. 3, } 1957 \\
\text { to } \\
\text { Nov. } 9,1957\end{array}$ & 1670 & 225 & $65^{\circ}$ & $104 \mathrm{~min}$ & & Geiger Counters \\
\hline Explorer I & $\begin{array}{l}\text { Jan. 31, } 1958 \\
\text { to } \\
\text { March 15, } 1958\end{array}$ & 2500 & 360 & $33^{\circ}$ & $115 \mathrm{~min}$ & & Geiger Counter \\
\hline Sputnik III & $\begin{array}{c}\text { May } 15,1958 \\
\text { to } \\
\text { June } 17,1958\end{array}$ & 1880 & 226 & $65^{\circ}$ & $90 \mathrm{~min}$ & & Scintillator \\
\hline Explorer IV & $\begin{array}{l}\text { July } 26,1958 \\
\text { to } \\
\text { Sept. } 19,1958 \\
\end{array}$ & 2200 & 260 & $51^{\circ}$ & $110 \mathrm{~min}$ & $-\begin{array}{lll}- & \ldots\end{array}$ & Geiger Counters and Scintillotors \\
\hline Explorer VI & $\begin{array}{l}\text { Aug. } 7,1959 \\
\text { to } \\
\text { Oct. } 6,1959\end{array}$ & 42,000 & 230 & $50^{\circ}$ & $12.7 \mathrm{~min}$ & $140^{\circ}$ to $70^{\circ}$ & Energetic Particle Detectors and Magnetometers \\
\hline Vanguard III & $\begin{array}{l}\text { Sept. } 18,1959 \\
\text { to } \\
\text { Dec. } 12,1959\end{array}$ & 3730 & 500 & $33^{\circ}$ & $130 \mathrm{~min}$ & - & Mognetometer \\
\hline Explorer VII & $\begin{array}{l}\text { Oet. } 13,1959 \\
\text { to } \\
\text { Feb. } 1961\end{array}$ & 1090 & 555 & $50^{\circ}$ & $101 \mathrm{~min}$ & $\cdots-$ & Geiger Counters \\
\hline Sputnik V & $\begin{array}{l}\text { Aug. } 19,1960 \\
\text { to } \\
\text { Aug. } 20,1960\end{array}$ & 300 & 300 & $65^{\circ}$ & $91 \mathrm{~min}$ & $\cdots-\cdots$ & Geiger Counters and Scintillators \\
\hline Explorer $X$ & $\begin{array}{c}\text { Morch 25, } 1961 \\
\text { to } \\
\text { Morch 27, 1961 }\end{array}$ & 290,000 & 160 & $33^{\circ}$ & $112 \mathrm{hrs}$ & $130^{\circ}$ & Plasma Probe and Magnetometer \\
\hline Iniun I & $\begin{array}{c}\text { June } 29,1961 \\
\text { to } \\
\text { April } 1963\end{array}$ & 1020 & 860 & $67^{\circ}$ & $104 \mathrm{~min}$ & - & $\begin{array}{l}\text { Energetic Particle Detectors, CdS Detector, } \\
\text { and Magnetometer }\end{array}$ \\
\hline Explorer XII & $\begin{array}{c}\text { Aug. } 16,1961 \\
\text { to } \\
\text { Dec. } 6,1961\end{array}$ & 77,000 & 300 & $34^{\circ}$ & $26.5 \mathrm{hrs}$ & $360^{\circ}$ to $260^{\circ}$ & $\begin{array}{l}\text { Energetic Particle Detectors, Cosmic Ray } \\
\text { Detector, Plasma Probe and Magnetometer }\end{array}$ \\
\hline TRAAC & $\begin{array}{c}\text { Nov. } 15,1961 \\
\text { to } \\
\text { Aug. } 14,1962\end{array}$ & 1160 & 900 & $32^{\circ}$ & $106 \mathrm{~min}$ & & Energetic Particle Detectors \\
\hline Cosmos III & $\begin{array}{l}\text { April } 24,1962 \\
\sim 1 \text { yeor }\end{array}$ & 670 & 210 & $49^{\circ}$ & $92 \min$ & $-\quad-\quad-\quad-$ & $\begin{array}{l}\text { Geiger Counter Scintillotor and } \\
\text { Magnetometer }\end{array}$ \\
\hline Telstor I & July 10,1982 & 5700 & 950 & $45^{\circ}$ & $158 \mathrm{~min}$ & - & Solid State Detectors \\
\hline Explorer XIV & $\begin{array}{l}\text { Oct. } 2,1962 \\
\text { to } \\
\text { Feb., } 1964\end{array}$ & 100,000 & 280 & $33^{\circ}$ & $36.4 \mathrm{hrs}$ & & $\begin{array}{l}\text { Energetic Porticle Detector, Cosmic Ray } \\
\text { Detector, Plasma Probe, and } \\
\text { Magnetometer }\end{array}$ \\
\hline Explorer XV & $\begin{array}{l}\text { Oct. } 27,1962 \\
\text { to } \\
\text { Feb. } 3,1963\end{array}$ & 17,300 & 310 & $18^{\circ}$ & $5.2 \mathrm{hrs}$ & -.. & $\begin{array}{l}\text { Energetic Particle Detectors and } \\
\text { Magnetometer }\end{array}$ \\
\hline Relay I & Dec. 13,1962 & 7400 & 1310 & $47^{\circ}$ & $185 \mathrm{~min}$ & & Energetic Particle Detectors \\
\hline $\begin{array}{l}\text { Explorer XVIII } \\
\text { (IMP I) }\end{array}$ & $\begin{array}{l}\text { Nov. } 26,1963 \\
\text { to }\end{array}$ & 200,000 & 200 & $33^{\circ}$ & 3.9 doys & $335^{\circ}$ to $160^{\circ}$ & $\begin{array}{l}\text { Energetic Particle Detectors, Cosmic Ray } \\
\text { Detector, Plasmo Probe, and Magnetometer }\end{array}$ \\
\hline EGO & Sept. 5, 1964 & 149,000 & 280 & $33^{\circ}$ & 64 hrs & 140 to & $\begin{array}{l}\text { Energetic Porticle Detectors, Cosmic Roy } \\
\text { Detectors Plasma Probes, and Magnetometers }\end{array}$ \\
\hline Probes & Launch Date & \multicolumn{4}{|c|}{ Orbit } & $\begin{array}{l}\text { Apogee-Earth- } \\
\text { Sun Angle }\end{array}$ & Types of Apparatus \\
\hline Pioneer III & Dec. 6,1958 & \multicolumn{4}{|c|}{$109,000 \mathrm{~km}$ maximum altitude } & $340^{\circ}$ & Geiger Counters \\
\hline Pioneer IV & March 3, 1959 & \multicolumn{4}{|c|}{ heliocentric orbit } & $320^{\circ}$ & Geiger Counters \\
\hline Lunik II & Sept. 12, 1959 & \multirow{2}{*}{\multicolumn{3}{|c|}{$\begin{array}{l}\text { imported on moon } \\
\text { heliocentric orbit }\end{array}$}} & & \multirow[t]{2}{*}{$140^{\circ}$} & Like Lunik I \\
\hline Moriner II & Aug. 27, 1962 & & & & & & $\begin{array}{l}\text { Energetic Particle Detectors, Plasma Probe, and } \\
\text { Mognetometer }\end{array}$ \\
\hline
\end{tabular}




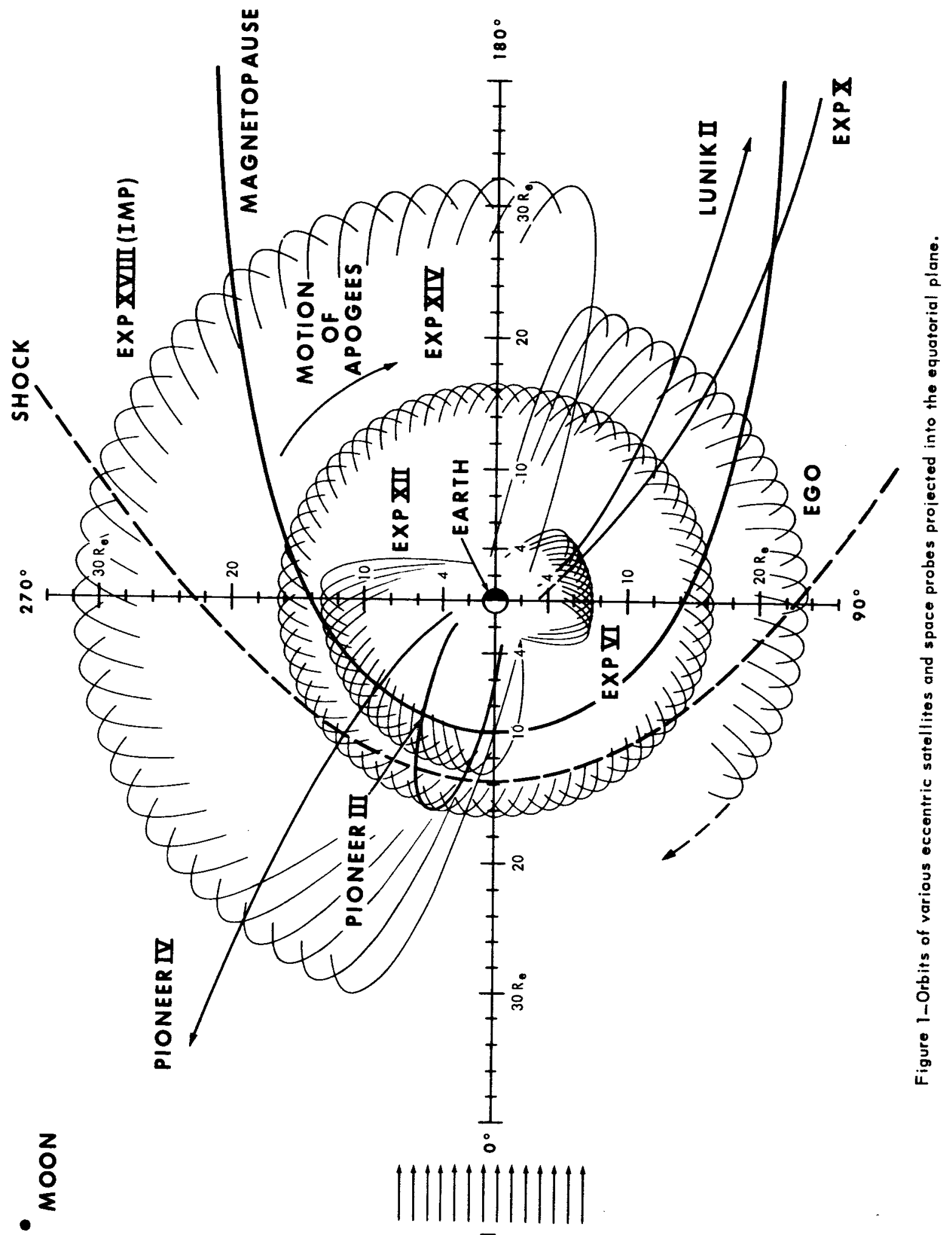

总 
the trapping region. This boundary, termed the magnetopause or outer edge of the magnetosphere, was located at about 10 earth radii from the center of the earth in the solar direction. The boundary of the trapping region is closer in on the night side, but the magnetic boundary extends to much larger distances. U.S. and Russian space probes and satellites have made direct observations of the solar plasma responsible for the existence of the boundary. The term solar wind has been applied to emphasize that it is present at all times.

Much of the electromagnetic radiation and most of the particles which are emitted from the sun, particularly during disturbed times, never reach the earth's surface because of the protection of the earth's atmosphere and magnetic field. The same is true of much of the cosmic radiation. Satellites and space probes, particularly those which pass outside the magnetopause, have measured this radiation directly and have thereby greatly increased our understanding of solar and cosmic ray physics. Of special interest here are the occasional emissions by the sun of streams of energetic protons in connection with certain solar flares.

We have attempted in this document to summarize the advances that have been made in particles and fields research in the satellite era and have concentrated on those subjects about which our understanding has increased most dramatically since 1958 . 
II.

THE SOLAR WIND AND ITS EFFECT ON THE EARTH

A. The Solar Wind and Interplanetary Field

Before the satellite era, Biermann had conjectured that the explanation of comet tails pointing away from the sun required more than just light pressure, and that it was very likely that energetic solar plasma accompanied by magnetic fields was continually present.(1) Parker demonstrated theoretically that the solar corona was unstable and must be expanding continuously. ${ }^{(2,3)}$ He studied the hydrodynamic expansion of the solar corona with a simple spherically symmetric model and was able to deduce plasma velocities and densities from coronal properties. His studies indicated that a continuous wind should exist. He also estimated the strength and direction of the interplanetary field under the assumption that this field was of solar origin and was carried along by the solar wind. Due to the rotation of the sun the solar field lines should have the form of an Archimedes spiral. (See Figure 2.)

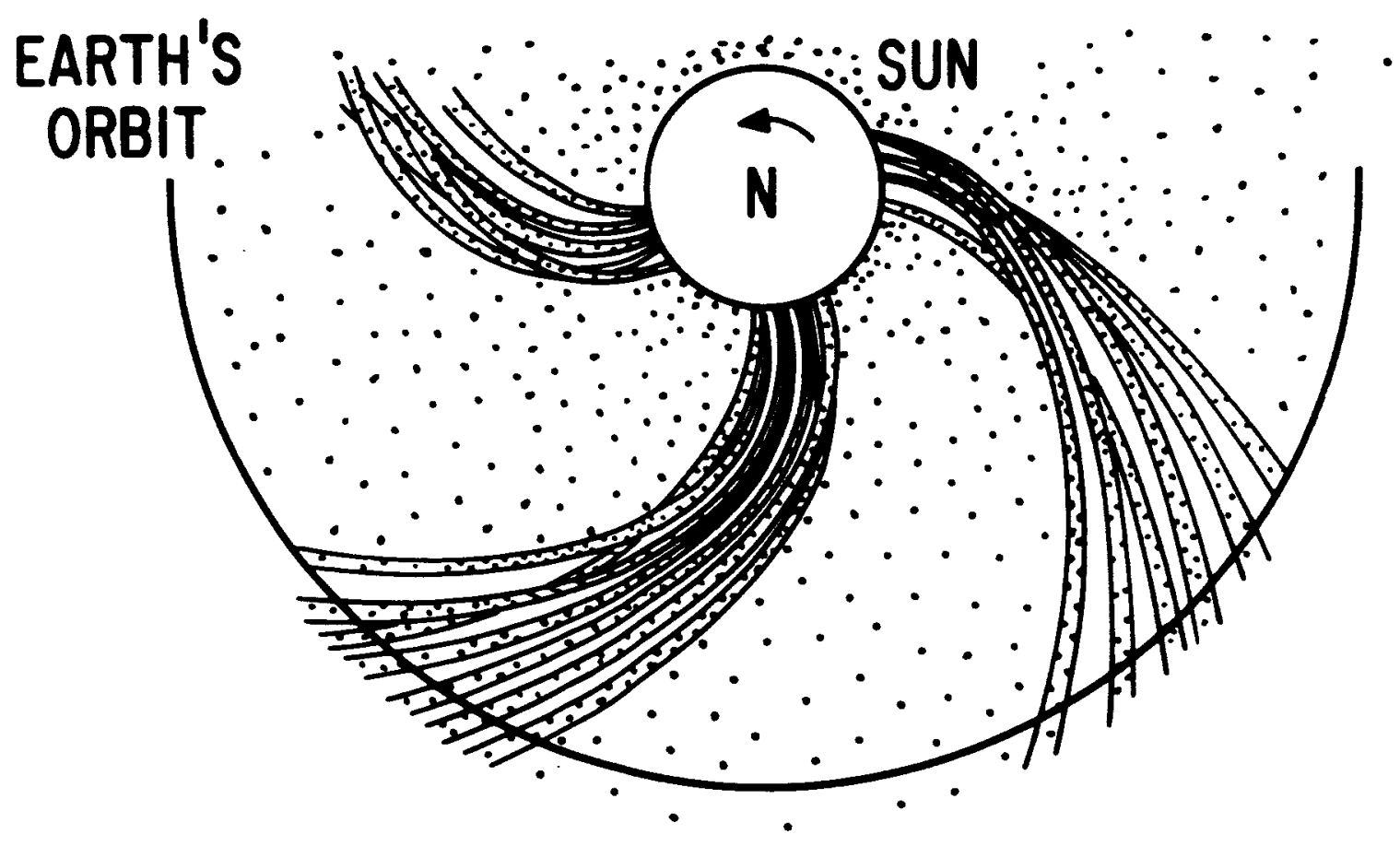

Figure 2-The spiral form of the interplanetary magnetic field lines caused by the radial outflow of solar wind and rotation of the sun. 
Interplanetary space probes have given striking confirmation to the ideas and calculations of Biermann and Parker.

Gringauz on Lunik 2 and 3 measured interplanetary plasma fluxes, ${ }^{(4)}$ but did not measure particle energies and therefore could not differentiate between a light breeze and a solar wind, nor determine the direction of flow. They found fluxes of about $10^{8} \mathrm{part} / \mathrm{cm}^{2}-\mathrm{sec}$.

A Faraday cup flown on Explorer X by Bridge and others at MIT confirmed the Lunik fluxes and also indicated a definite wind that came approximately from the sun with a velocity of about $300 \mathrm{~km} / \mathrm{sec}$. ${ }^{(5)}$

More recent measurements by Neugebauer and Snyder ${ }^{(6,7,8)}$ on Mariner II and the MIT $^{(9)}$ and Ames ${ }^{(10)}$ groups on Explorer 18 (IMP-1) have extended over long enough times to indicate that

a. a definite wind ( $\sim 300-500 \mathrm{~km} / \mathrm{sec})$ blows at all times;

b. the energy spread in the wind is narrow compared to the average directed energy $(\triangle E / E \sim .01)$;

c. the wind comes nearly radially from the sun;

d. protons and He nuclei appear to be present (see Figure 3);

e. the wind is very gusty-showing fluctuations in energy, energy spread, and density in times of the order of hours.

Longer term studies with Mariner II results show a striking correlation between geomagnetic activity and daily average solar wind velocity. ${ }^{(8)}$ The correlation between solar wind velocity and the 27-day solar rotation as well as the velocity fluctuations seem to indicate that the wind characteristics depend more on local conditions in the solar corona rather than on over-all solar properties.

Even before the solar wind properties were determined, the interplanetary magnetic field was measured by Sonett et al. on Pioneer V. (11) The field component perpendicular to the vehicle axis was measured by a search coil and found to be about $3 \%$. At the time of a large disturbance the field went up to $40 \%$. More precise and long term measurements have been made by Mariner $\mathrm{II}^{(12)}$ and IMP-1. ${ }^{(13)}$ This interplanetary field has an energy density much smaller $(\sim .01)$ than solar wind energy densities, so it is carried along by the solar wind. Field values are usually between 2 and 7 gammas and average near 5 gammas. The 


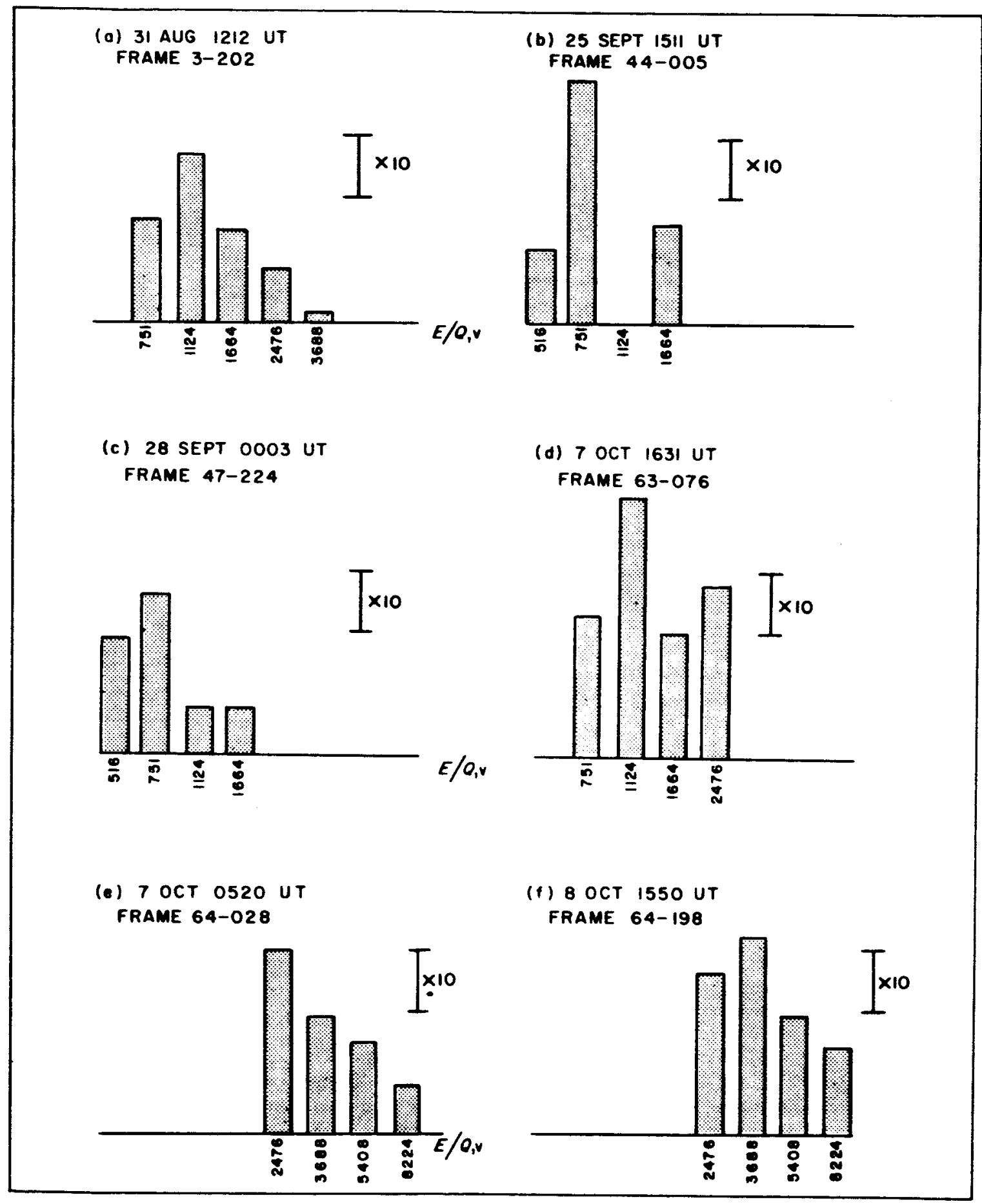

Figure 3-Typical energy spectra of the solar wind as measured on Moriner II. The second peak some. times present is very likely due to the presence of the He nuclei along with the protons. 
magnitude of the field has been found to be relatively constant compared to changes in the direction of the field. Although the field direction does not always fit the simple picture of an Archimedian spiral as predicted by Parker, average values have a tendency to lie along such a spiral.

Ness has found from Explorer 18 (Imp I) data that the field has a filamentary structure showing reversals in direction with nulls in between filaments. ${ }^{(13)}$

Field measurements on Pioneer I showed a turbulent disturbed field in the region of roughly 50,000 to $100,000 \mathrm{~km} .{ }^{(14)}$ We now know this is the transition zone inside the bow shock wave. Sonett studied the form of the magnetic shock waves found in this region. ${ }^{(15)}$

A collisionless shock wave was also found in interplanetary space by Mariner. ${ }^{(16)}$ Shock waves should result from high speed solar wind plowing into slower moving wind. This is discussed more in section IIC.

Thus far space probes have sampled interplanetary space only at about 1 A.U. and close to the ecliptic plane. Our ideas of the solar wind say that the velocity should be independent of distance from the sun over some considerable range of distances and that the density should fall off as $1 / \mathrm{r}^{2}$. Measurements of Snyder and Neugebauer on Mariner II do indicate a $1 / \mathrm{r}^{2}$ fall-off fairly well.

We can use information concerning comet tails to aid in understanding the solar wind. What is known about type 1 comet tails indicates that the solar wind is present all the time and is not confined to the ecliptic plane, but extends as well to high solar latitudes although with possibly a smaller flux than near the ecliptic plane.

Using the measured solar wind fluxes near the earth and assuming isotropic emission from the sun, we can estimate the loss of mass from the sun due to the solar wind outflow. This rate is approximately $10^{-5}$ of the solar mass in $10^{9}$ years. At this rate the total mass loss due to solar wind would be insignificant in the estimated lifetime of the sun. This loss rate is about 0.1 of the solar mass lost by thermonuclear reactions.

\section{B. The Magnetosphere}

It has long been realized that plasmas and magnetic fields tend to confine one another. In an experimental machine such as a stellerator, for example, a strong magnetic field can compress and confine a hot dense plasma within a small region of space, without the need for confining walls. In like fashion, if a streaming plasma encounters a magnetic object such as a magnetized sphere, 
the plasma will confine the magnetic field to a limited region about the object. The object, in turn, will tend to exclude the plasma, creating a hole or cavity. The size of the cavity is determined by the energy density of the streaming plasma and the degree of magnetization of the object.

In addition, if the velocity of the plasma is sufficiently great as to be highly supersonic in the magnetohydrodynamic sense-that is, if the velocity is much higher than the Alfvén velocity in that medium-a detached shock wave may be produced in the region ahead of the cavity boundary. This process is analogous to the formation of the detached shock wave in front of an aerodynamic object traveling at hypersonic speeds through the atmosphere (Mach numbers greater than 5).

In 1931 Chapman and Ferraro first predicted the confinement of the earth's magnetic field inside an elongated cavity during magnetic storms. ${ }^{(17)}$ The continual presence of such a cavity has been experimentally verified by a great many satellites observations including those made by Explorers 10, 12, 14, and 18 (IMP's-1) and 21 (IMP-2). Figure 4 shows one radial pass of Explorer 12.

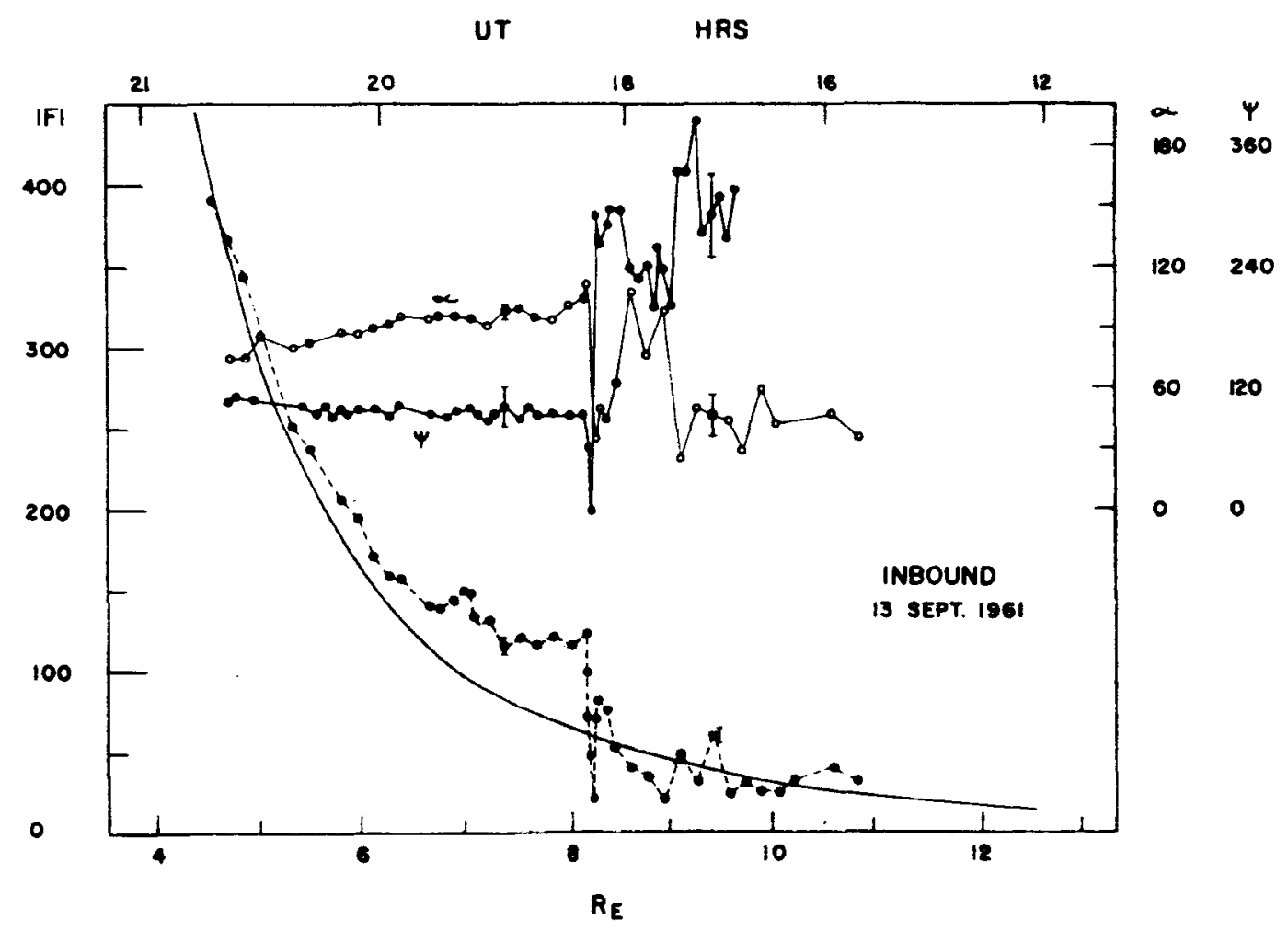

Figure 4-Magnetic field data from Explorer XII showing an abrupt change in the field at $8.2 R_{e}$. This change, the magnetopause, is the outer limit of the geomagnetic field. 
Cahill's magnetometer record ${ }^{(18)}$ shows the expected radial decrease until at $8.2 R_{e}$ the field suddenly changes magnitude and starts wandering in direction and strength. At this same radial location the trapped radiation belt flux suddenly fell essentially to zero. The region inside the cavity ${ }^{(19)}$ is called the magnetosphere, and the boundary is termed the magnetopause. In addition, Explorer 18 (IMP-1) has verified the presence of a detached shock wave.(13) The region between the magnetopause and the shock wave is usually referred to as the transition region. Outside this transition region, i.e., beyond the shock wave, conditions are characteristic of the interplanetary medium, and the presence of the magnetized earth has little or no effect.

The dimensions of the cavity depend, of course, on the intensity of the solar wind, although the dependence is rather weak. That is, large changes in the solar wind intensity produce comparatively small changes in the size of the cavity. The distance from the center of the earth to the magnetopause in the solar direction is typically around 10 earth radii, although distances less than $8 R_{e}$ and more than $13 R_{e}$ have occasionally been observed. The shock wave is located several earth radii beyond this. At $90^{\circ}$ to the solar direction, both the magnetopause and shock wave are observed to flare out to distances about 30-50 percent greater than the subsolar distances. In the anti-solar direction the cavity extends out to very large distances, very likely as far as the moon or further, i.e., $60 \mathrm{R}_{\mathrm{c}}$. No closure of the magnetosphere tail has yet been observed by satellites.

Much progress has been made recently in our theoretical understanding of the way in which a streaming plasma such as the solar wind interacts with a dipole field. One of the basic problems has been to determine the shape of the boundary (i.e., the magnetopause). Beard ${ }^{(20)}$ Midgley and Davis, ${ }^{(21)}$ and Mead and Beard ${ }^{(22)}$ have obtained numerical results for the shape of the boundary between a field-free, zero-temperature plasma incident at right angles to a threedimensional dipole, under the assumptions of a pressure balance at each point between the specularly-reflected solar wind and the magnetic field. The results of one of these studies is shown in Figure 5. The surface approximates a hemisphere on the day side, with a characteristic "dimple" at the position of the null point in the noon meridian plane. The null point is about 15-20 degrees from the pole, towards the sun. On the back side the surface becomes essentially cylindrical in shape, the diameter of the cylinder being 35-40 $R_{e}$ (assuming a subsolar boundary distance of $10 \mathrm{R}_{\mathrm{e}}$ ). Because the plasma is assumed to be at zero temperature, this theoretical magnetosphere cavity does not close off, but extends to infinity in the anti-solar direction.

Once the boundary surface has been obtained it is possible to calculate the resulting distortions in the dipole field. These distortions can be expressed best 


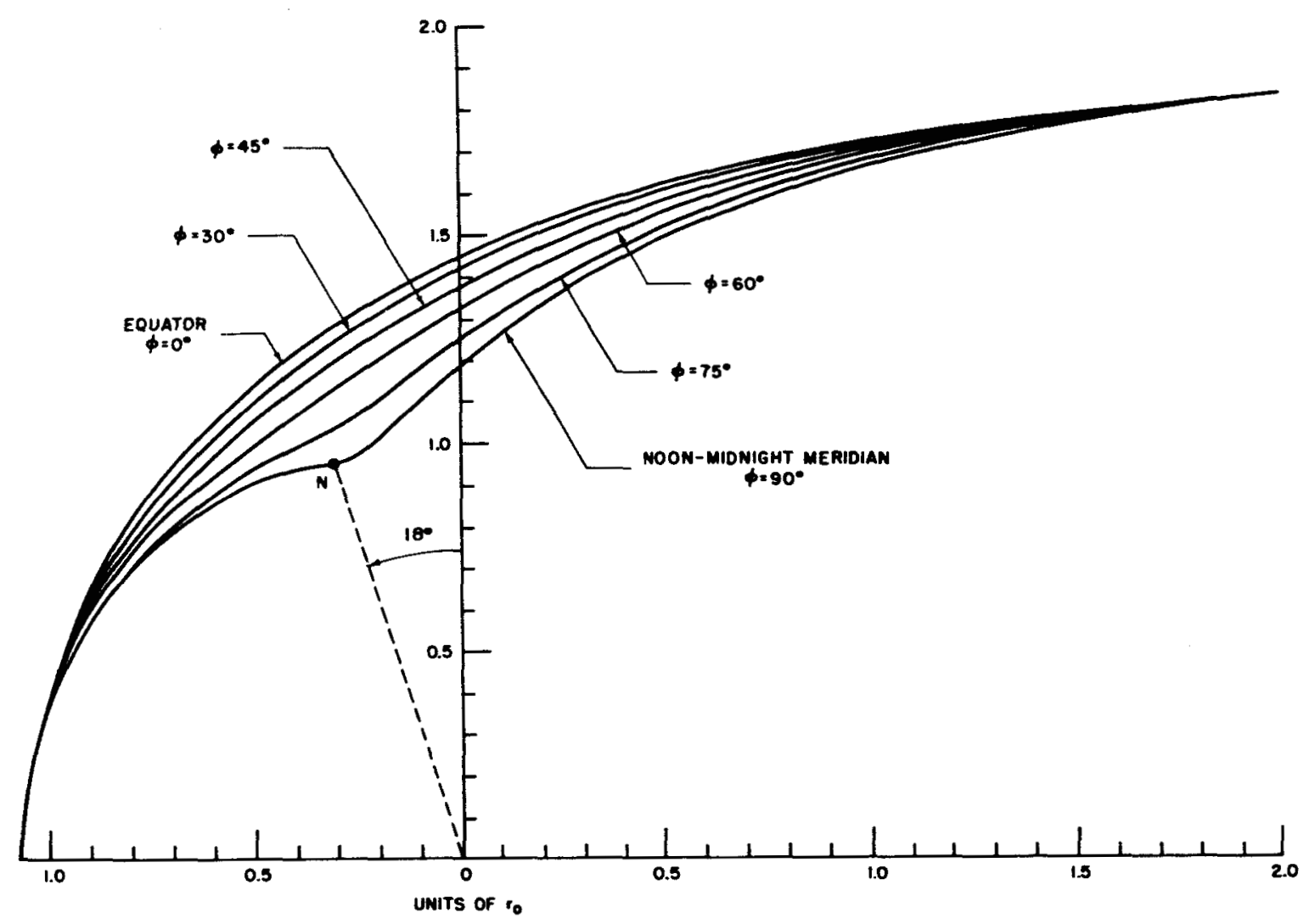

Figure 5-Theoretical shapes of the intersection of the magnetopause with planes parallel to the earth-sun line.

as a spherical harmonic expansion, the coefficients determined by analysis of the boundary surface currents. Using this expansion, the topology of the field within the cavity can be calculated everywhere. The results obtained by Mead for the shape of the field lines in the noon-midnight meridian are shown in Figure 6. ${ }^{(23)}$

These calculations are based on assumptions which are not entirely met. First of all, the solar wind is not field-free, but contains an imbedded field averaging about $5 \%$. Since the solar wind is therefore supersonic in the magnetohydrodynamic sense, a shock wave is formed ahead of the boundary. In the transition region, the solar wind flow is no longer directional, but becomes disordered and randomized.

In addition, Dungey has suggested that if the interplanetary field has a southward component, some of the earth's field lines would interconnect with the interplanetary field, thus modifying the field topology. (2) Axford and Petschek have suggested that dissipative forces near the boundary would cause the polar 


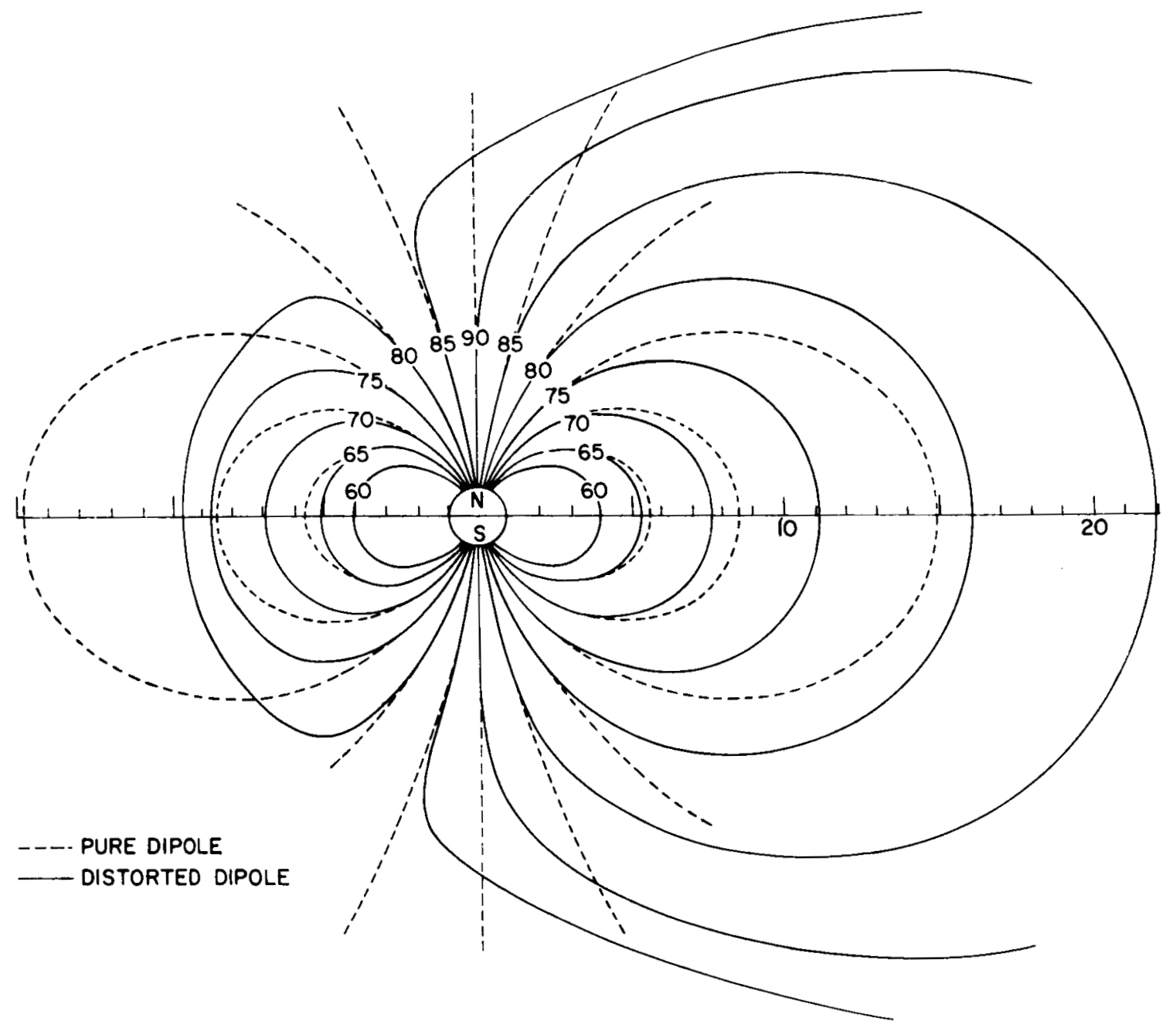

Figure 6-Theoretical shapes of distorted geomagnetic field lines in the magnetosphere noon-midnight meridian plane. The dotted lines are dipole field lines shown for comparison.

field lines to be drawn back into a very long magnetosphere tail, with the outwarddirected field lines being separated from the inward-directed ones by a neutral sheet ${ }^{(25)}$ (Figure 7). Ness has found evidence from IMP-1 data for the existence of such a sheet. (26) Dessler, ${ }^{(27,28)}$ Beard, (29) Axford and Hines, ${ }^{(30)}$ Spreiter and Jones, ${ }^{(31)}$ and others have discussed various modifications to the simple ChapmanFerraro model. Most of the discussion. however. has been qualitative, rather than quantitative in nature, because of the great difficulty in incorporating the newer ideas into a complete mathematical magnetosphere theory.

The first definite observation of the magnetospheric boundary was made with Explorer 10, launched on March 25, 1961, into a highly elliptical orbit with an apogee of 47 earth radii, approximately in the anti-solar direction (Figure 1). 


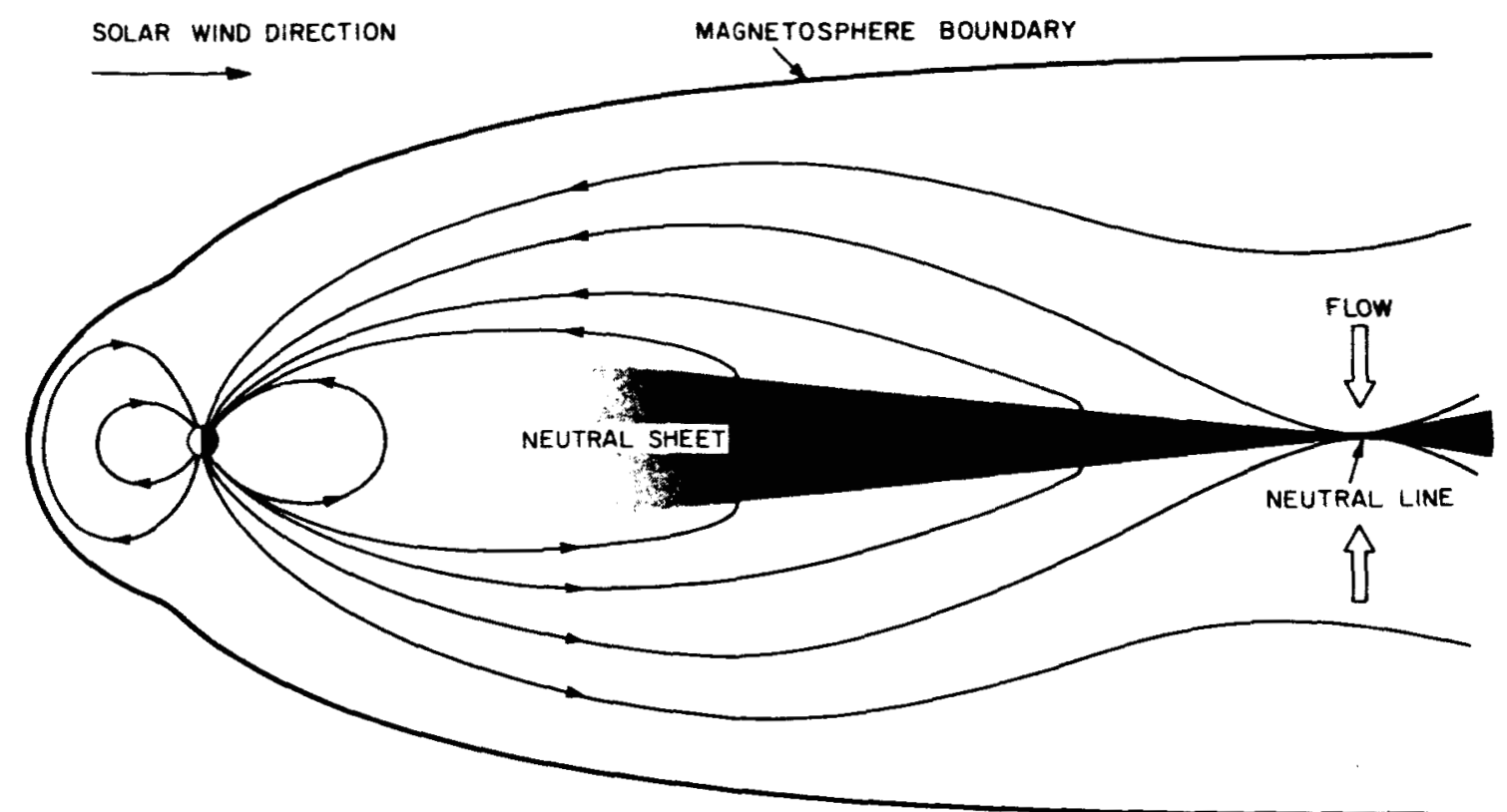

Figure 7-A theoretical model of the tail of the magnetosphere. Friction at the magnetopause pulls the field lines back and produces a neutral sheet.

Between distances of $22 \mathrm{R}_{\mathrm{e}}$ and apogee, the satellite apparently crossed the boundary (or vice versa) on six principal occasions. This conclusion was reached after comparing the results of the rubidium vapor magnetometer experiment of Heppner et al. at Goddard ${ }^{(32)}$ with the plasma probe experiment of Bridge et al.5) at MIT. While inside the magnetosphere, the magnitude of the field was comparatively strong (20-30 gammas), and there was usually no detectable plasma. Outside the boundary the field changed directions and became weaker (10-15y), and plasma was always observed. The position of the satellite at the times during which the boundary crossings were observed indicated that if the magnetosphere tail was symmetric about the sun-earth line, the dimensions of the cavity would be somewhat broader than the current theories had indicated; i.e., about $50 \mathrm{R}_{\mathrm{e}}$ in diameter, as opposed to the predicted $35-40 \mathrm{R}_{\mathrm{e}}$.

Since Explorer 10 was battery-operated, it only transmitted during its first outbound pass, and no further data was received. Explorer 12 was launched August 16,1961 , in a generally solar direction with an apogee of $13.1 \mathrm{R}_{\mathrm{e}}$ (Figure 1). A three-element flux gate magnetometer provided by Cahill at the University of New Hampshire ${ }^{(18)}$ was one of the various experiments on board. This instrument was capable of detecting the magnitude and direction of fields between 10 and 1,000 gammas. The satellite had a period of 26-1/2 hours, and, while apogee remained within about $60^{\circ}$ of the solar direction, crossings of the magnetosphere boundary was observed twice during each orbit, once on the outbound 
pass and once on the inbound pass. The most obvious characteristic of the boundary was a sudden change in direction of the magnetic field, with the direction and magnitude of the field much more variable outside the boundary. Usually, but not always, this was accompanied by a decrease in the magnitude of the field outside the boundary. An example of a typical pass is shown in Figure 4. The outer shock wave boundary was not observed, since it was usually beyond apogee (see Figure 1).

\section{The Transition Zone and Shock Wave}

The IMP-1 (Exp. 18) satellite was launched in November 1963 into a highly eccentric orbit going out to $30 \mathrm{R}_{\mathrm{e}}$ (Figure 1) with instruments on board designed to explore the outer magnetosphere and interplanetary region. This satellite discovered a new and interesting feature of the terrestrial environment. Two instruments on IMP showed the existence of a detached bow shock wave towards the sun from the magnetopause. The magnetometer flown by Ness ${ }^{(13)}$ (capable of measuring fields with a sensitivity of $1 / 4 y$ ) showed two transitions as it moved radially away from the earth. As an example on orbit 11 (see Figure 8 ) at about $13.6 \mathrm{R}_{\mathrm{e}}$ the satellite passed out through the magnetopause into a region of disordered field of from $0-15 \gamma$, with variable direction. Then at $20 \mathrm{R}_{\mathrm{e}}$ a second transition occurred, and outside this the field became quite steady at about $4 y$.

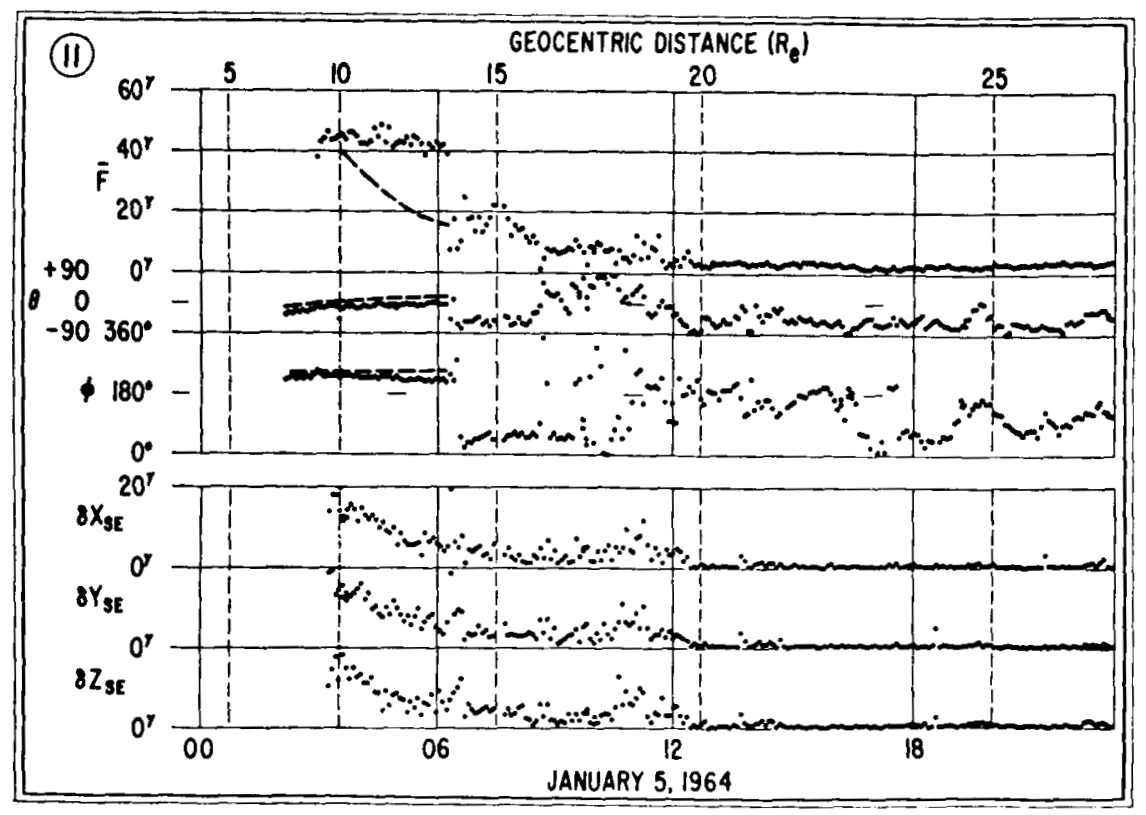

Figure 8-Magnetic field data from orbit 11 of Explorer XVIII (IMP:-1). The magnetopouse is at $13.6 \mathrm{R}_{e}$. The second transition of $20 \mathrm{R}_{\mathrm{e}}$ to an ordered field outside is the location of the bow shock wave. 
This second transition indicates a shock wave. The magnetic field in the solar wind outside the shock is rather steady and then suddenly, in a few thousand kilometers, the field changes character significantly and becomes turbulent and disordered. The variance of the field (the RMS deviation of a 5 minute set of data) is very small outside the transition and is relatively large inside the shock.

Even before the IMP-1 results were obtained, the suggestion had been made in analogy to supersonic aerodynamics, that there might be such a detached shock wave upstream of the earth. Figure 9 shows the detached shock wave ahead of a sphere immersed in a supersonic flow of gas. The analogy with the magnetosphere however is quite imperfect. In aerodynamics the shock wave results from collisions of particles and is about one mean free path thick. In the solar wind a coulomb collision mean free path $\left(=1 / \mathrm{n}=10^{+14} \mathrm{~cm}\right)$ is so large that collisions play no part in the observed shock wave. This collisionless shock wave is produced by the action of the magnetic field. and the characteristic dimension is the cyclotron radius, not the mean free path. A 1 Kev proton in a field of 10 , has a cyclotron radius of $450 \mathrm{~km}$. Inside a detached supersonic aerodynamic shock, ahead of the obstacle the regime is turbulent. This appears to be the case for the magnetospheric detached collisionless shock wave also.

The MIT plasma detector on IMP-1 also observed the shock wave. ${ }^{(9)}$ Outside the shock near apogee the detector, a multi-grid faraday cup, showed a narrow well-collimated beam of solar wind moving radially away from the sun. A typical measured proton flux was $10^{8} \mathrm{p} / \mathrm{cm}^{2}-\mathrm{sec}$. The wind usually appeared all in one energy window, e.g., from 220 to $640 \mathrm{ev}$. At the same place that the magnetometer showed the change in character of the magnetic field the solar wind also changed. Outside the shock the plasma is unidirectional flowing from the sun (see Figure 10). Inside the shock near the subsolar point the plasma is more nearly isotropic. On the sides of the magnetosphere the flow becomes more directed, flowing backwards along the sides. Besides this change in directionality the protons change in energy too. In the transition zone between the shock and the magnetopause are protons of both considerably higher and lower energy and also of lower energy than in the solar wind. All channels of the MIT detector show significant proton

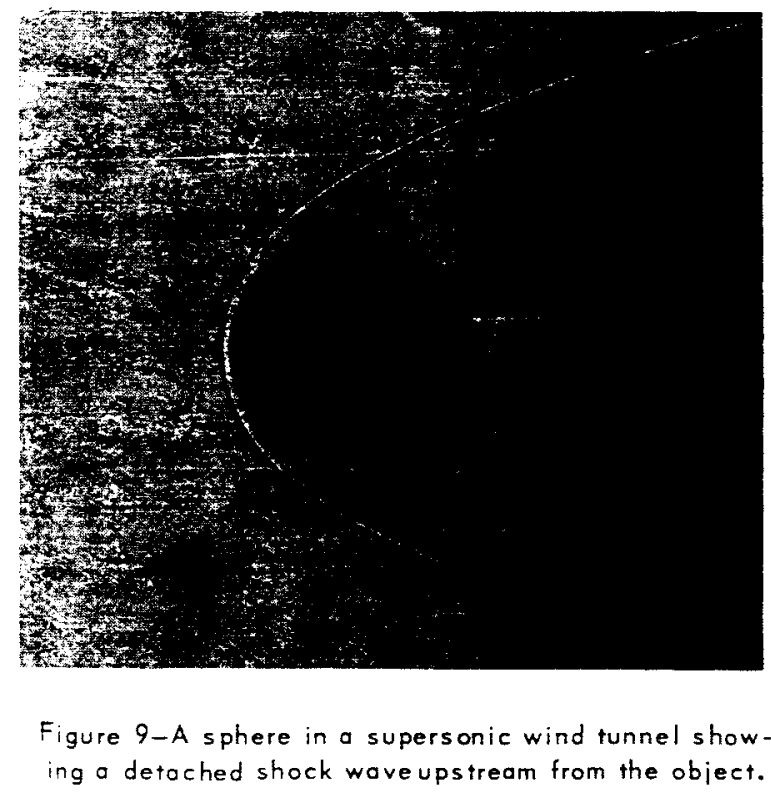


INTERPLANETARY MEDIUM

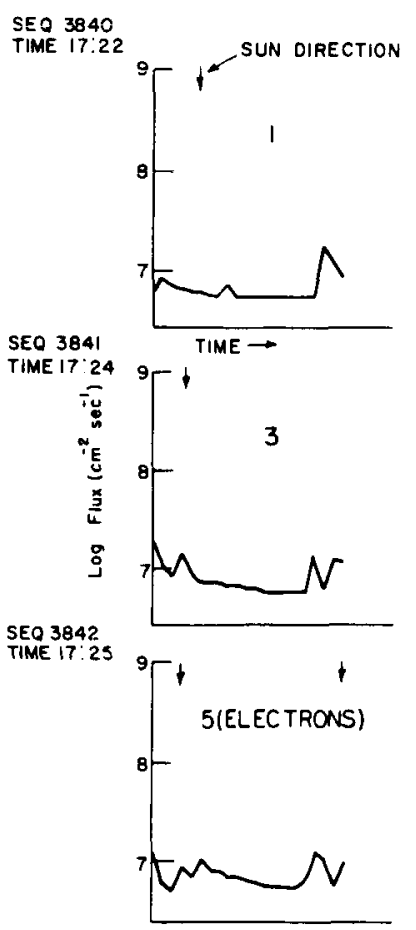

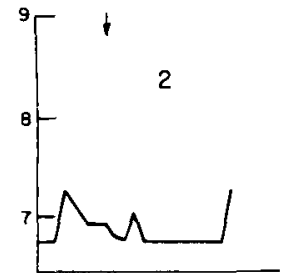
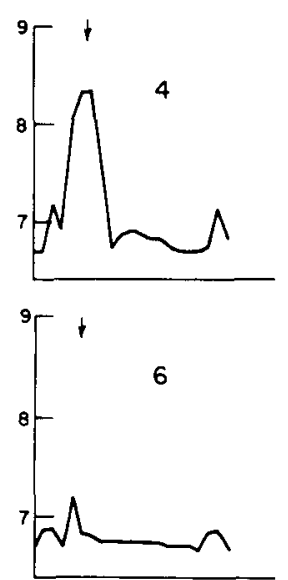

TRANSITION ZONE

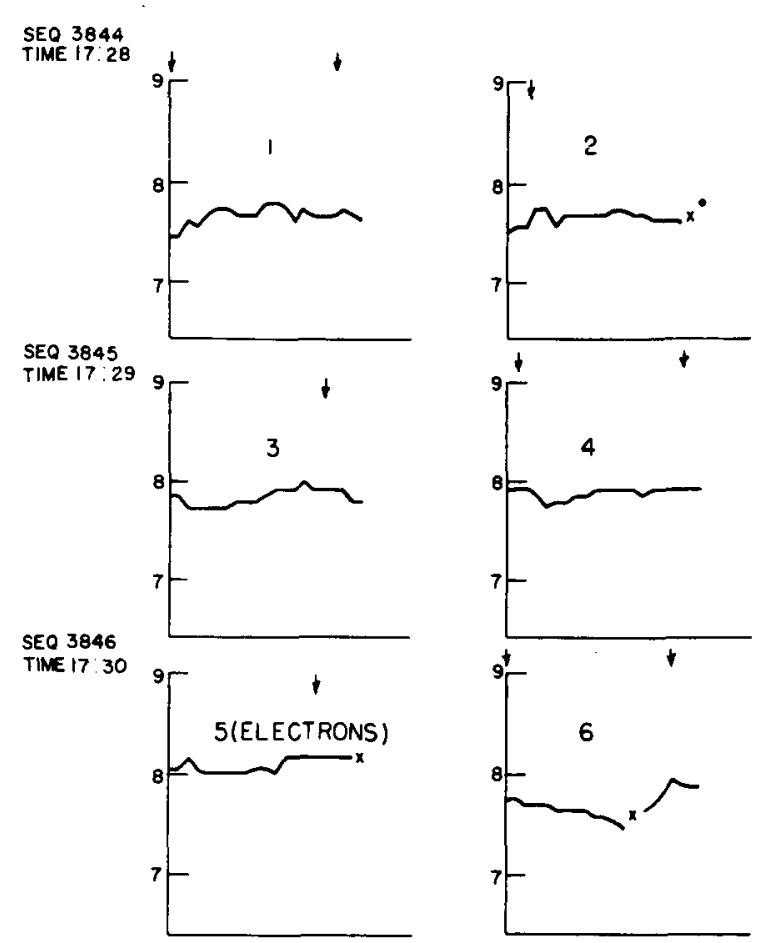

Figure 10-Solar wind data from the MIT plasma probe on IMP-I. In the interplanetary medium the detector shows large fluxes in only one energy channel. In the transition zone there are significant fluxes in all channels (including the electron one).

fluxes in the transition zone. The Ames plasma detector, ${ }^{(10)}$ a multichannel electrostatic analyzer, showed the change in proton energy too (see Figure 11). This detector indicates the flow in the transition region is somewhat anisotropic even near the subsolar point. These two experiments show that in the transition zone the proton energy spectrum extends from $0.1<\mathrm{E}<5 \mathrm{Kev}$. Apparently the plasma has been thermalized in this region. It is nearly monoenergetic outside the shock and roughly maxwellian in the transition zone. Shock waves normally produce an increase in entropy. The change in both the proton energies and angular distribution indicates an increase in disorder, and therefore an increase in entropy inside the bow shock.

Electrons have not yet been observed in the solar wind, although they must be there for the plasma to be electrically neutral. If they have the same velocity as the protons in the wind they would have an energy of about $1 \mathrm{ev}$, and no instruments so far flown would have detected them. The MIT plasma probe ${ }^{(9)}$ had a channel to count electrons of $65<\mathrm{E}_{\mathrm{c}}<210 \mathrm{ev}$. It detected no electrons outside the shock, but inside the shock a flux of $\sim 10^{8}$ electrons $/ \mathrm{cm}^{2}-\mathrm{sec}$ in this energy range was usually found. These are apparently solar wind electrons accelerated 
in the transition zone. Freeman earlier had detected a flux of $\sim 10^{10}$ electrons/ $\mathrm{cm}^{2}-\mathrm{sec}$ of $200 \mathrm{ev}<\mathrm{E}_{\mathrm{e}}<500 \mathrm{kev}$ outside the magnetosphere with a CdS detector on Explorer XII. ${ }^{(33)}$ This energetic electron flux extended out about $20,000 \mathrm{~km}$ beyond the magnetopause on to just about the shock position. It seems quite apparent that these electrons are the same population observed by the MIT plasma probe on IMP. The Goddard retarding potential analyzer on IMP-1 also detected a substantial electron flux in the transition zone.(34) The flux measured by this instrument was isotropic and consisted of $10^{8}$ electrons/ $\mathrm{cm}^{2}-\mathrm{sec}$ of $\mathrm{E}>100 \mathrm{ev}$.

The magnetopause may not really exclude all the plasma striking if from outside. Measurements by Serbu on IMP show that the electron flux in the energy range $5<\mathrm{E}<100 \mathrm{ev}$ does not show a discontinuity at the magnetopause, while the solar wind proton flux of Bridge does fall off sharply inside this boundary. The boundary seems to be semi-permeable, allowing electrons to flow inwards, but not protons. This would suggest certain instabilities at the boundary which are mass-sensitive.

Higher energy electrons were observed on IMP-1. A solid state detector of Simpson ${ }^{(35)}$ and a Geiger counter of Anderson ${ }^{(36)}$ showed that fluxes of $\mathrm{E}>40 \mathrm{KeV}$ electrons were present intermittently. Anderson found peaks of $\varphi^{\sim} 10^{6}$ electrons/ $\mathrm{cm}^{2}-\mathrm{sec}$ lasting the order of minutes (see Figure 12). These usually occurred in the transition zone close to the magnetopause. None were observed near apogee on early orbits. Anderson has suggested that these particles are sloughed off from the magnetosphere and had previously been trapped particles. Simpson suggested they were at the shock location and might be locally accelerated in the shock. Jokipii and Davis ${ }^{(37)}$ have showed that it is unlikely that the particles would be observed at the shock location. Acceleration by a factor of 2 or 3 is possible at the shock, but this is clearly not enough to produce $40 \mathrm{kev}$ electrons. The particles should be carried along with the bulk velocity of the solar wind and should be observed at all places downstream of the source location, not just at the shock. Jokipii suggests the particles may be Fermi-accelerated in the 


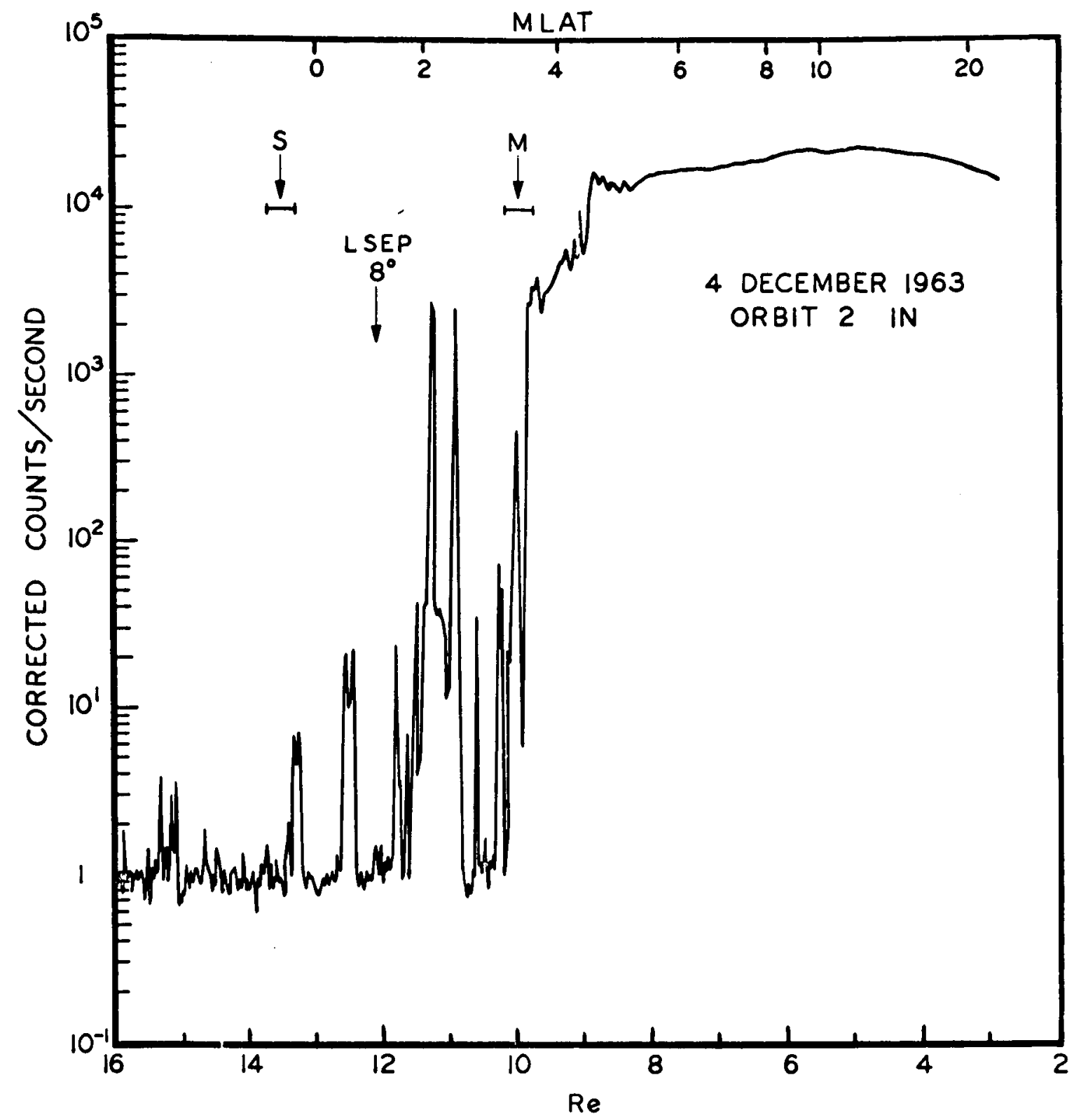

Figure 12-Flux of E $>40 \mathrm{Kev}$ electrons detected on IMP. The radiation belt is present inside the magnetopause of $10 R_{e}$, and outside this occasional "islands" of particles are observed.

transition zone, and also that the magnetic field geometry may be such that local trapping occurs to produce large local fluxes resembling the observed spikes. There is no quantitative theory of the origin of these energetic electrons.

The electron measurements described here all were made at small SEP (sun-earth-probe) angles. Data taken at larger SEP angles show other features 
of the shock and transition zone. Figure 13 shows the location of the magnetopause and shock as measured by the magnetometer for the first 48 orbits of IMP-1. The curve through the magnetopause points is the theoretical shape of the boundary as calculated by the single-particle reflection model. The agreement is quite good at least to $90^{\circ} \mathrm{SEP}$. At larger angles, the magnetopause is observed to flare out more than the single-particle theory predicts. The curve through the shock wave points is the theoretical curve for an aerodynamic shock wave of mach number 8 for a gas of $\gamma=5 / 3$. In this plasma situation the mach number is replaced by the Alfven number $=V_{w} / V_{A}$. For the interplanetary medium the Alfvén velocity is roughly $V_{A}=50 \mathrm{~km} / \mathrm{sec}$, so the Alfvén number of the solar wind is about 8.

The interplanetary field measured on IMP-1 was usually quite steady in magnitude at about $4 \%$. On orbit 5 the field was unusually disturbed, and rose

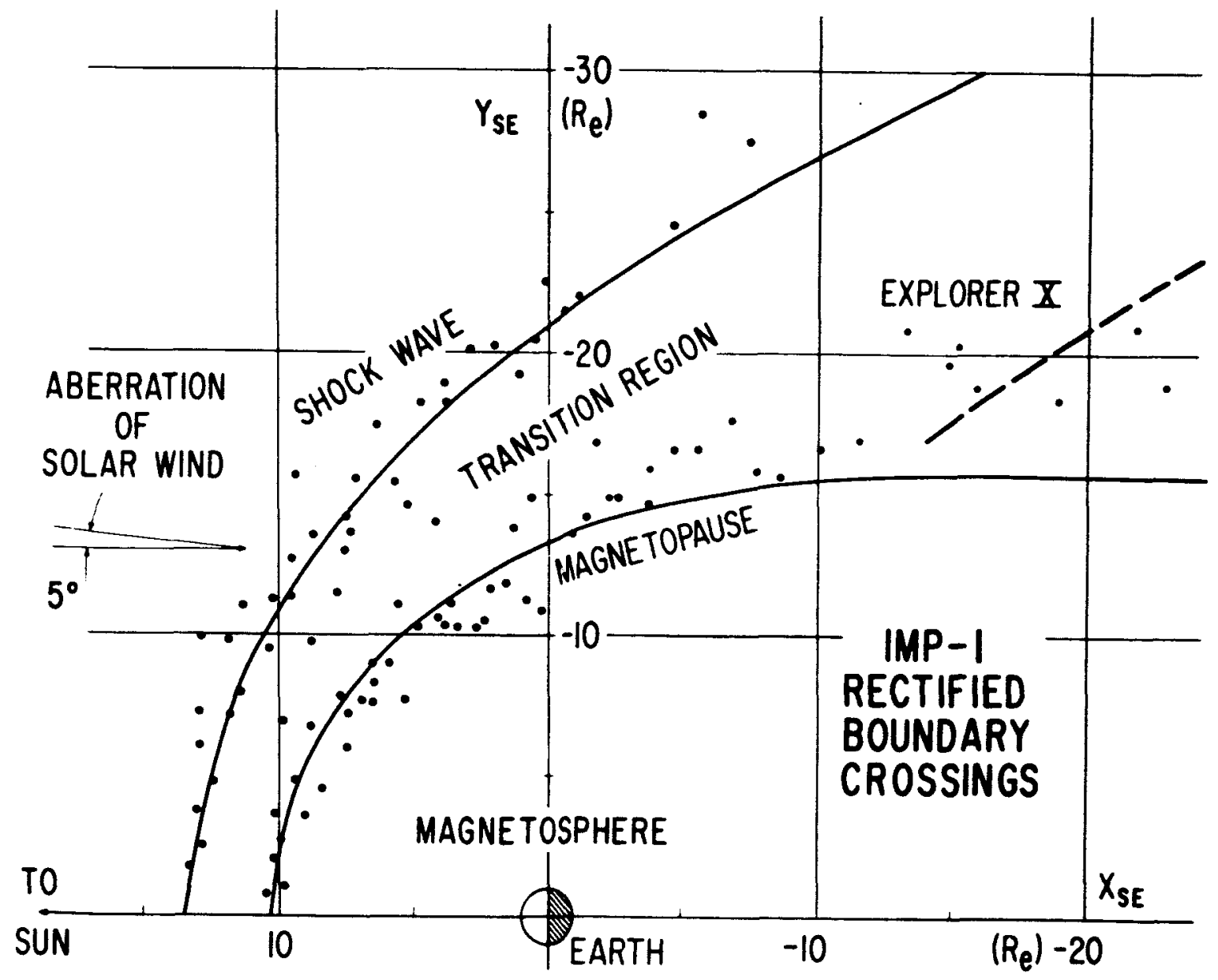

Figure 13-The location of the magnetopause and bow shock as determined by the magnetometer on IMP-1. Shown for comparison are the theoretically expected locations. 
to above $10 \gamma$ for a day or more. On the day this happened the moon nearly eclipsed the satellite, as shown in Figure 14. Ness has attributed this disturbed field to a wake of the moon..$^{(37 a)}$

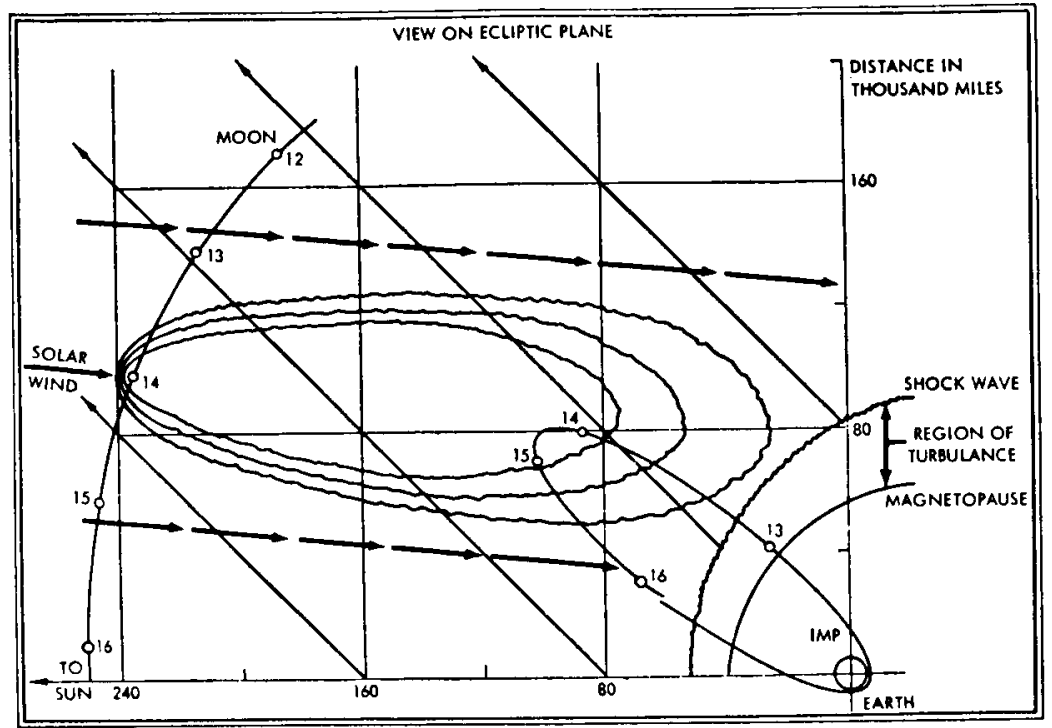

Figure 14-On December 14 and 15 the interplanetary magnetic field measured by IMP.1 was unusually disturbed. This was the period of closesteclipse by the moon and may indicate a magnetic tail behind the moon as shown here. 
III.

\section{RADIATION BELTS}

\section{A. Discovery of the Belts}

In 1958, when Explorer I was launched with a geiger counter on board, it discovered a region of high count rate starting at about $1000 \mathrm{~km}$ altitude. This was unexpected. In fact, it was suggested that the counter might have malfunctioned. But when Explorer III showed the same results a little later, it was demonstrated that the effect was real. Van Allen, who had conducted the experiments on Explorer I and Explorer III, realized very soon that the measured high count rates were due to charged particles trapped in the earth's magnetic field. ${ }^{(38)}$ Störmer had worked extensively on this general subject ${ }^{(39)}$ and even calculated orbits of trapped particles years earlier, but the actual existence of trapped particles had not been suggested in this work. Singer's ideas about the existence of a terrestrial ring current had also essentially included the idea of trapped particles. ${ }^{(40)}$

At the same time that these experiments in space were going on, experiments with trapped particles were being conducted in various laboratories. Project Sherwood is an attempt by the AEC to make a controlled thermonuclear reaction on a small scale by confining charged particles in a magnetic field. Christofilos, who was working on Sherwood, extrapolated the laboratory idea to earth scale and suggested the possibility of trapping a large number of charged particles in the magnetic field of the earth by using a nuclear explosion to inject the particles. (41) This idea was carried out in the Argus experiment and demonstrated experimentelly that charged particles could really be trapped in the earth's field.

From a study of the Explorer I data, Van Allen showed that the particles counted were geomagnetically trapped. Data taken at different longitudes looked quite different when plotted in terms of geographic coordinates, but when replotted in terms of geomagnetic coordinates the different sets of data agreed. Later McIlwain developed an especially useful set of magnetic coordinates, the B-L system, which is now normally used in plotting radiation belt data. ${ }^{(42)}$ This system takes data collected in geographic coordinates and combined data at different longitudes to make a two dimensional presentation of the data. In a dipole field $L$ is the geocentric distance to the equatorial crossing of a field line in units of earth radii and $B$ is the value of the magnetic field strength. For the earth's field the definition of $\mathrm{L}$ is more complicated but fundamentally similar. 
Sputnik III in May 1958 confirmed the existence of the trapped radiation with measurements up to $1800 \mathrm{~km} .^{(13)}$

Explorer IV was launched in July 1958 with instrumentation to study the natural radiation belt and also to study the artificial belt produced by the Argus nuclear tests. A map of the intensities of the radiation belt up to an altitude of $2200 \mathrm{~km}$ was obtained by Explorer IV as well as a range spectrum of the charged particles. ${ }^{(4)}$

Pioneer III in December $1958^{(45)}$ and Pioneer IV in March $1959^{(46)}$, and also the Soviet Space Rocket I in January 1959 and Space Rocket II in September $1959^{(43)}$, gave isolated passes more or less radial outwards through the radiation belt.

The data from Explorer IV and Pioneer III allowed Van Allen ${ }^{(45)}$ to show the existence of two radiation belts (see Figure 15). This is really only the case for particles thatcan penetrate $1 \mathrm{gm} / \mathrm{cm}^{2}$, and we know now that the two zones

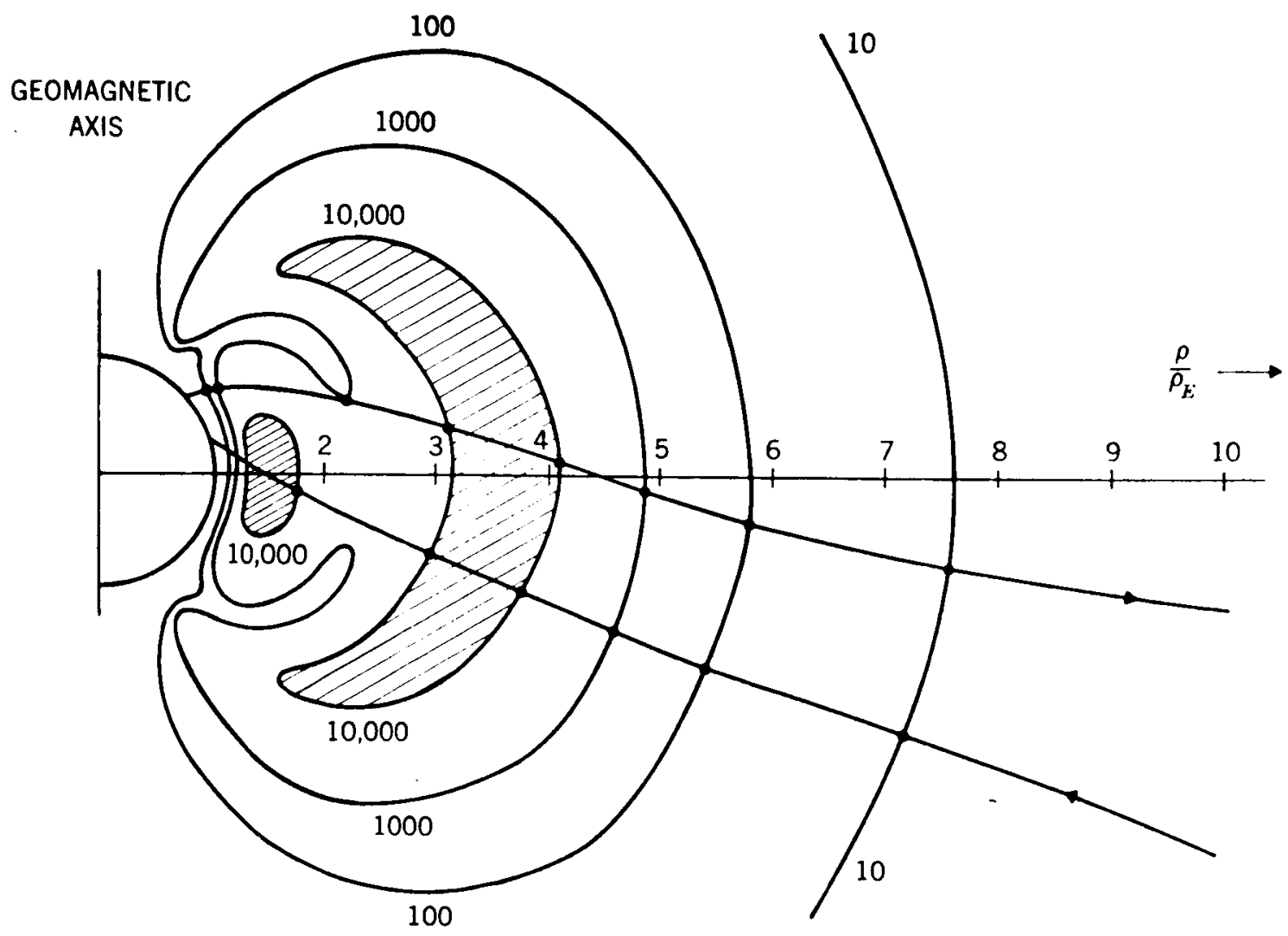

Figure 15-Van Allen's picture of the inner and outer zone of the radiation belt mode ofter Pioneer 11 l. 
are made up of different kinds of particles-protons in the inner zone and electrons in the outer zone. A comparison of Pioneer III and IV data showed the time variability of the outer zone.

The data obtained by these various satellites before 1960 enabled one to give general spatial limitations and time variability of the radiation belt and say something about the penetrability of the radiation, but one did not know what kind of particles were being counted.

The general problem of charged particle motion in a dipole field is complicated. Fortunately, for radiation belt particles, an approximation can be used which simplifies the situation considerably. Alfvén introduced the idea of the guiding center of a particle. The particle motion here is described in terms of (1) a rapid gyration about a field line with a cyclotron period $T_{\text {c and adius of }}$ gyration $R_{c}$ and (2) motion of the guiding center of the particle along the line of force. The motion along the line is periodic too. The particle is reflected by the converging magnetic field near the earth and bounces back and forth in the exosphere with a bounce period $T_{B}$. There is another motion, a slow drift in longitude around the earth with a period of revolution of $T_{R}$. Particles at $2000 \mathrm{~km}$ altitude near the equator will have the following characteristics:

\begin{tabular}{rrrrrrr}
\hline & \multicolumn{1}{c}{$\mathrm{R}_{\mathrm{c}}$} & \multicolumn{1}{c}{$\mathrm{T}_{\mathrm{c}}$} & \multicolumn{1}{c}{$\mathrm{T}_{\mathrm{B}}$} & \multicolumn{1}{c}{$\mathrm{T}_{\mathrm{R}}$} \\
\hline 50 kev Electron & $5 \times 10^{3} \mathrm{~cm}$ & $2.5 \times 10^{-6} \mathrm{sec}$ & $0.25 \mathrm{sec}$ & 690 & min \\
1 Mev Electron & $3.2 \times 10^{4} \mathrm{~cm}$ & $7 \times 10^{-6} \mathrm{sec}$ & $0.10 \mathrm{sec}$ & 53 & min \\
1 Mev Proton & $1 \times 10^{6} \mathrm{~cm}$ & $4 \times 10^{-3} \mathrm{sec}$ & $2.2 \mathrm{sec}$ & 32 & min \\
10 Mev Proton & $3 \times 10^{6} \mathrm{~cm}$ & $4.2 \times 10^{-3} \mathrm{sec}$ & $0.65 \mathrm{sec}$ & 3.2 & $\mathrm{~min}$ \\
500 Mev Proton & $2.5 \times 10^{7} \mathrm{~cm}$ & $6 \times 10^{-3} \mathrm{sec}$ & $0.11 \mathrm{sec}$ & $0.084 \mathrm{~min}$ \\
\hline
\end{tabular}

Because the three periods are so different, the particle motion is separable into these three components. If the cyclotron radius of the particle $R_{c}$ gets comparable to the diameter of the earth, the motion is not separable, but even for $1 \mathrm{Bev}$ protons this condition is not attained.

\section{B. The Inner Radiation Belt}

The first experiment in the radiation belt that unambiguously identified the particles counted was conducted with a stack of nuclear emulsions on a Atlas rocket in April, 1959. The emulsion stack was recovered and developed and the nuclear tracks read. The range and ionization of the particles were measured, the particles identified, and their energies determined. 


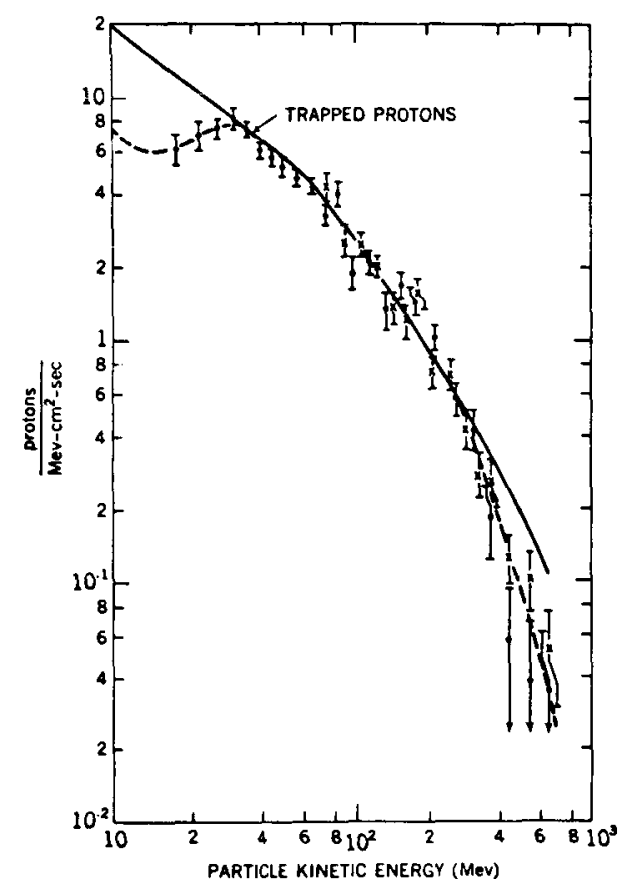

Figure 16-Energy spectrum of protons in the inner radiation zone as measured by nuclear emulsions.
Protons of $\mathrm{E}>75 \mathrm{Mev}$ and electrons of $\mathrm{E}<12 \mathrm{Mev}$ could get through the $6 \mathrm{gm} / \mathrm{cm}^{2}$ shielding into the nuclear emulsions. No electrons, but a large number of protons were found. The energy spectrum of protons measured by Freden and White ${ }^{(48)}$ on a later flight is shown in Figure 16. Other experiments have shown very similar energy spectra and intensities of protons and have extended the data down to lower energies. (49,50) Below about $40 \mathrm{Mev}$ the energy spectrum becomes quite flat and shows a slight dip at about $20 \mathrm{Mev}$. The inner belt proton flux seems rather constant in time, varying by less than a factor of 2 for several flights. This indicates that the particle lifetime is quite long.

Mcllwain used the spatial distribution of $\mathrm{E}>30 \mathrm{Mev}$ protons in the inner zone as measured on Explorer IV to develop the B-L magnetic coordinate system. Figure 17 shows the $\mathrm{B}-\mathrm{L}$ map of inner zone protons of $\mathrm{E}>30 \mathrm{Mev}$. In geographic coordinates this data is a mess.

We now have a quantitative interpretation of the high energy protons found by Freden and White. The protons are made by the decay of neutrons leaking out of the atmosphere of the earth..$^{(43,51,52)}$ The neutrons are generated in the atmosphere by cosmic ray protons colliding with $\mathrm{O}$ or $\mathrm{N}$ nuclei. When the escaping neutrons decay, they make protons, which can be trapped to form part of the radiation belt. The trapped protons are eventually lost by interacting with the thin atmosphere at high altitudes. This model, when studied quantitatively, gives good agreement with the measured proton flux and energy spectrum above $30 \mathrm{Mev} .{ }^{(53)}$ Solar cycle time changes in the inner edge of the energetic protons are expected, due to the change in atmospheric density with solar cycle and the resulting change in the loss rate of the protons. ${ }^{(54)}$ Changes observed on Explorer VII for $\mathrm{L}<1.7$ seem to agree with this idea. ${ }^{(55)}$

Naugle and Kniffen flew emulsions on the NERV vehicle to study the energetic inner zone protons. ${ }^{(56)}$ They found, besides the Freden and White type protons a lower energy group ( $\mathrm{E}<50 \mathrm{Mev}$ ) near the outer edge of the radiation belt for $\mathrm{L}>1.7$ (see Figure 18). These protons may very well come from decay of neutrons produced in the polar atmosphere by solar protons.(4)) They would have lower energy and be limited to high latitudes as observed. A study by Lenchek ${ }^{(57)}$ gives reasonable agreement with the data. 


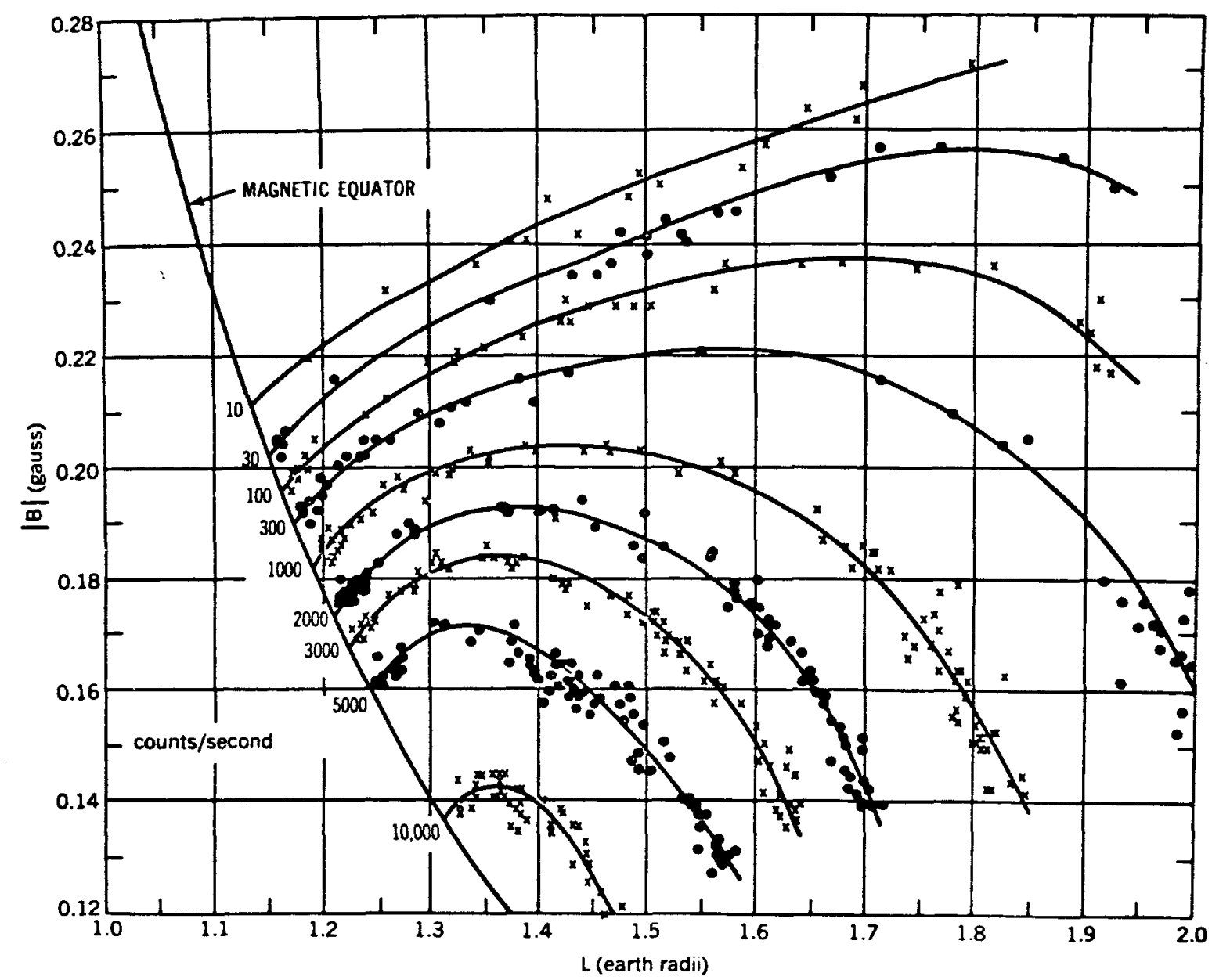

Figure 17-A map showing the spatial distribution of energetic protons ( $>30 \mathrm{Mev}$ ) in the inner zone of the radiation belt. This map uses Mcllwain's $B-L$ coordinate system.

Recently McIlwain on Explorer XV has found a second maximum at $\mathrm{L}=2.2$ in the radial distribution of energetic protons ${ }^{(58)}$ of $E_{p}>40 \mathrm{Mev}$ (see Figure 19). This outer particle group was stable for some months and then was changed suddenly in a substantial way at the time of a large magnetic storm. At the outer edge of the distribution $\mathrm{L}>2.3$ the flux decreased considerably. ${ }^{(59)}$ The source of this second group of protons is uncertain. It might be neutron decay injection modified by magnetic disturbances. It might also be the result of $L$ drift from the magnetopause inward-the same process that has been invoked to explain the low energy outer zone protons observed by Davis. It is clear that some form of magnetie disturbances are important in controlling the outer edge of the energetic proton distribution.

It would seem that we need at least three sources to explain the spatial distribution and energy spectrum of $\mathrm{E}>10 \mathrm{Mev}$ protons. 


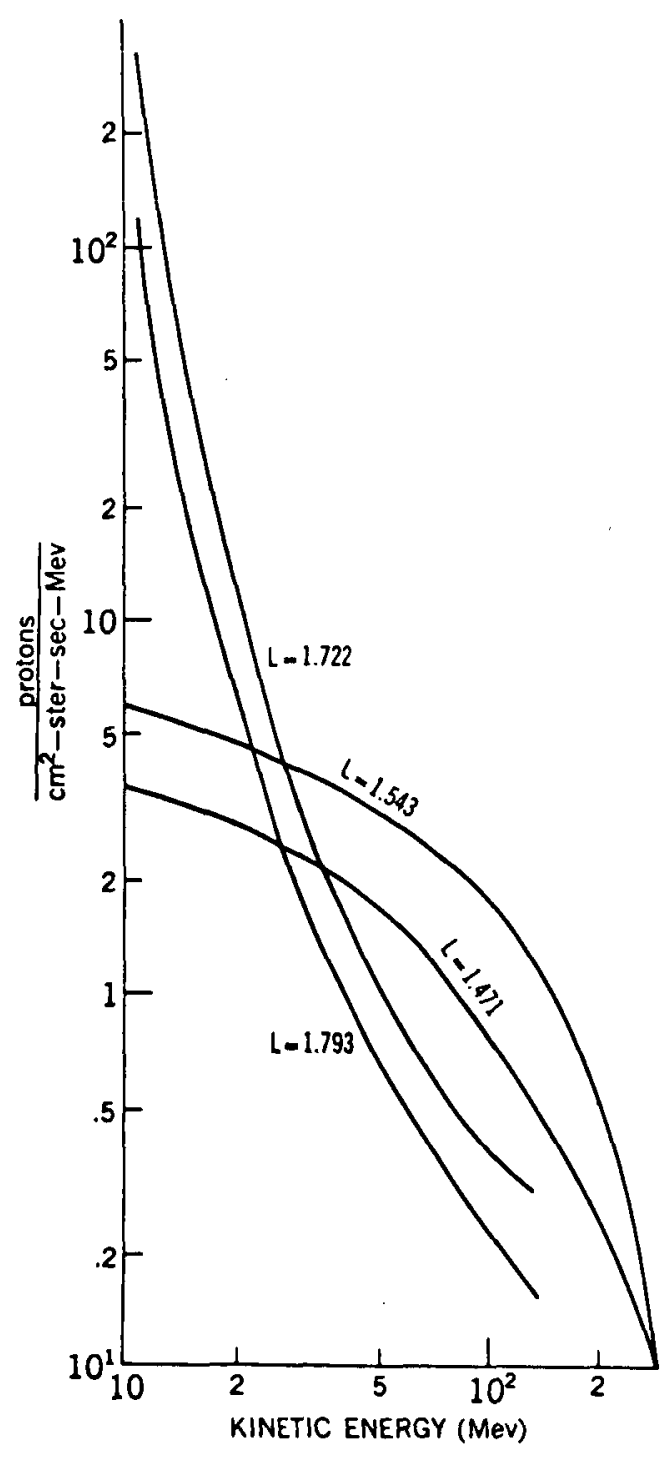

Figure 18-The proton energy spectrum of different locations in the inner zone showing the lower energy protons present in the outer parts of the inner zone.
We know rather little about the natural electrons in the inner radiation zone. It appears that there are few if any electrons of $\mathrm{E}>1 \mathrm{Mev} .^{(60)}$ From studying artificial radiation belts we know the electron lifetimes are as long as years at the center of the inner zone. These two facts support the thesis that neutron decay is an important source for electrons in the inner belt as well as protons. But there are large numbers of $\mathrm{E}<100 \mathrm{Kev}$ electrons in the inner belt. These cannot be reasonably attributed to neutron decay and imply that some other source is important too.

Freeman on Injun I found a large flux of low energy protons in the inner belt. ${ }^{(61)}$ Energy fluxes as large as $100 \mathrm{ergs} / \mathrm{cm}^{2}-\mathrm{sec}$ were observed. These particles may be the seat of the ring current (see discussion in Section IV).

\section{Artificial Radiation Belts}

Seven artificial radiation belts have been made by the explosion of high altitude nuclear bombs since 1958. These artificial belts result from the release of energetic charged particles, mostly electrons, from the nuclear explosions..$^{(62)}$ These seven explosions are found in the table on the following page. The Argus explosions were carried out to study the trapping of particles in the earth's magnetic field. Christofilos had suggested that electrons from, ${ }^{2}$ decay of fission fragments from a nuclear explosion should be able to make an artificial

radiation belt.(41) All seven artificial belts to date have this as the source. The Argus belts, studied by Van Allen on Explorer IV ${ }^{(6)}$ decayed in a week or so. During this time they did not move radially a measureable amount.

The Starfish explosion made a much more extensive belt than Argus. Electron fluxes of $10 \% / \mathrm{cm}^{2}-\mathrm{sec}$ were produced. The belt was studied at early times 


\begin{tabular}{|l|l|c|c|c|}
\hline Explosion & \multicolumn{1}{|c|}{ Locale } & Time & Yield & Altitude \\
\hline Argus I & South Atlantic & 1958 & $1 \mathrm{kt}$ & $300 \mathrm{miles}$ \\
Argus II & South Atlantic & 1958 & $1 \mathrm{kt}$ & $300 \mathrm{miles}$ \\
Argus III & South Atlantic & 1958 & $1 \mathrm{kt}$ & $300 \mathrm{miles}$ \\
Starfish & $\begin{array}{l}\text { Johnson Island, } \\
\text { Pacific Ocean }\end{array}$ & July 9, 1962 & $1.4 \mathrm{Mt}$ & $400 \mathrm{~km}$ \\
USSR & Siberia & Oct. 22, 1962 & Several hundred kt & $?$ \\
USSR & Siberia & Oct. 28, 1962 & $?$ & $?$ \\
USSR & Siberia & Nov. 1, 1962 & $?$ \\
\hline
\end{tabular}

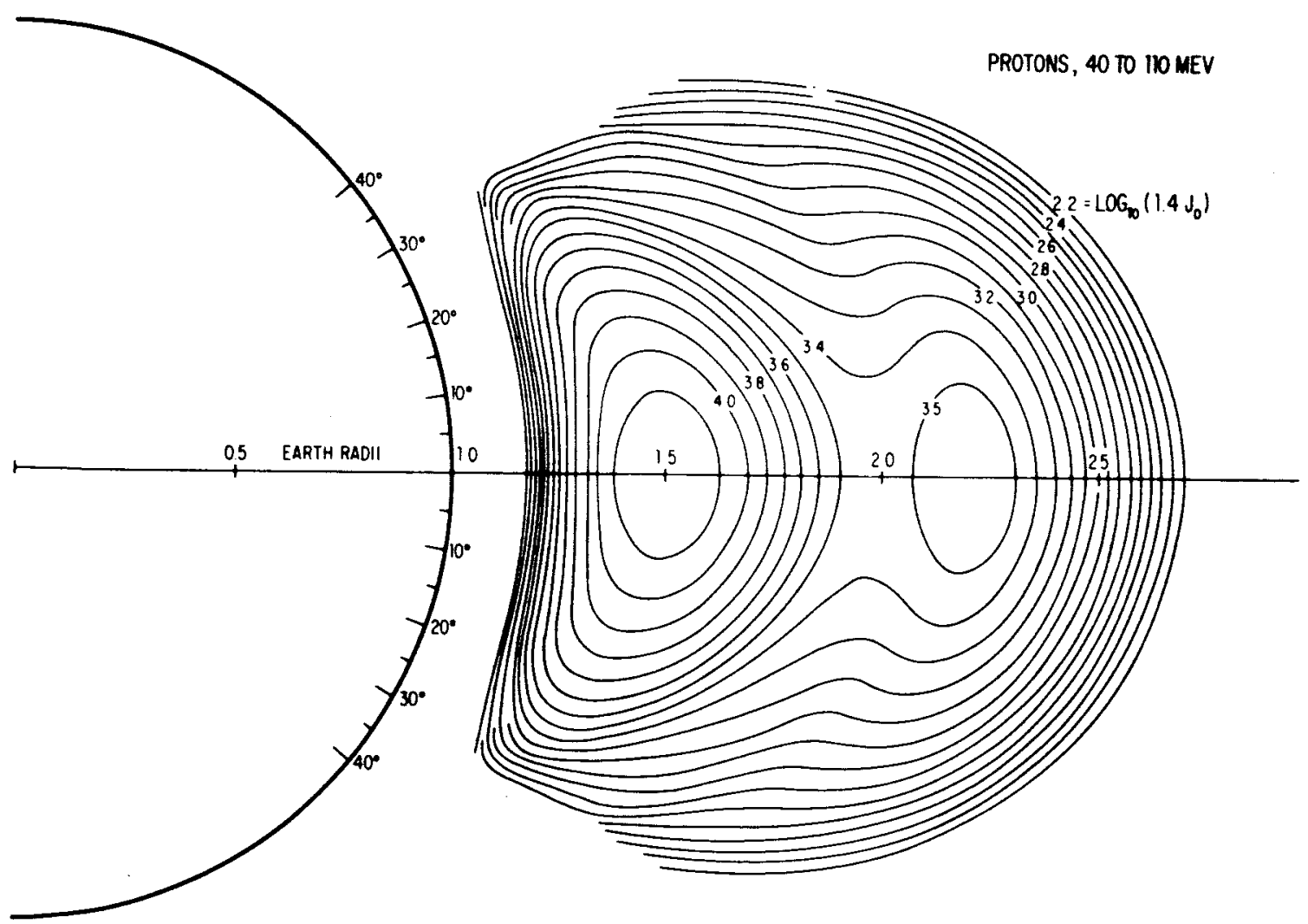

Figure 19-Spotial distribution of high-energy protons measured by Mcllwain on Explorer XV, showing the double-peaked distribution. (This is an R-Amagnetic coordinate map os obtained from transformation of $B . L$ coordinotes, and is not the same as a geographic map.) 


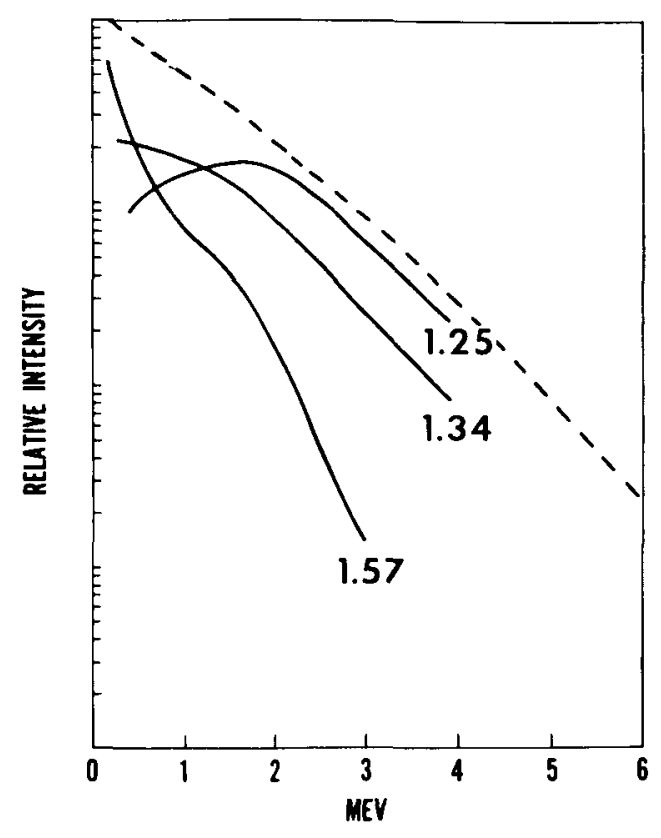

Figure 20-Energy spectra of electrons in the Starfish artificial radiation belt as measured on sotellite $1962 \mathrm{Bk}$. by detectors on Injun, (64) TRAAC ${ }^{(65)}$ Telstar, ${ }^{(66)}$ Ariel ${ }^{(67)}$ and Cosmos 5. ${ }^{(68)}$ Early flux maps produced by Injun ${ }^{(64)}$ and Telstar ${ }^{(66)}$ seemed to be in disagreement as to the radial extent of the belt, but now that we understand the electron energy spectra at different locations in the belt the differences are understood. The spectra were measured by West ${ }^{(69)}$ using an electron spectrometer on the Starad satellite (see Figure 20). He found the electrons at $\mathrm{L}=1.25$ had roughly a fission energy distribution, but that at $\mathrm{L}=1.5$ the electrons were of appreciably lower energy. These low energy electrons would not be measured efficiently by the Injun detector. We do not understand why the Starfish electrons extended as far away from the explosion site as they did, and we have only untested ideas as to why the electron energies were different in different sections of the artificial belt.

Brown measured the decay of the Starfish electrons with Telstar ${ }^{(66)}$ and found unusually rapid decay above $\mathrm{L}=1.7$. For $\mathrm{L}<1.5$ the decay is quite slow and obviously controlled by the atmosphere. Injun ${ }^{(69)}$ (see Figure 21) and Alouette ${ }^{(70)}$ measurements of the decay give lifetimes up to several years for low altitudes, in agreement with calculations of Welch et al. ${ }^{(71)}$ and Walt ${ }^{(72)}$ based

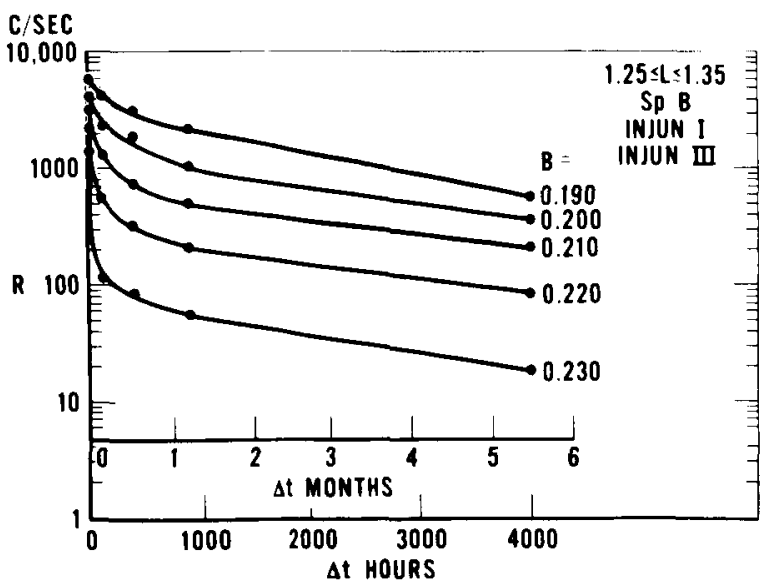

Figure 21-Decay of the Starfish ortificial radiation belt electrons at low altitudes as measured on Injun. on atmospheric scattering. Brown measured the lifetime of less than a week at $\mathrm{L}=2.5$ (see Figure 22). The satellite Explorer XV was launched to study artificial belts by following the decay of the Starfish belt. In the fall of 1962, three Soviet artificial belts were made in the region $1.7<$ $\mathrm{L}<3.0$. Explorer $\mathrm{XV}$ observed these belts and determined their time histories. All of these belts decayed rapidly, as did the Starfish belt for $\mathrm{L}>1.7$. The short lifetime at high altitude is very probably due to some type of electromagnetic waves interacting with the electrons. Dungey ${ }^{(7.3)}$ 


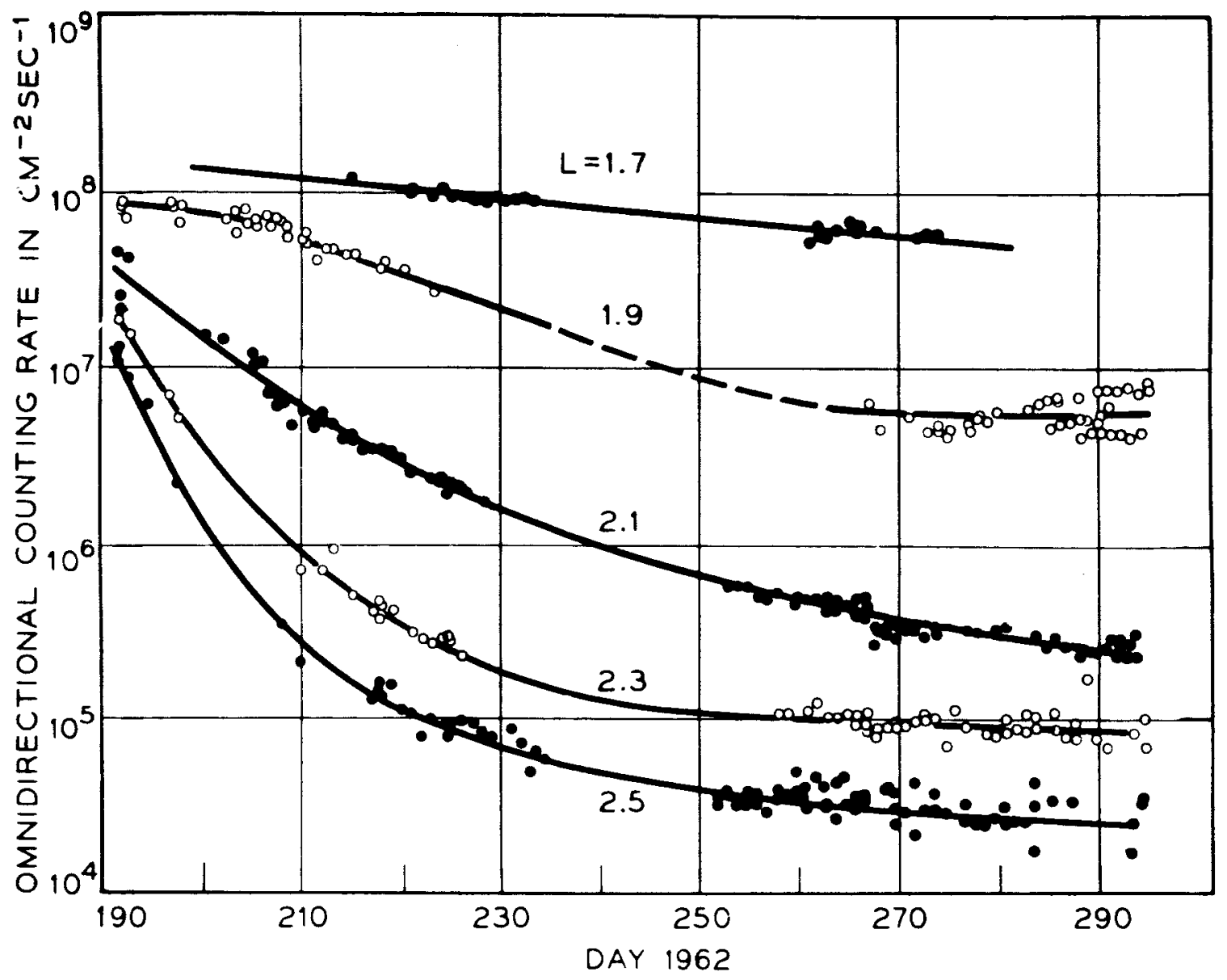

Figure 22-Rapid decay of the Starfish electrons at high altitudes as measured on Telstar.

has suggested that whistlers, circularly-polarized waves that travel along field lines, can interact resonantly with the electrons. As a result the electrons would be scattered and lost more rapidly. This seems reasonable, but cannot be considered proven. Electron lifetimes determined from watching the decay of artificial belts are the only reliable lifetimes we have. Using these values we can understand some of the characteristics of the natural trapped electrons.

Yagoda, ${ }^{(74)}$ studying protons by emulsions on low altitude polar satellites, found that the $\mathrm{E}>55 \mathrm{Mev}$ protons in the Van Allen belt were disturbed by the Starfish test. Increased proton fluxes were found at several hundred kilometers after the explosion. These fluxes decayed back to normal in a matter of months.

D. The Outer Radiation Belt

Although there is no clear demarcation between an inner and outer radiation belt for electrons, the separation is very sharp for protons and occurs at about 
$\mathrm{L}=2$. In the inner zone, there are protons up to hundreds of Mev. In the outer zone, proton energies are much lower, fluxes are much higher, and albedo neutrons are inadequate by many orders of magnitude for a satisfactory accounting of the observed fluxes.

These outer zone protons were discovered in 1962 by Davis and Williamson ${ }^{(75)}$ with equipment on Explorer 12. Observations of these protons have also been made on Explorers 14 and 15. A rather surprising result of measurements over a few years has been the stability of a major fraction of these protons, although fluxes are large and proton lifetimes relatively short.

Because of this stability, it has been possible to obtain relatively detailed information on the energy spectra and directional fluxes. The detectors measured protons with energies between $100 \mathrm{Kev}$ and $5 \mathrm{Mev}$. Most of the protons were near the lower energy limit. The data was adequately ordered through the use of $\mathrm{L}$ and equatorial pitch angle (EPA), as calculated with the Jenson and Cain earths magnetic field, ${ }^{(76)}$ for $\mathrm{L}<5$. For EPA $=90^{\circ}$, a peak intensity of $3.7 \times 10^{7}$ protons $/ \mathrm{cm}^{2}$-sec-ster has been found, with a gradual falloff in intensity at both larger and smaller $\mathrm{L}$. At any $\mathrm{L}$, the peak intensity was found for $\mathrm{EPA}=90^{\circ}$, with a smooth fall off to zero at small EPA.

The energy spectra were found to have large but smooth variations with both L and EPA (see Figure 23). More energetic protons were found near the earth and at large EPA. The spectral data was well represented by $e^{-E / E_{0}}$ where $\mathrm{E}_{0}$ varied as $\mathrm{L}^{-3}$ for $\mathrm{EPA}=90^{\circ}$ and varied less rapidly with $\mathrm{L}$ for smaller EPA.

Theoretical studies ${ }^{(7)}$ indicate that these spectral variations can be explained through a simple model that assumes that the source of these protons is at or near the magnetopause. The protons are assumed to migrate rapidly in L space through the violation of the third adiabatic invariant for trapped particles, but with the preservation of the magnetic moment $\mu$ and line integral I invariants. As the protons drift inwards they are accelerated, the exponential spectrum gets harder, and the value of $\mathrm{E}_{0}$ increases. For EPA $=90^{\circ}$ the field $\mathrm{B} \propto \mathrm{L}^{-3}$, and $E_{0} \propto B \propto \mathrm{L}^{-3}$, in agreement with the experimental data (see Figure 24).

Other studies indicate that L-space motion through geomagnetic field changes such as sudden commencements and sudden impulses may be adequate to explain the variations in fluxes with $L$ when loss processes are also included. ${ }^{(78)}$

Observations at $\mathrm{L}$ values between 5 and 8 indicate that large time variations do occur for protons with energies greater than $1 \mathrm{Mev}$, but that protons near $100 \mathrm{kev}$ are relatively more stable. As expected, the data at these larger L are 


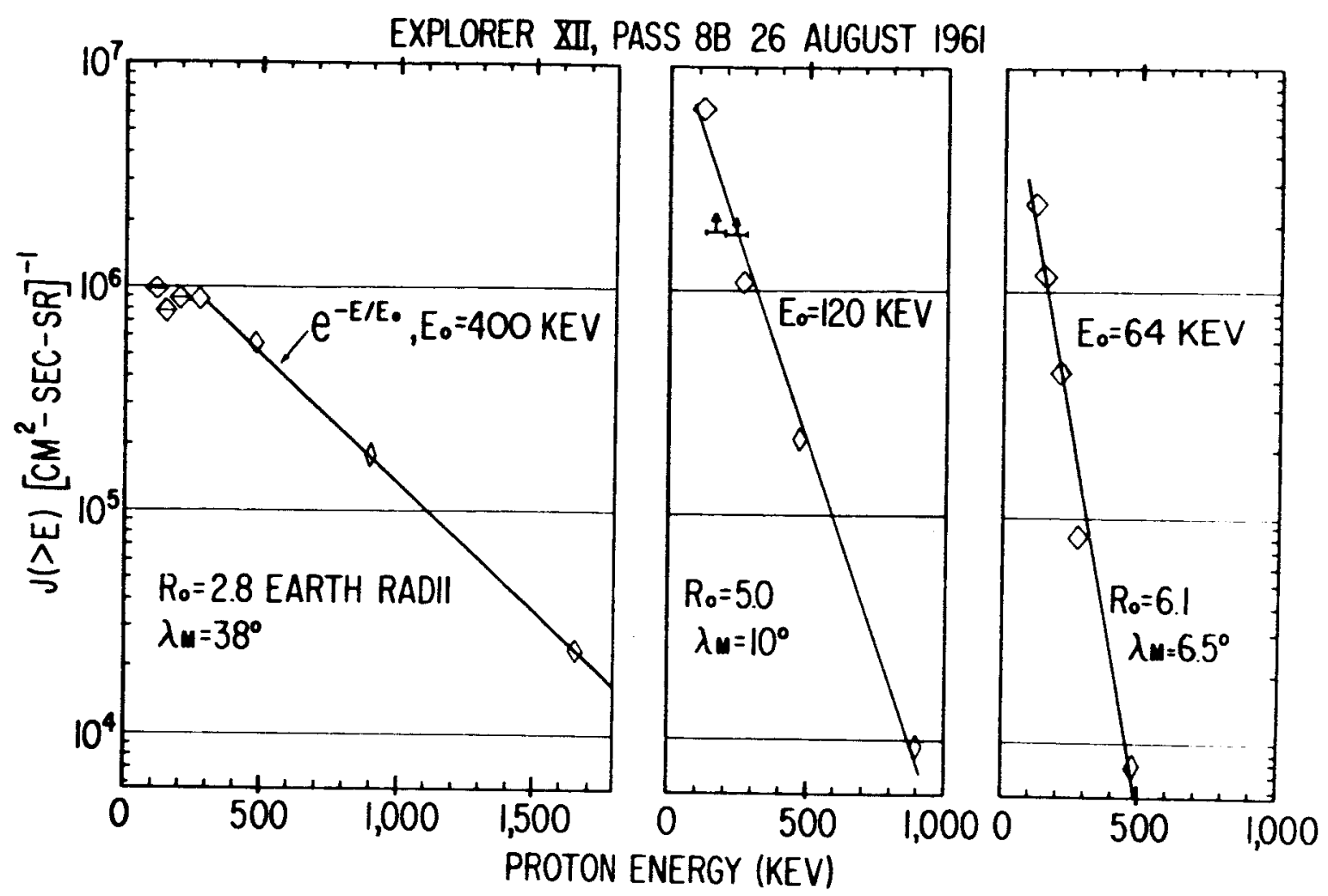

Figure 23- Energy spectra of low-energy protons measured by Explorer XII at several locations in the outer zone of the radiation belt.

not well ordered by magnetic field models that do not take into account perturbing fields such as those produced by the solar wind at the magnetopause.

Because of the large energy density of these outer belt protons, the hope arose that these protons might be the cause of the ring current. However, calculations with measured fluxes indicate that these protons make only about a 10 , disturbance field at the earth's surface. ${ }^{(79)}$

In 1959 a rocket carrying an electron spectrometer showed that the penetrating particles in the outer belt were electrons. ${ }^{(80)}$ An outstanding

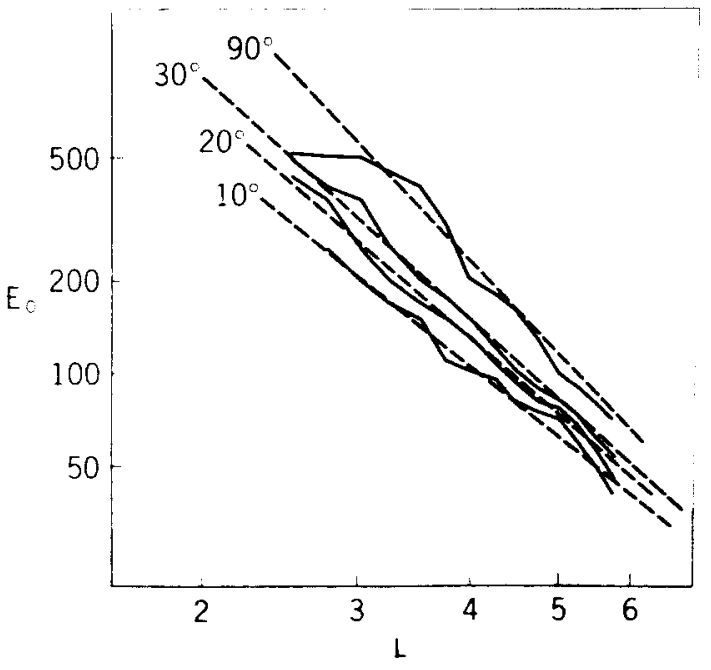

Figure 24-The dotted lines are theoretical curves showing how the e-folding energy $E_{0}$ should vary assuming the outer zone protons move rodially, conserving .. and $\mathrm{I}$. The solid lines ore the experimental values of $E_{0}$ from Explorer XII data. 
difference between electrons and protons in the outer zone is the large (factors of 100) variability in the electron fluxes in times of the order of hours ${ }^{(81)}$ Detectors on Explorer VI showed that electron flux changes were large, especially at the times of magnetic storms. The $E>1.5 \mathrm{Mev}$ electrons at about $\mathrm{L}=3$ frequently decrease, sometimes nearly disappearing, during a large storm, while the low energy $\mathrm{E}>40 \mathrm{Kev}$ flux may increase. Mcllwain ${ }^{(58)}$ showed how the $\mathrm{E}>5 \mathrm{Mev}$ electrons injected into the field by a nuclear explosion behaved during storms (see Figure 25). Several storms decreased the flux at $L \sim 4$, but then in December the flux increased by a factor of about 100. On the basis of Lunik II there are not thought to be any $\mathrm{E}>5 \mathrm{Mev}$ electrons present at quiet

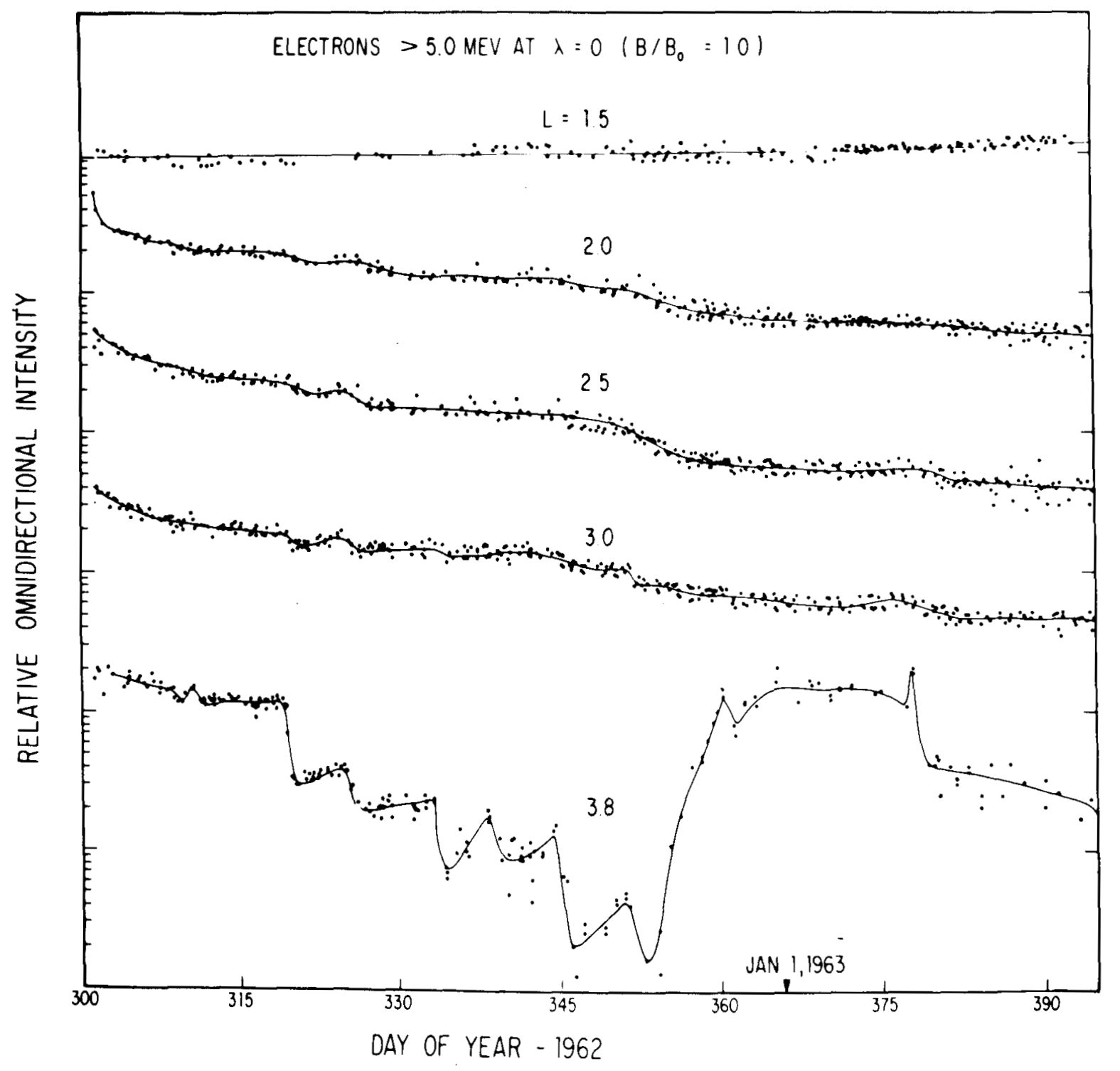

Figure 25-Time variations of $E>5 \mathrm{Mev}$ electrons in the outer zone measured on Explorer $X V$. 
times in the natural belt. ${ }^{(3)}$ This increase therefore is indicative of acceleration of lower energy particles to above $5 \mathrm{Mev}$, although the possibility of injection of $\mathrm{E}>5 \mathrm{Mev}$ particles cannot be excluded. The spectra of electrons also show changes with time. Because of this variability, it is now fairly certain that electrons from albedo neutrons are not an important source of the outer belt.

If typical average values are compared with protons, it is rather surprising that similarities do occur. The electron energies extend to a few Mev, as do proton energies. The spectra show grossly similar fall-off with increasing energy. The softening of spectra with $\mathrm{L}$ is also similar. Fluxes are also comparable, although electron fluxes tend to be lower. Perhaps the similarities point to common mechanisms operating on protons and electrons. In fact, the magnetic field fluctuation mechanis $\mathrm{m}^{(77)}$ that seems to be important for protons was initially developed to explain the electrons in the outer radiation belt.

O'Brien showed on Injun I and III that there were frequently large fluxes of precipitated electrons striking the upper atmosphere in the region of the outer belt. ${ }^{(82,83)}$ At the auroral zone there is always precipitation. He showed that outer belt trapped fluxes increased when precipitation increased, leading to the splash-catcher model of the outer belt. It would seem that there might be a common source for the aurora, precipitated electrons, and outer belt trapped particles.

Van Allen has shown that the outer limit of the radiation belt is not symmetric $^{(84)}$ (see Figure 26). The radial limit of trapping is at about $10 \mathrm{R}_{e}$ at the subsolar point and at about $8 \mathrm{R}_{\mathrm{e}}$ on the back side. This asymmetry agrees with the magnetic field model of Mead, ${ }^{(2)}$ and is just due to the effect of the solar wind on the field. In keeping with this asymmetry a day-night difference in the upper latitude limit of trapping has been observed. ${ }^{(85,86)}$ Bostrom and Williams have found that during magnetically quiet times the day-night difference is about $2^{\circ}$, as expected from Mead's model, but for disturbed times the difference is larger. ${ }^{(87)}$

\section{E. Other Belts}

We know something about radiation belts on some other planets. Decimeter radio radiation from Jupiter ${ }^{(88)}$ has been identified as being synchrotron radiation from trapped electrons. ${ }^{(89)}$ It shows linear polarization and the radio source is more than three times the width of the planet; ${ }^{(90)}$ both facts are in keeping with a radiation belt source. If the surface magnetic field is about 10 gauss, as is suggested from other Jupiter radio waves, then the belt should consist of about $10^{8}$ electrons $/ \mathrm{cm}^{2}-\mathrm{sec}$ of $\mathrm{E}>10 \mathrm{Mev}$ at about $2 \mathrm{R}_{\mathrm{Ju}}$ to give the observed synchrotron radiation. ${ }^{(91)}$ This is a very intense electron belt compared to the 


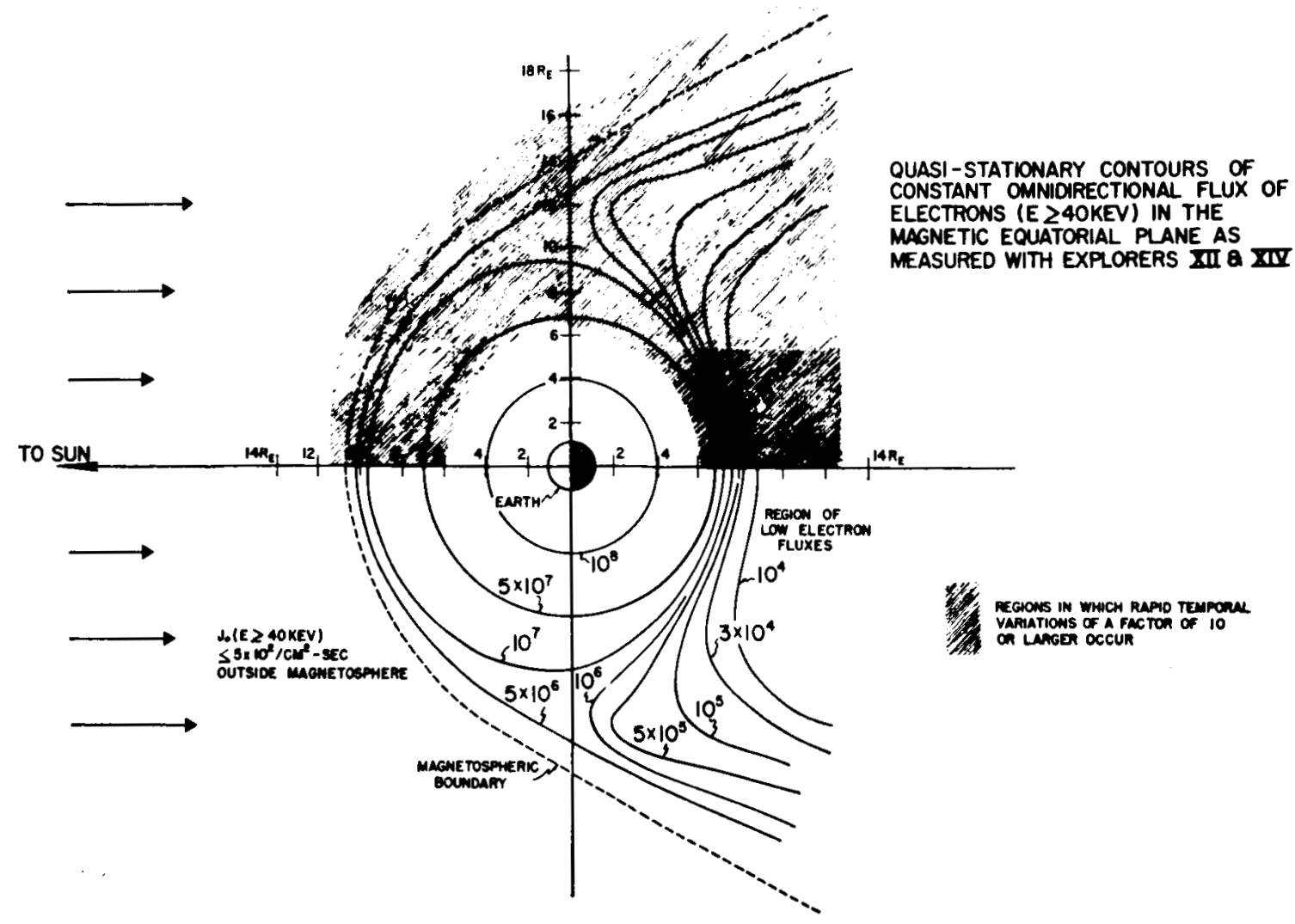

Figure 26-Flux contours of $\mathrm{E}>40 \mathrm{Kev}$ electrons in the outer radiation belt showing the day-night asymmetry.

earth's. The synchrotron radiation from the terrestrial natural Van Allen belt is too low to be measured. Synchrotron radiation however was measured from the artificial belt formed by the Starfish event.

The Mariner satellite passed about $40,000 \mathrm{~km}$ away from Venus on the sunward side. At this distance there was no evidence for a planetary magnetic field ${ }^{(12)}$ or any evidence of trapped particles. ${ }^{(92,93)}$ This does not eliminate the possibility of a radiation belt, but only means that Mariner stayed outside the Venusian magnetosphere and that therefore, the Venus surface field can be no greater than about $10 \%$ of the earth's.

There is no evidence of the existence of any other radiation belts. Lunik II showed that the moon's field is less than $100 \%$, so that it can hardly have a radiation belt..$^{(9)}$ No other planets show significant synchrotron radiation.

F. The Aurora

Despite the many fine experiments that have been performed in the satellite era for the understanding of auroras, their explanation remains elusive. The 
elimination of theories cannot usually be claimed as triumphs; but the virtually complete elimination of two such seemingly obvious ones rank as significant accomplishment.

The old one of these theories, due to Störmer, explained auroras as due to solar particles that entered the earth's magnetic field directly near the auroral zones. ${ }^{(39)}$ Both Russian ${ }^{(95)}$ and U.S. deep space probes ${ }^{(06)}$ have shown that sufficient fluxes of such energetic solar particles do not exist in the interplanetary medium away from the earth.

The other unsuccessful auroral theory was born as recently as the satellite era. Early low-altitude satellites detected large fluxes of electrons with sufficient particle energies to contribute to the production of auroras. The hope arose that fluxes large enough to explain auroras might exist at higher altitudes; then, some mechanism for the dumping of the trapped electrons from the Van Allen belts was all that would be required for the explanation of auroras. However, measurements both by Russian ${ }^{(97)}$ and U.S. scientists ${ }^{(98)}$ indicated that although the higher altitude fluxes were larger, they were insufficient to account for auroras. A flux tube of the Van Allen belt would be drained in a matter of seconds by a strong aurora; but such an aurora can last hours. A most significant finding by $\mathrm{O}^{\prime} \mathrm{Brien}^{(82)}$ was the discovery on Injun of the increase in the trapped particle population when large fluxes of electrons entered the atmosphere (See Figure 27). Thus the elimination of the auroral theory of the dumping of trapped particles has given rise to the view that whatever causes auroras is also a major contributor to radiation belt population.

Beside these results that have eliminated theories, many discoveries which limit new theories have been made. O'Brien showed that there were electron fluxes of at least $10^{4} \mathrm{~cm}^{-2} \mathrm{sec}^{-1}$ being lost all the time in the auroral zone (See Figure 28), and that when this flux increased, visual aurorae occurred.

The particles which contribute most to auroral emission have been found to be electrons with energies below $25 \mathrm{Kev}$. However, electrons with energies above $25 \mathrm{Kev}$ and with energies as high as $100 \mathrm{Kev}$ are also associated with auroras. Most of the auroral light seems to be produced by electrons near $10 \mathrm{Kev}$. Certain auroras are excited by both electrons and protons, and others appear to be excited predominantly by either electrons or protons.

McIlwain, ${ }^{(99)}$ Davis, ${ }^{(100)}$ and McDiarmid ${ }^{(111)}$ have flown rockets into active auroral displays, and have found that electrons of a few Kev are the commonlyfound particles. The narrowness of some auroral arcs agrees with electrons being the active particles-the proton cyclotron radius would be larger. The common altitude of visual auroras of $100 \mathrm{~km}$ is what is expected for electrons 

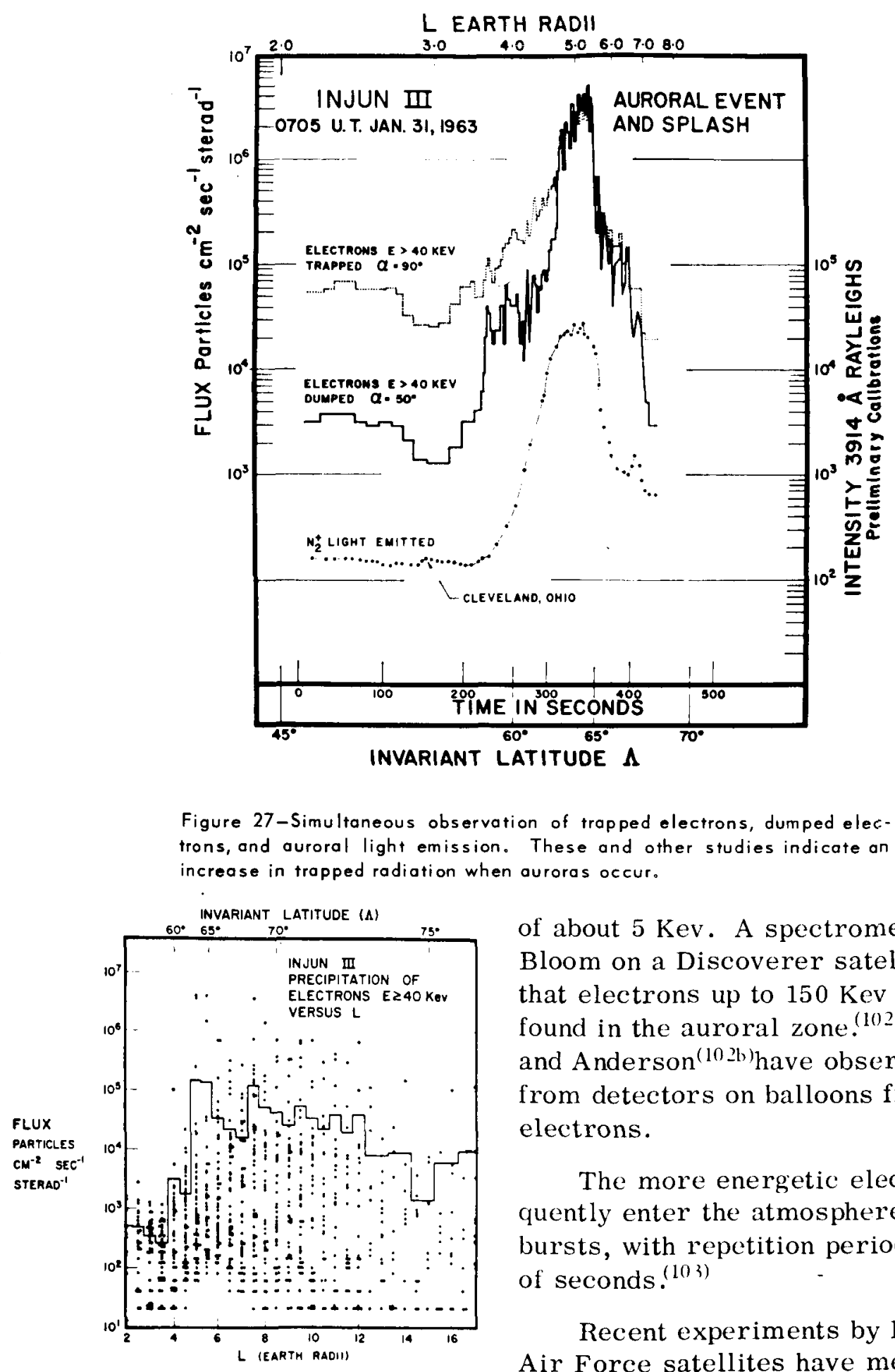

Figure 28-Sample of precipitated flux. Note that near $L=6$ some precipitated flux is observed at all times since all observations show more than background fluxes.

of about $5 \mathrm{Kev}$. A spectrometer flown by Bloom on a Discoverer satellite showed that electrons up to $150 \mathrm{Kev}$ are frequently found in the auroral zone. (102) Brown $^{(102 a)}$ and Anderson ${ }^{(102)}$ have observed $\mathrm{x}$-rays from detectors on balloons from such electrons.

The more energetic electrons frequently enter the atmosphere in short bursts, with repetition period of the order of seconds. (103)

Recent experiments by Evans ${ }^{(104)}$ on Air Force satellites have measured the electron energies down to $80 \mathrm{ev}$. They show that there are usually fewer electrons at these energies than at $1 \mathrm{Kev}$. 
Both magnetic field and trapped particle measurements have made it possible to follow field lines from where auroras occur. These studies show that many auroras occur on field lines that contain trapped particles and that connect directly to the opposite hemisphere. However, many auroras appear to occur near the outer limit of trapping field lines.

Satellite measurements of vlf energies in the magnetosphere at the time of auroras and on the same field lines indicate that vif energies are insufficient to be the producers of auroral particles and that if there is a causal relationship, what produces auroras also produces vlf.$^{(105)}$

Satellites have been equipped with sensitive optical sensors to observe auroras from above.(106) Although these satellites do not stay in the auroral regions very long, they have the advantage of no cloud interference and the ability to scan large areas. These sensors have shown that over some $5^{\circ}$ of latitude over the auroral zones, auroras were detected at all times; in the $5^{\circ}$ latitude strips bordering the main auroral zone, auroral emissions were detected a large fraction of the time.

Because of the elimination of several lines of approach, promising areas for experimentation and study are reasonably obvious and include phenomena in the tail of the magnetosphere and electric fields. As yet there is now no satisfactory explanation of the source of aurorae. 
IV.

\section{THE EARTH'S MAGNETIC FIELD}

An accurate knowledge of the earth's magnetic field is desirable for many reasons. Reliable charts have, of course, always been needed for nautical and aeronautical navigation. Also, a detailed knowledge of the main field and its time history are a necessary prelude to a fundamental understanding of the origin of the field and its secular variations. In addition, the space age has brought with it its own peculiar needs, particularly in describing the region between the earth's surface and the magnetospheric boundary. First of all, an accurate main field reference is needed for rocket and satellite magnetic field measurements designed to locate and identify current sources external to the earth. Secondly, the motion and distribution of trapped particles and the trajectories of solar particles and cosmic rays as they approach the earth are determined by the earth's magnetic field. Finally, knowledge of the detailed geometry of field lines is necessary for locating conjugate points, for ionospheric research, and for understanding aurorae, whistlers, and other geomagneticallycontrolled phenomena.

At the present time, the accuracy of field descriptions are limited by the availability of up-to-date world-wide magnetic data. However, the density of available data over the earth's surface is extremely non-uniform. More than enough data is available over North America and Europe, for example, but data is essentially non-existent over vast parts of the Pacific Ocean and in the Antarctic region. In addition, whatever data that does exist in some of these poorlymapped regions were obtained, in many cases, more than 50 years ago. The secular changes in the earth's field which are continually taking place make much of this data useless for describing the field as it now exists. And finally, the older mathematical techniques required that values of the field be taken at regular grid intervals in order to determine the spherical harmonic coefficients for a mathematical description of the earth's field, thus the basic data itself could not be used, but one had to depend upon the ability of the chart-maker to draw accurate contours and determine the values at the grid intervals. In the past, two or more workers which began with essentially the same data have arrived at sets of coefficients which differ significantly from one another.

Much progress has been made on the last of these problems. The availability of high-speed computers has made it possible to use raw data directly in determining coefficients, thus eliminating the intermediate contour maps. 
Much of the data has been digitized and transferred onto cards and tape, so as to make it usable for computers.

Satellites appear to be particularly suitable as a basic source of up-to-date world-wide magnetic data. All parts of the world are accessible to a polarorbiting satellite, and extensive data can be obtained over a short period in which secular changes are negligible. So far, however, there has not been a truly world-wide survey of this type. The Vanguard 3 satellite, launched in September, 1959, made measurements of the total field at altitudes of 510 to $3753 \mathrm{~km}$ over certain sections of North and South America, Australia, and Southern Africa. ${ }^{(107)}$ Since these regions covered only a small fraction of the earth's surface, the spherical harmonic coefficients which were obtained as a best fit to the data were not judged to be useful as an accurate description of the entire earth's field. However, the data have been included along with other ground-based data in obtaining the Jensen and Cain coefficients for epoch $1960 .{ }^{(76)}$ Some magnetic data over North America have also been obtained from the Canadian Alouette Satellite, by analyzing the ionograms for multiples of the electron cyclotron frequency. The next major satellite project for accurate determination of the earth's field is an experiment on POGO, which is to be launched late in 1965 into a polar orbit. ${ }^{(108)}$ It is hoped that data from this experiment will increase the accuracy with which the earth's field is described by almost an order of magnitude.

The World Magnetic Survey (WMS), ${ }^{(108)}$ a cooperative effort involving personnel and equipment from over 30 nations, will produce much additional groundbased and satellite data, concentrating on the present period of the quiet sun. As part of this effort, data is presently being obtained by the Russian non-magnetic ship, the Zarya, in the Atlantic, Pacific, and Indian Oceans, and by Project Magnet, a U.S. effort in which a specially-equipped airplane flies over selected land and sea masses. Established magnetic observatories (the U.S. operates 11) continue to provide up-to-date data for the regions in which they are located.

Due to the distortion of the earth's field by the solar wind and the presence of charged particles in the magnetosphere, the ordinary spherical harmonic expansion of the earth's field is no longer accurate at large distances from the earth. Explorer $6,^{(14)} 10,,^{(32)} 12,,^{(18)} 14,,^{(109)}$ and 18 (IMP-1) ${ }^{(13)}$ all contained magnetometers which observed these effects. In general, they have found that the measured field is usually stronger in the equatorial regions than that which one would expect on the basis of a simple extension of the earth's internal field. In addition, the direction of the field is often significantly altered. As a result, field lines emerging from the earth at high geomagnetic latitudes become grossly distorted. The effects depend strongly on the relative position of the sun, indicating the importance of the solar wind. Since there are large temporal changes 
in the strength of the solar wind and the distribution of charged particles in the magnetosphere, it is difficult to obtain an accurate mathematical description of the field at every point in space. So far, most of our understanding of the topology of the field in the magnetosphere is qualitative in nature. Our present picture, however, is vastly different from that which was generally accepted six or eight years ago, before information from satellites and space probes forced us to re-examine the basic concepts. The effects of the solar wind on the earth's magnetic field were considered in greater detail in Section $\Pi$ of this paper.

Much effort has been expended in exploring and attempting to understand magnetic field variations. Many types of variations are closely related to solar activity and must therefore be caused, directly or indirectly, by corpuscular or electromagnetic emissions from the sun. A magnetic storm, for example, typically has several clearly-distinguishable phases, which are most clearly seen in the records of the horizontal component of the earth's field. The sudden increase in the magnitude of this component, referred to as a sudden commencement, is now known to be due to the arrival at the earth of an additional burst of plasma from the sun. This provides an additional compression of the magnetosphere, thus increasing the field. This increase usually lasts for several hours (the initial phase), and is generally followed by a much larger decrease in the horizontal component, lasting for several days (main phase). This decrease is still not well understood.

Singer postulated that the main phase was caused by the longitudinal drift of trapped particles in the earth's field, producing effectively a ring current. ${ }^{\left({ }^{(0)}\right.}$ The whole question of ring currents and the main phase of magnetic storms has been reviewed recently by Akasofu ${ }^{(110)}$ and Smith.(111) Attempts have been made with Explorer 6 data ${ }^{(12)}$ and Explorer 10 data $^{(32)}$ to relate magnetometer measurements with possible ring currents. Up to the present time, however, no satellite magnetometer data have definitely located the position of the postulated ring current. None of the trapped particles fluxes which have been observed by satellites, either electrons or protons, are intense enough to produce the observed main phase decrease. The low-energy protons observed by Davis and Williamson ${ }^{(75)}$ produce only about $10 \gamma$ reduction, ${ }^{(79)}$ much less than that actually observed during storms. The existence and location of the ring current is still a major unsolved question.

Often the horizontal component is observed to increase suddenly, but without a subsequent main phase. Such increases are called sudden impulses if they are observed world-wide. Nishida and Cahill have shown these impulses to be related to similar magnetic changes observed by Explorer 12 at large distances from the earth, and have related them to shifts in the magnetopause boundary 
due to increased solar wind pressure. ${ }^{(113)}$ Further evidence for the correlation of solar wind properties with magnetic activity comes from the Mariner 2 space probe, ${ }^{(8)}$ where it has been shown that the velocity of the solar wind is strongly correlated with the $k_{p}$ index (an index of magnetic activity in midlatitudes). 


\section{SOLAR ENERGETIC PARTICLES AND GROUND EFFECTS}

Before satellites, measurements had been made that indicated that energetic particles occasionally arrive at the earth from the sun. Even before the IGY, sea-level cosmic ray neutron monitors had observed a few events. ${ }^{(114)}$ In February 1956 a rare white light flare occurred that produced protons of over one Bev, so that they could be detected by sea-level neutron monitors. (115)

The first direct evidence that the sun produces large fluxes of low-energy protons in the energy range around several hundred Mev was obtained by chance on March 26, 1958, when a balloon was flown by Winckler's group at Minneapolis during the main phase of a moderate geomagnetic storm.116) Counters on the balloon showed a steadily increasing count rate during the flight. Nuclear emulsions recovered from the flight showed large numbers of protons of $\mathrm{E} \sim 100 \mathrm{Mev}$. A large east-limb flare on March 23, 1958 had produced a radio blackout in the polar regions. This blackout was caused by absorption in the D layer of the ionosphere due to increased ionization produced by the incident protons. Subsequent to this event riometers, which measure the absorption of galactic radio noise in the ionosphere, have been used to give warnings that solar protons are incident on the polar atmosphere. Several of the polar cap absorption (PCA) events have been studied by launching balloons and rockets when PCA events were detected. Charakhchian in the U.S.S.R. also studied solar proton events since July 1958 by twice daily routine balloon flights. ${ }^{(117)}$ Winckler, ${ }^{(118)}$ by studying the ascent and descent records of balloons, assuming an isotropic incident proton flux and correcting for atmospheric loss, was able to deduce an energy spectrum for the solar protons. He also showed that geomagnetic cutoffs were changed during some solar proton events at the time when the magnetic storm started on earth; this was some time after the start of the PCA event. Protons down to $80 \mathrm{Mev}$ or even less have been found at Minneapolis, where normally the cutoff is about $700 \mathrm{Mev}$. Obviously the magnetic field must be considerably disturbed at this time.

Emulsions flown on balloons have been used to measure proton energy spectra; they have also shown that besides protons there were : particles present in the energetic solar particles. The $\alpha / p$ ratios vary widely from $1: 1$ to $1: 30$ for different events. Emulsions flown by Fichtel ${ }^{\left(11^{19)}\right.}$ on sounding rockets from Fort Churchill during the September 3, 1960, and November 12 and 15, 1960, PCA events gave data on the charge composition of the solar protons up to $\mathrm{z}=8$ relative to carbon as shown in the following table. 
$\underline{\text { Relative Abundances }}$

$$
\begin{array}{lllll}
\mathbf{P} & \mathrm{Be}, \mathrm{B} & \mathrm{C} & \mathrm{N} & 0
\end{array}
$$

Galactic cosmic rays (same

rigidity interval)

Sun

$\begin{array}{ccccc}2.6 \times 10^{3} & 5 & 10 & 5 & 6 \\ 20 \times 10^{3} & 10^{-5} & 10 & 2 & 18\end{array}$

Solar cosmic rays (same rigidity interval)

$(2.6-50) \times 10^{3}$

$0.5 \quad 10 \quad 6$

19

The interesting observation here of a relatively high abundance of $\mathrm{B}$ and $\mathrm{Be}$ shows a considerable difference from solar composition. These elements enter into thermonuclear reactions and are easily burned up. Any proposed source mechanism must explain the existence of these light elements.

Satellite observations of solar protons ${ }^{(44)}$ started with Explorer IV in August 1958. Detectors on Explorer VII ${ }^{(120)}$ observed several events in 1960 and Injun I studied events in July 1961.(121) By using data from neutron monitors, counters on satellites, balloons, rockets and riometers, rough energy spectra and time histories of events from 1958 to 1961 have been constructed. ${ }^{(122)}$ Instruments flown by McDonald's group on Explorer XII allowed a detailed energy spectrum as a function of time to be measured for the first time for most of the September 28, 1961, solar proton event. ${ }^{(123)}$ Because Explorer XII was part of the time outside the geomagnetic field it could give unequivocal data of proton fluxes down to about $3 \mathrm{Mev}$. These measured flux-time histories have provided information about the mode of propagation of the particles from the sun. If they did, they would arrive at the earth from preferred directions. This is not the usual situation; the solar protons arrive generally isotropic at the earth. They diffuse to the earth as can be demonstrated directly from the experimental data. Isotropic diffusion from a point source gives a flux distribution which should obey a law of the form

$$
J(r, t)=\frac{\text { const }}{t^{3 / 2}} e^{3 r^{2 / 4 v \lambda t}}
$$

A test of this law is shown in Figure 29 which shows that after an initial period of an hour or so isotropic diffusion seems to apply. ${ }^{(124)}$ This is really too simple a model. The presence of the magnetic field means the diffusion cannot be really 
isotropic. The solar magnetic field is apparently disturbed enough so that the protons are frequently scattered with an effective mean free path of about .04 AU. The diffusion problem can be worked backwards to determine the source energy spectrum at the sun. This source spectrum can be described by a power law in kinetic energy from a few Mev to several hundred Mev. ${ }^{\text {(24) }}$

Another interesting observation made on Explorers XII and XIV is that there are periodic fluctuations in intensity that are simultaneous at all energies, with periods the order of one hour. These seem to be local, i.e., close to the magnetopause, because they happen at all energies together and suggest periodic oscillations of some type in the transition zone..$^{(125)}$

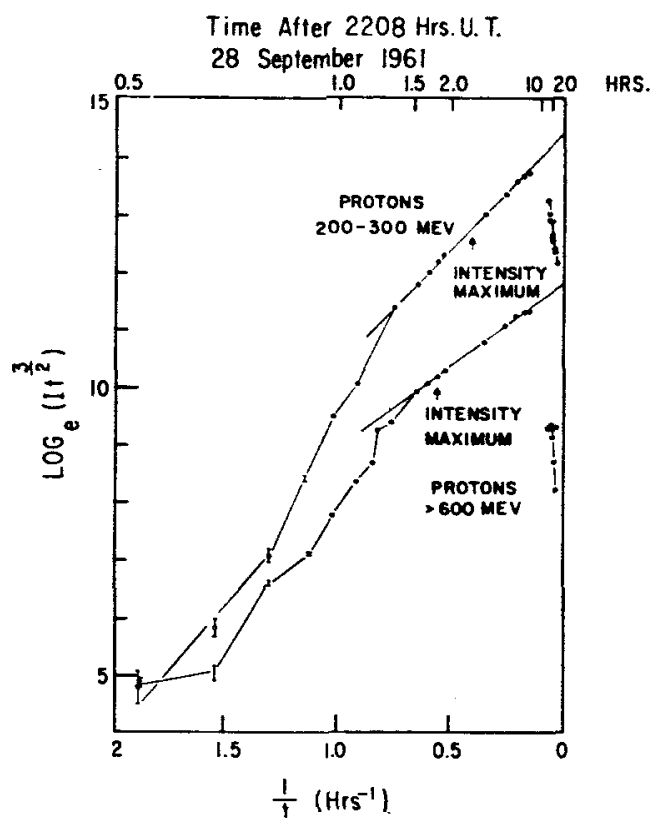

Figure 29-A Test of diffusion theory opplied to solar protons. After about one hour the test works.

The high energy solar protons that arrive early in an event do sometimes come preferentially from about $45^{\circ}$ west of the sun.(125) This is what is expected if the particles are guided by the spiral solar magnetic field. This directionality doesn't last long and is present only for events that occur near the west limb of the sun. The west limb is rather well connected magnetically to the earth due to the spiral-shaped solar field. Protons from east limb flares take longer to get to the earth and are isotropic, probably because the particles have to diffuse across the magnetic field. A sketch of the history of a solar proton event ${ }^{(122)}$ is shown in Figure 30.

Data from Explorer XII by McDonald ${ }^{(126)}$ and from Explorer 18 (IMP-1) by Simpson have shown that certain solar proton events return with a 27 -day periodthe rotation period of the sun (see Figure 31 ). This very interesting observation suggests that certain magnetic field configurations periodically engulf the earth as the sun rotates, and that there is either energetic-particle production or particle storage for a month or more (see Figure 1).

We know relatively little about the mechanism of production of energetic solar protons. It seems that the energy for solar flares must come from converting magnetic field energy to particle energy and light. This may occur in an electrical discharge at a magnetic null point. ${ }^{(127,128)}$ Severny has observed that the magnetic field before and after a flare are different, in agreement with 


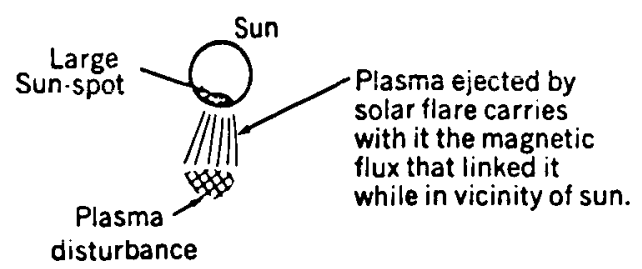

EÅTH

(a) 6 hours ofter flare

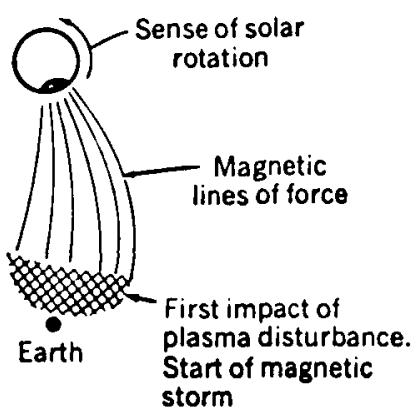

(b) About 24 hours after flare

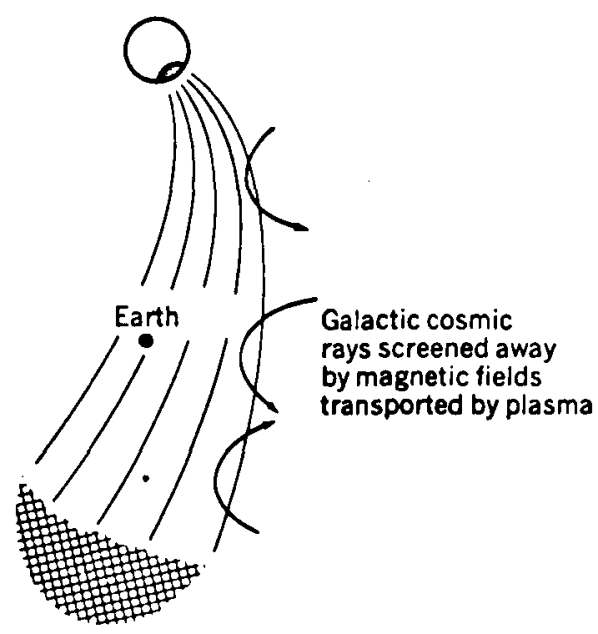

(c) About 48 hours after flare

Figure 30-History of a solar proton event. this idea. ${ }^{(129)}$ While the picture appears to be reasonable, there is no satisfactory quantitative theory of the process..$^{(130)}$

Detectors on IMP-1 and EGO have not observed any large solar proton events during the last year. As might be expected, there appear to be few such events near solar minimum.

The solar proton events are important not only in themselves but in that they constitute a radiation hazard for space flight. Webber has summarized the energy spectra from several events (see Figure 32) and the doses that would have been received under different shield thicknesses from the large events of the last solar cycle.

Under $1 \mathrm{gm} / \mathrm{cm}^{2}$ the three July 1959 events produced $885 \mathrm{rad}$. Because these events are so large and infrequent, it is of interest to try to predict them in advance. Without any fundamental understanding of the origin of the energetic particles, empirical schemes have been tried. Various ideas involving the area, magnetic character and number of solar rotations of the sunspot groups have been used.(132) The best prediction system may be that of Severny, who uses a measure of the gradient of the magnetic field as an index of probable occurrence. 


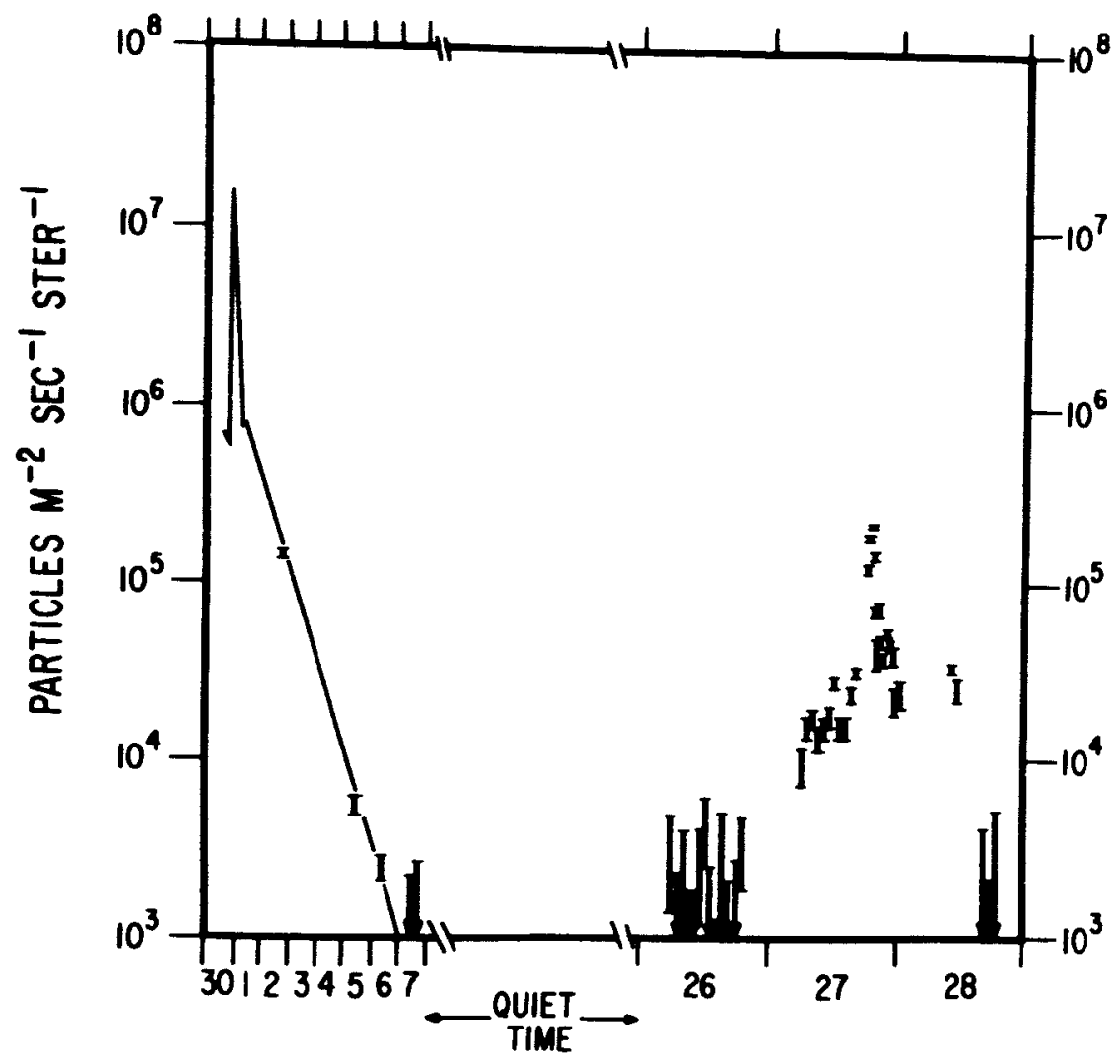

OCTOBER 196|

Figure 31-A recurrent solar proton event measured on Explorer XII in October, 1961.

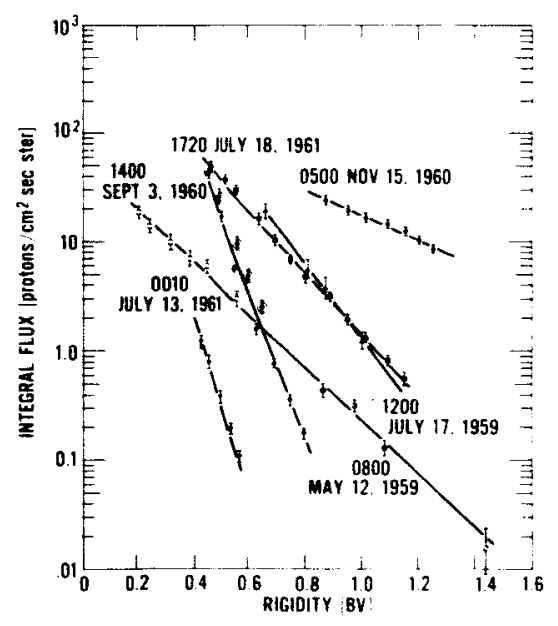

Figure 32-Proton energy s pectra from several solor proton events. 
VI.

\section{COSMIC RAYS}

In the last 20 years it has been well established that the sun occasionally emits energetic particles (erroneuously called cosmic rays). Here we will deal with galactic cosmic rays coming to the earth from outside the solar system. The cosmic rays observed at sea level are essentially all secondaries produced by the interaction of primary cosmic rays with the earth's atmosphere. Mesons resulting from these interactions were first identified in cosmic ray emulsion experiments before they were made in laboratory accelerators. To study the primary cosmic rays it is necessary to get above most, if not all of the atmosphere. Studies on balloons have shown that primary cosmic rays are about $94 \%$ $\mathrm{H}, 5 \% \mathrm{He}$ and $1 / 2 \%$ heavier nuclei. Considerable effort has been devoted recently with balloon measurements to get the composition of the heavy nuclei. In these studies care must be taken to correct for the fragmentation of the incoming nuclei by the few $\mathrm{gm} / \mathrm{cm}^{2}$ of air above the balloon. The results of this work are ${ }^{(133)}$ summarized below

\begin{tabular}{ccc}
$\begin{array}{c}\text { Nuclear } \\
\text { Charge }\end{array}$ & $\begin{array}{c}\text { Particles/ } \\
\mathrm{cm}^{2}-\mathrm{sec}-\text { ster } \\
\text { of } \mathrm{E}>2.4 \mathrm{Bev} / \text { nucleon }\end{array}$ & $\begin{array}{c}\text { Ratio of CR Abundance/ } \\
\text { Cosmic Abundance } \\
(\mathrm{H}=1)\end{array}$ \\
\cline { 2 - 2 } $3<\mathrm{Z}<5$ & 2.0 & very large \\
$6<\mathrm{Z}<9$ & 5.6 & 2.5 \\
$10<\mathrm{Z}<19$ & 1.88 & 9.0 \\
$20<\mathrm{Z}<28$ & 0.69 & 63.0
\end{tabular}

The relatively large number of $3<z \cdot 5$ is interesting. These nuclei enter into thermonuclear reactions easily and are burned. Therefore, they are rare in stellar atmospheres. There is also an interesting excess of heavy cosmic ray nuclei. This must be explained by any satisfactory theory of the origin of cosmic rays. Recent work by Earl, (134) Meyer ${ }^{(135)}$ and others has identified electrons in the primary radiation. The $\mathrm{e} / \mathrm{p}$ ratio is only about $1 \%$. So the electron flux is not very large. McDonald on Explorer 18 (IMP-1) has found 3to $12-\mathrm{Mev}$ electrons in space.(136) These may be of galactic orgin. There are two models of production of the electrons: (a) collision of a cosmic ray proton and an atom to generate $\pi$ mesons which decay to electrons, and (b) acceleration of low energy electrons by something like the Fermi process. A recent 
measurement of the electron-positron ratio of $\mathrm{e}^{+} / \mathrm{e}^{-\sim 0.3}$ helps confuse this problem. ${ }^{(137)}$ From acceleration essentially no $\mathrm{e}^{+}$are expected. From collisions ${ }^{(138)} \mathrm{e}^{+} / \mathrm{e}^{->} 1$ so the experiment seems to say both sources are required, but this matter cannot be considered settled.

Simpson and McDonald ${ }^{(139)}$ on IM P-1 have measured the low energy proton and alpha energy spectra well away from the earth's field. Ground-based studies of extensive air showers of the past few years have shown that cosmic rays up to $10^{19} \mathrm{ev}$ exist. It seems improbable that these particles can be stored in the galaxy; the radius of curvature is toolarge. We have the probability then that there are intergalactic cosmic rays. Parker pointed out the possibility that if the cosmic ray flux outside the solar system is about ten times that at the earth, the energy density in cosmic rays should exceed the energy density in magnetic fields there, and the cosmic rays then should freely flow into intergalactic space. This idea that intergalactic space is filled with cosmic rays is a novel one and would pose a problem for cosmologists. A measurement of the cosmic ray gradient away from the sun would help answer this problem.

On Pioneer $V$ Simpson ${ }^{(1.40)}$ observed a Forbush decrease in cosmic rays a long way from earth, demonstrating that the Forbush decrease process operating on cosmic rays is not geocentric but heliocentric, and quite obviously associated with a blast of strong solar wind being emitted. 
VII.

\section{NEUTRONS}

The neutron flux in space produced by galactic cosmic rays interacting with the earth's atmosphere is of interest not only in itself, but because we know that the decay of neutrons is an important source of inner-zone trapped protons and probably also electrons. This neutron flux was measured on an Atlas rocket by Hess, ${ }^{(14)}$ and more recently on several satellites by Lockwood, (142) Martin, ${ }^{(143)}$ and Bostrom and Williams. ${ }^{(14)}$ It is probably known for quiet times to about 50 percent accuracy and is

$$
\text { Neutrons } / \mathrm{cm}^{2}-\mathrm{sec}
$$

0.2

1.5

\section{$\underline{\text { Latitude }}$}

0

$90^{\circ}$

The energy spectrum of the neutrons has not been measured in space, but it has been measured in the atmosphere ${ }^{(1.15)}$ and calculated for free space. ${ }^{(16)} \mathrm{Cal}-$ culations using this data show quantitative agreement with the measured innerzone protons. There seems no reason to doubt that neutron decay produces the energetic inner-zone protons. Galactic cosmic rays colliding with atmospheric 0 or $\mathrm{N}$ nuclei are the source of these neutrons. There are other sources of neutrons. Solar protons made in connection with solar flares undoubtedly produce neutrons when they strike the polar atmosphere. ${ }^{(1 /)}$ It is commonly thought that these neutrons produce the low energy proton component observed by Naugle and Kniffen ${ }^{(56)}$ for $L>1.7$ on the NERV rocket, but no direct measurements of these solar proton produced neutrons have been made yet.

The sun is also probably an important source of neutrons. Measurements by Hess ${ }^{(148)}$ on OSO of the diurnal variation of neutrons observed near the earth showed that less than 1 percent of them can be of solar origin. Solar flares should be able to produce neutrons but no measurements have detected them yet. 
VIII.

\section{CONCLUSIONS}

On the basis of the discoveries made in recent years, a new picture has emerged of our immediate environment, and more generally of processes in the solar system. Plasma, both thermal and superthermal, plays a dominant role in our new understanding. Interactions between the magnetic field and charged particles in the plasma of the outer sun are believed to be responsible for the solar activity such as is manifested by sun spots and solar flares. These in turn govern many aspects of the earth-sun relations, partially through electromagnetic radiation such as the solar ultraviolet or $\mathrm{x}$-radiation and partially through the solar wind plasma.

Plasma properties near the sun are determined primarily by the magnetic field. As the coronal plasma expands into the solar wind, the kinetic energy in the particles assumes a dominant role. The magnetic field, however, continues to act as the coupling agent between individual charged particles and produces collective motions. Of great interest for future explorations will be the transition region between these two domains, the azimuthal asymmetries of the solar wind in the plane of the ecliptic, and the character of the plasma outside the plane of the ecliptic.

The sun on occasions produces large soiar flares, some of which emit energetic protons into space. The particles are guided by and diffused along and across the magnetic field to reach the earth. Flares also produce enhanced solar wind fluxes, which reach the earth a day or so later, producing magnetic storms.

The interaction mechanisms of a collisionless plasma such as the solar wind with a large object such as the magnetosphere present in themselves challenging scientific problems. In the case of the earth, this interaction produces a collisionless shock wave and is apparently responsible for certain geomagnetic disturbances, the auroral displays and the outer part of the Van Allen belts. On the basis of the investigations conducted so far, we can now appreciate some of the qualitative aspects of this phenomenon, but a quantitative theory will have to wait for the results from future work. The interaction of the solar wind with the moon and other planets exhibits many different aspects of plasma physics. For instance any magnetic field the moon may have is less than $1 / 200$ of that of the earth, and there are probably periods when the solar 
wind penetrates to the lunar surface. If so, this may alter the optical properties of the lunar surface, and x-rays may be produced in the process.

The inner part of the Van Allen radiation belt is apparently populated mostly by protons and electrons made from the decay of neutrons that have been made in the atmosphere of the earth by galactic cosmic rays. The outer parts of the radiation belts may be thought of as a superthermal plasma in which the magnetic field is the dominant factor. We find here primarily electrons and protons with energies between $10 \mathrm{ev}$ and several hundred kev. Collective interaction of the individual charges with the terrestrial magnetic field permit various modes of hydromagnetic waves to propagate through this region. A special example of these waves are the well-know whistlers. It is believed that these waves, by interaction with the plasma, determine in turn the energy spectrum and lifetime of the energetic charged particles in the outer belts and in the geomagnetic tail. Indications are that at least part of the waves are generated at the interface between the solar wind and magnetosphere and then travel not only through the trapping region, but also through the tail of the magnetosphere.

We are reasonably sure that Jupiter has a radiation belt as well as the earth. Future space exploration will probably show that some other planets do too.

The solar wind extends out from the sun considerably farther than the earth. It impedes flow of galactic cosmic rays inwards into the solar system, and the variability of flow of the wind produces the Forbush decreases and the $11-y e a r$ solar cycle variation of cosmic rays.

One of the most fundamental questions to be answered by future exploration of interplanetary space is how many galactic cosmic rays exist in interstellar space. It is now believed that these cosmic rays play a significant role in the dynamics of the universe, and a knowledge of their flux is required before a realistic cosmological model can be constructed. Intergalactic probes, going beyond the orbit of Jupiter, will be required to obtain answers to this question. Such an investigation will be technically feasible during the next decade. 


\section{BIBLIOGRAPHY}

1. Biermann, L., Kometenschweife und solare korpuskularstrahlung, $Z$. Astrophys. 29, 274-286, 1951.

2. Parker, E. N., Dynamics of the interplanetary gas and magnetic fields, Astrophys. J. $128,664-676,1958$.

3. Parker, E. N., The hydrodynamic theory of solar corpuscular radiation and stellar winds, Astrophys. J. 132, 821-866, 1960.

4. Gringauz, K. I., V. V. Bezrukikh, V. D. Ozerov, and R. E. Rybchinskii, A study of the interplanetary ionized gas, high-energy electrons, and corpuscular radiation from the sun by means of the three electrode trap for charged particles on the second Soviet cosmic rocket, Dokl. Akad. Nauk. SSSR 131, 1301, Soviet Physics Dokl. 5, 361-364, 1960.

5. Bonetti, A., H. S. Bridge, A. J. Lazarus, B. Rossi, and F. Scherb, Explorer 10 plasma measurements, J. Geophys. Res. 68, 4017-4063, 1963.

6. Neugebauer, M. and C. W. Snyder, Solar plasma experiment: preliminary Mariner II observations, Science 138, 1095-1097, 1962.

7. Snyder, C. W. and M. Neugebauer, Interplanetary solar wind measurements by Mariner II, Space Research IV, ed. by P. Muller, 89-113, 1964.

8. Snyder, C. W., M. Neugebauer and U. R. Rao, The solar wind velocity and its correlation with cosmic ray variations and with solar and geomagnetic activity, J. Geophys. Res. 68, (24), 6361-6370, 1963.

9. Bridge, H., A. Egidi, A. Lazarus, E. Lyon and L. Jacobson, Preliminary results of plasma measurements on IMP-A, Space Research $V$, to be published.

10. I. G. Bulletin No. 84, Initial results from the first interplanetary monitoring platform (IMP-1), Trans. AGU 45, 501, 1964.

11. Coleman, P. J., Jr., Leverett Davis, Jr., and C. P. Sonett, Steady component of the interplanetary magnetic field: Pioneer V, Phys. Rev. Letters 5, 43-46, 1960.

12. Coleman, P. J., L. Davis, E. J. Smith and C. P. Sonett, The mission of Mariner 2 preliminary observations-interplanetary magnetic fields, Science 138, 1099, 1962.

13. Ness, N. F., C. S. Scearce, and J. B. Seek, Initial results of the IMP-1 magnetic field experiment, J. Geophys. Res. 69, 3531-3569, 1964.

14. Sonett, C. P., E. J. Smith and A. R. Sims, Surveys of the distant geomagnetic field: Pioneer I and Explorer VI, Space Research I, 921, 1960. 
15. Sonett, C. P. and others, The distant geomagnetic field, parts I to V, J. Geophys. Res. 67 and 68.

16. Sonett, C. P., D. S. Colburn, L. Davis, Jr., E. J. Smith, and P. J. Coleman, Jr., Evidence for a collision-free magnetohydrodynamic shock in interplanetary space, Phys. Rev. Letters 13, 153-156, 1964.

17. Chapman, S. and V. C. A. Ferraro, A new theory of magnetic storms, Terrestrial Magnetism and Atmospheric Elec. 36, 77-97, 171-186, 1931; 37, 147-156, 421-429, $1932 ; 38,79-96,1933$.

18. Cahill, L. J. and P. J. Amazeen, The boundary of the geomagnetic field, J. Geophys. Res. $68,1835-43,1963$.

19. Johnson, F. S., The gross character of the geomagnetic field in the solar wind, $J$. Geophys. Res. 65, 3049, 1960.

20. Beard, D. B., The interaction of the terrestrial magnetic field with the solar corpuscular radiation, J. Geophys. Res. 65, 3559-3568, 1960.

21. Midgley, J. E. and Leverett Davis, Jr., Calculation by a moment technique of the perturbation of the geomagnetic field by the solar wind, J. Geophys. Res. 68, 51115123, 1963.

22. Mead, G. D. and D. B. Beard, Shape of the geomagnetic field solar wind boundary, J. Geophys. Res. 69, 1169-1179, 1964.

23. Mead, G. D., Deformation of the geomagnetic field by the solar wind, J. Geophys. Res. 69, 1181-1195, 1964.

24. Dungey, J. W., Interplanetary magnetic field and the auroral zones, Phys. Rev. Letters $6,47,1961$.

25. Axford, W. I., H. E. Petschek, and G. L. Siscoe, The tail of the magnetosphere, $J$. Geophys. Res, to be published March 1, 1965.

26. Ness, N. F., The earth's magnetic tail, J. Geophys. Res. to be published.

27. Dessler, A. J. and R. D. Juday, Configuration of auroral radiation in space, to be published in Planetary and Space Science.

28. Dessler, A. J., Length of magnetospheric tail, J. Geophys. Res. 69, (19), 3913-3918, 1964.

29. Beard, D. B., The effect of an interplanetary magnetic field on the solar wind, $J$. Geophys. Res. 69, (7), 1159-1168, 1964.

30. Axford, W. I., and C. O. Hines, Unifying theory of high latitude geophysical phenomena and geomagnetic storms, Can. J. Phys., 39, 1433-1464, 1961.

31. Spreiter, J. R., and W. P. Jones, On the effect of a weak interplanetary magnetic field on the interaction between the solar wind and the geomagnetic field, J.G.R., 68, 3555-3565, 1963.

32. Heppner, J. P., N. F. Ness, T. L. Skillman, and C. S. Scearce, Explorer 10 magnetic field measurements, J.G.R., 68, 1-46, 1963. 
33. Freeman, J. W., Jr., The morphology of the electron distribution in the outer radiation zone and near the magnetospheric boundary as observed by Explorer 12, J.G.R. $69,1691-1724,1964$.

34. Serbu, G. P., Results from the IMP-1 retarding potential analyzer, Goddard Space Flight Center Report X-615-64-109, May, 1964, to be published in Space Research V, 1965.

35. Fan, C. Y., G. Gloeckler, and J. A. Simpson, Evidence for $>30 \mathrm{Kev}$ electrons accelerated in the shock transition region beyond the earth's magnetospheric boundary, Phys. Rev. Letters, 13 (5), 149-153, 1964.

36. Anderson, K. A., H. K. Harris, and R. J. Paoli, Energetic electron fluxes in and beyond the earth's outer magnetosphere, to be published.

37. Jokipii, J. R., and L. Davis, Jr., Acceleration of electrons near the earth's bow shock, Phys. Rev. Letters, 13 (25), 739-741, 1964.

37a. Ness, N. F., The magnetohydrodynamic wake of the moon, J. Geophys. Res. 70 (3), February 1, 1965.

38. Van Allen, J. A., First public lecture on the Discovery of the geomagnetically trapped radiation, State Univ. of Iowa Report SUI 60-13, 1960.

39. Störmer, C., The Polar Aurora, Clarendon Press, Oxford, 1955.

40. Singer, S. F., A new model of magnetic storms, Trans. A.G.U., 38, 175, 1957.

41. Christofilos, N. C., The Argus experiment, J.G.R., 64, 869, 1959.

42. McHwain, C. E., Coordinates for mapping the distribution of magnetically trapped particles, J.G.R., 66, 3681, 1961.

43. Vernov, S. N., and A. E. Chudakov, Terrestrial corpuscular radiation and cosmic rays, in Space Research $I$, p. 751, 1960.

44. Van Allen, J. A., C. E. McIlwain and G. H. Ludwig, Radiation observations with Satellite $1958 \epsilon$, J.G.R., 64, 271, 1959

45. Van Allen, J. A. and L. A. Frank, Radiation around the earth to a radial distance of $107,400 \mathrm{~km}$, Nature, 183, 430, 1959.

46. Van Allen, J. A. and L. A. Frank, Radiation measurements to $658,300 \mathrm{~km}$ with Pioneer IV, Nature, 184, 219, 1959.

47. Freden, S. C. and R. S. White, Protons in the earth's magnetic field, Phys. Rev. Letters 3, 9, 1959.

48. Freden, S. C. and R. S. White, Trapped protons and cosmic ray albedo neutron fluxes, JGR 67, 25, 1962.

49. Armstrong, A. H. and F. B. Harrison, Charged particles in the inner Van Allen radiation belt, JGR $66,351,1961$.

50. Heckman, H. and A. H. Armstrong, Energy spectrum of geomagnetically trapped protons, JGR 67, 1255, 1962. 
51. Kellogg, P. J., Possible explanation of the radiation observed by Van Allen at high altitudes in satellites, Il Nuovo Cimento $11,48,1959$.

52. Singer, S. F., Trapped albedo theory of the radiation belt, Phys. Rev. Let. 1, 181, 1958.

53. Freden, S. C. and R. S. White, Particle fluxes in the inner radiation belt, JGR 65, 1377,1960 .

54. Blanchard, R. C. and W. N. Hess, Solar cycle changes in inner zone protons, JGR $69,3927,1964$.

55. Pizzella, G., C. E. McIlwain and J. A. Van Allen, Time variations of intensity in the earth's inner radiation zone October 1959 through December 1960, JGR 67, 1235, 1962.

56. Naugle, J. E. and D. A. Kniffen, Variations of the proton energy (1963) spectrum with position in the inner Van Allen belt, JGR 67, 4065.

57. Lenchek, A. M., On the anomalous component of low-energy geomagnetically trapped protons, JGR 67, 2145, 1962.

58. McIlwain, C. E., The radiation belts, natural and artificial, Science 142, 355-361, 1963.

59. McIlwain, C. E., Redistribution of trapped protons during a magnetic storm, Space Research $V$, to be published.

60. Holly, F. E., 1. Allen and R. G. Johnson, Radiation measurements to 1500 kilometers altitude at equatorial latitudes, JGR 66, 1627, 1961.

61. Freeman, J. W., Detection of an intense flux of low energy protons or ions trapped in the inner radiation zone, JGR 67, 921, 1962.

62. Hess, W. N., The effects of high-altitude explosions, in Space Physics, edited by Donald P. LeGalley and Alan Rosen, 573-610, 1964.

63. Van Allen, J. A., C. E. McIlwain and G. H. Ludwig, Satellite observations of electrons artificially injected into the geomagnetic field, JGR 64, 877, 1959.

64. O'Brien, B. J., C. D. Laughlin and J. A. Van Allen, Geomagnetically trapped radiation by a high altitude nuclear explosion on July 9, 1962, Nature 195, 939, 1962.

65. Pieper, G. F., D. J. Williams and L. A. Frank, TRAAC observations of the artificial radiation belt from the July 9, 1962 nuclear detonation, JGR 68,635, 1963.

66. Brown, W. L. and J. D. Gabbe, The electron distribution in the earth's radiation belts during July, 1962 as measured by Telstar, JGR 68,607, 1963.

67. Durney, A. C., H. Elliot, R. J. Hynes and J. J. Quenby, Satellite observations of the energetic particle flux produced by the high altitude nuclear explosions of July 9, 1962, Nature 195, 1245, 1962.

68. Galperin, I. and A. D. Bolivnova, The study of the drastic changes of the radiation in the upper atmosphere in July, 1962, in Space Research $V$, to be published. 
86. McDiarmid, I. B. and J. R. Burrows, Diurnal intensity variations in the outer radiation zone at $1000 \mathrm{~km}$, Can. J. Phys. 42, 1135, 1964.

87. Williams, D. W. and W. F. Palmer, Distortions in the radiation cavity as measured by an $1100 \mathrm{~km}$ polar orbiting satellite, J. Geophys. Res. to be published February, 1965.

88. Sloanaker, R. M., Apparent temperature of Jupiter at a wave length of $10 \mathrm{~cm}$, Astronomical J. 64, 346, 1959.

89. Drake, F. D. and H. Hvatum, Non-thermal microwave radiation from Jupiter, A. J. 64, $329,1959$.

90. Radhakrishman, V., and J. A. Roberts, Polarization and angular extent of the $\mathbf{9 6 0}$ Mc/s radiation from Jupiter, Phys. Rev. Let. 4, 493, 1960.

91. Chang, D. B. and L. Davis, Synchrotron radiation as the source of Jupiter's polarized decimeter radiation, $A p . J .136,657,1962$.

92. Anderson, H. R., Energetic particles measured near Venus by Mariner 2, J. Geo. Res. 69, 2651, 1964.

93. Van Allen, J. A. and L. A. Frank, The mission of Mariner 2 preliminary observations: the Iowa radiation experiment, Science 138, 1097, 1962.

94. Dolginov, S. Sh., E. G. Evoshenko, L. N. Zhuzgov, N. V. Pushkov and L. O. Tyurmina, Measuring the magnetic fields of the earth and moon by means of Sputnik III and Space Rockets I and II, Space Research I, 11, 1960.

95. Gringaus, K. I., Some results of experiments in interplanetary space by means of charged particle traps on Soviet Space probes, Space Research I, edited by H. C. van de Hulst, C. deJager and A. F. Moore, North-Holland Publishing Co., Amsterdam, 1961.

96. Frank, L. A., J. A. Van Allen, and H. K. Hills, Mariner 2: preliminary reports on measurements of Venus; charged particles, Science 139, 905-910, March 8, 1963.

97. Gringaus, K. I., V. G. Kurt, V. I. Morog, and I. C. Sklovskiy, Sbornik 'Iskusstvennye Sputniki Zemli' Izd. AN SSSR, 6, 108, 1961.

98. O'Brien, B. J., J. A. Van Allen, C. D. Laughlin, and L. A. Frank, Absolute electron intensities in the heart of the earth's outer radiation zone, J. Geophys. Res. 67, $397-403,1962$.

99. Mcllwain, C. E., Direct measurements of particles producing visible auroras, $J$. Geophys. Res. 65, 2727-2747, 1960.

100. Davis, L. R., O. E. Berg, and L. H. Meredith, Direct measurements of particle fluxes in and near auroras, Space Research, edited by H. K. Kalmen-Bijl, NorthHolland Publishing Co., Amsterdam, 721-735, 1960.

101. McDiarmid, I. B., D. C. Rose, and E. Budzinski, Direct measurements of charged particles associated with auroral zone radio absorption, Can. J. Phys. 39, 1888$1900,1961$. 
69. West. H. I., L. G. Mann and S. D. Bloom, The electron spectra in the radiation belts in the fall of 1962, in Space Research $V$, to be published.

69a. Van Allen, J. A., L. A. Frank, and B. J. O'Brien, Satellite observations of the artificial radiation belt of July 1962, J. Geophys. Res. 68, 619-627, 1963.

70. McDiarmid, I., J. Burrow, E. Budzinski and D. Rose, Satellite measurements in the starfish artificial radiation zone, Can. J. Phys. 41, 1332, 1963.

71. Welch, J. A., R. L. Kaufmann and W. N. Hess, Trapped electron time histories for $\mathrm{L}=1.18$ to $\mathrm{L}=1.30, J$. Geophys. Res. $68,685,1963$.

72. Walt, M., The effects of atmospheric collisions on geomagnetically trapped electrons, J. Geophys. Res. 69, 3997, 1964.

73. Dungey, J. W., Loss of Van Allen electrons due to whistlers, Planet. Spa. Science 11, $591,1963$.

74. Filz, R., H. Yagoda and E. Holeman, Observations on trapped protons in emulsions recovered from satellite orbits, Space Research IV, 642, 1963.

75. Davis, L. R. and J. M. Williamson, Low energy trapped protons, in Space Research III, 365, 1963.

76. Jensen, D. C. and J. C. Cain, An interim geomagnetic field, abstract, JGR 67, 3568, 1962.

77. Nakada, M. P., J. W. Dungey and W. N. Hess, Theoretical studies of protons in the outer radiation belts, in Space Research $V$, to be published.

78. Nakada, M. P., G. D. Mead, The shape of the outer belt protons, JGR to be published.

79. Hoffman, R. A., P. A. Bracken, Magnetic effects of the quiet time proton belt, GSFC document X-611-64-186.

80. Cladis, J. B., L. F. Chase, W. L. Imhof and D. J. Knecht, Energy spectrum and angular distributions of electrons trapped in the geomagnetic field, JGR 66,2297 , 1961.

81. Forbush, S. E., G. Pizzella and D. Venkateson, The morphology and temporal variations of the Van Allen radiation belt October, 1959 to December, 1960, JGR 67, $3651,1962$.

82. O'Brien, B. J., Lifetimes of outer zone electrons and their precipitation into the atmosphere, JGR 67, 3687, 1962.

83. O'Brien, B. J., High latitude geophysical studies with Injun 3 (3) precipitation of electrons into the atmosphere, J.Geo.Res. 69, 13, 1964.

84. Frank, L. A., J. A. Van Allen, and E. Macagno, Charged particle observations in the earth's outer magnetosphere, J. Geo. Res. 68, 3543, 1963.

85. O'Brien, B. J., A large diurnal variation of the geomagnetically trapped radiation, J. Geo. Res. 68, 989, 1963. 
102. Mann, L. G., S. D. Bloom, and H. I. West, Jr., The electron spectrum from 90 to $1200 \mathrm{kev}$ as observed on Discoverer satellites 29 and 31, Space Research III, edited by W. Priester, North-Holland Publishing Co., Amsterdam, 447-462, 1963.

102a. Brown, R. R., Balloon observations of auroral zone x-rays, Proc. of the International Conference on Cosmic Rays and the Earth Storm, Kyoto, Sept. 4-15, 1961, I. Earth Storm, 236, 1962.

102b. Anderson, K. A., Balloon observations of $x$-rays in the auroral zone $I$ and II, $J$. Geophysical Research 65, 551 and 65, 521, 1960.

103. Anderson, K. A. and D. W. Milton, Balloon observations of $\mathrm{x}$-rays in the auroral zone, 3 High time resolution studies, J. Geophys. Res. 69, 4457-4479, 1964.

104. Sharp, R. D., J. E. Evans, W. L. Imhof, R. G. Johnson, J. B. Regan and R. V. Smith, Satellite measurements of low energy electrons in the northern auroral zone, $J$. Geophys. Res. 69, 2721, 1964.

105. Gurnett, D. A. and B. J. O'Brien, High latitude geophysical studies with satellite Injun 3,5 . Very low frequency electromagnetic radiation, J. Geophys. Res. 69, $65-90,1964$.

106. O'Brien, B. J., and H. Taylor, High latitude geophysical studies with satellite Injun 3, 5. Auroras and their excitation, J. Geophys. Res. 69, 45-64, 1964.

107. Cain, J. C., I. R. Shapiro, J. D. Stolarik, and J. P. Heppner, Vanguard 3 magnetic field observations, J. Geophys. Res. 67, (13), 5055-5069, 1962.

108. Heppner, J. P., The world magnetic survey, Space Science Revs, 2, 315-354, 1963.

109. Cahill, L. J., Jr., Preliminary results of magnetic field measurements in the tail of the geomagnetic cavity, I. G. Bulletin No. 79, Trans, AGU 45, 231-235, 1964.

110. Akasofu, S. I., The main phase of magnetic storms and the ring current, Space Sci. Revs. 2, 91-135, 1963.

111. Smith, E. J., Theoretical and experimental aspects of ring current, in Space Science, ed. Le Galley, 316-373, 1963.

112. Smith, E. J., C. P. Sonett, and J. W. Dungey, Satellite observations of the geomagnetic field during magnetic storms, J. Geophys. Res. 69, 2669-2688, 1964.

113. Nishida, A. and L. J. Cahill, Jr., Sudden impulses in the magnetosphere observed by Explorer 12, J. Geophys. Res. 69, 2243-2255, 1964.

114. Forbush, S. E., Three unusual cosmic-ray increases possibly due to charged particles from the sun, Phys. Rev. 70, 771-772, 1946.

115. Meyer, P., E. N. Parker, and J. A. Simpson, Solar cosmic rays of February 1956 and their propagation through interplanetary space, Phys. Rev. 104, (3), 768$783,1956$.

116. Winckler, J. R., The production and propagation of energetic particles from the sun, in Space Science, ed. Le Galley, 427-484, 1963. 
117. Charakhchyan, A. N., V. F. Tulinov and T. N. Charakhchyan, Cosmic rays emitted by the sun, Proc. of the First International Space Science Symposium, Nice, January $11-16,1960,649-661,1960$.

118. Winckler, J. R., Geomagnetic and interplanetary effects on solar cosmic rays, Proc. of the International Conference on Cosmic Rays and the Earth Storm, Kyoto, September 4-15. 1961, II. Joint Sessions, 353-360, 1962; and also J. Phys. Soc. of Japan 17, Supplement A-II, 353, 1962.

119. Fichtel, C. E. and D. E. Guss, Heavy nuclei in solar cosmic rays, Phys. Rev. Letters 6, 495, 1961.

120. Lin, W. C., Observation of galactic and solar cosmic rays from October 13, 1959 to February 17, 1961 with Explorer VII, State Univ. Iowa Rept. 61-16, 1961.

121. Pieper, G. F., A. J. Zmuda, C. A. Bostrom and B. J. O'Brien, Solar protons and magnetic storms in July 1961, J. Geophys. Res. 67, 4959, 1962.

122. McDonald, F. B., editor, Solar proton manual, NASA TR R-169, December, 1963.

123. Bryant, D. A., T. L. Cline, U. D. Desai, and F. B. McDonald, Explorer XII observations of solar cosmic rays and energetic storm particles after the solar flare of September 28, 1961, J. Geophys. Res. 67, 4983-5000, 1962.

124. Bryant, D. A., T. L. Cline, U. D. Desai and F. B. McDonald, Studies of solar protons with Explorers XII and XIV, GSFC X-611-64-217, July, 1964.

125. McCracken, K. G., The cosmic-ray flare effect. I. Some new methods of analysis, J. Geophys. Res. 67 (2), 423-434, 1962.

126. Bryant, D. A., T. L. Cline, U. D. Desai, F. B. McDonald, New evidence for longlived solar streams in interplanetary space, Phys. Rev. Letters 11, (4), 144-146, 1963.

127. Parker, E. N., Acceleration of cosmic rays in solar flares, Phys. Rev. 107, (3), 830-836, 1957.

128. Petschek, H. E., Magnetic field annihilation, AAS-NASA Symposium on the Physics of Solar Flares: Proc. Symp. Held at GSFC, Greenbelt, Md. Oct. 28-30, 1963, edited by W. N. Hess, NASA SP-50, 425-439, 1964.

129. Severny, A. B., Generation of flares during the change of solar magnetic fields, Astronomicheskiy Zhurnal 38, 403-408, 1961 (in Russian).

130. Hess, W. N., editor, AAS-NASA Symposium on the Physics of Solar Flares, NASA SP-50, 1964.

131. Frier, P., W. R. Webber, Radiation hazard in space from solar particles, Science $142,1587,1963$.

132. Anderson, K. A., Preliminary study of prediction aspects of solar cosmic ray events, NASA TN D-700, April 1961.

133. Waddington, C. J., Composition of the primary cosmic radiation, Progress in Nuclear Physics, 8, 1, 1960. 
134. Earl, J. A, Cloud-chamber observations of primary cosmic ray electrons, Phys. Rev. Letters, 6, No. 3, 125-128, 1961.

135. Meyer, P., and R. Vogt, Electrons in the primary cosmic radiation, Phys. Rev. Letters, 6, No. 4, 193-196, 1961.

136. Cline, T. L., G. H. Ludwig, and F. B. McDonald, Detection of interplanetary 3- to 12-MeV electrons, Phys. Rev. Letters, 13, No. 26, 786-789, 1964.

137. DeShong, J. A., R. H. Hildebrand, and P. Meyer, Ratio of electrons to positrons in the primary cosmic radiation, Phys. Rev. Letters, 12, 3, 1964.

138. Hayakawa, S., and H. Okuda, Electronic components in galactic cosmic rays, Progr. Theor. Phys., 28, 517-528, 1962.

139. McDonald, F. B., and G. H. Ludwig, Measurement of low-energy primary cosmicray protons on IMP-1 satellite, Phys. Rev. Letters, 13, No. 26, 783-785, 1964.

140. Fan, C. Y., P. Meyer, and J. A. Simpson, The rapid reduction of cosmic radiation intensity measured in interplanetary space, Phys. Rev. Letters, 5, 43, 1960.

141. Hess, W. N., and A. J. Starnes, Measurement of the neutron flux in space, Phys. Rev. Letters, 5, 48-50, 1960.

142. Trainor, J. H., and J. A. Lockwood, Neutron albedo measurements on polar orbiting satellites, J. Geophys. Res., 69 3115-3123, 1964.

143. Martin, J. P., L. Witten and L. Katz, Measurement of cosmic ray albedo neutron flux above the atmosphere, J. Geophys. Res., 68, 2613-2618, 1963.

144. Williams, D. J., and C. O. Bostrom, Albedo neutrons in space, J. Geophys. Res., $69,377-391,1964$.

145. Hess, W. N., H. W. Patterson, R. Wallace, and E. L. Chupp, Cosmic-ray neutron energy spectrum, Phys. Rev., 116, 445-457, 1959.

146. Lingenfelter, R. E., The cosmic-ray neutron leakage flux, J. Geophys. Res., 68, $5633,1963$.

147. Lingenfelter, R. E., and E. J. Flamm, Neutron leakage flux from interactions of solar protons in the atmosphere, J. Geophys. Res., 69, 2199-2207, 1964.

148. Hess, W. N., Neutrons in Space, NASA TN D-1696, February 1963. 